NUREG/CR-5721 SAND91-0947

\title{
Video Systems for Alarm Assessment
}

Rorined by

OCT 221991

Prepared by

D. A. Greenwoll, J. C. Matter/SNL

P. E. Ebel/BEI

Sandia National Laboratories

BE Inc.

Prepared for

U.S. Nuclear Regulatory Commission 


\section{AVAILABILITY NOTICE}

Availability of Reference Materials Cited in NRC Publications

Most documents clted in NRC publications will be avallable from one of the following sources:

1. The NRC Public Document Room, 2120 L Street. NW., Lower Level, Washington, DC 20555

2. The Superintendent of Documents, U.S. Government Printing Office, P.O. Box 37082, Washington, DC 20013-7082

3. The National Technical Information Service, Springfleld, VA 22161

Although the listing that follows represents the majority of documents clted In NRC publications, it is not intended to be exhaustive.

Referenced documents avallable for inspection and copying for a fee from the NRC Public Document Room include NRC correspondence and internal NRC memoranda; NRC bulletins, circulars, information notices, Inspection and Investigation notices; lisensee event reports; vendor reports and correspondence; Commisslon papers; and applicant and licensee documents and correspondence.

The following documents in the NUREG series are avallable for purchase from the GPO Sales Program: formal NRC staff and contractor reports, NRC-sponsored conference proceedings, international agreement reports, grant publications, and NRC booklets and brochures. Also avallable are regulatory guldes, NRC regulations in the Code of Federal Regulations, and Nuclear Regulatory Commission Issuances.

Documents avallable from the National Technical Information Service Include NUREG-serles reports and technical reports prepared by other Federal agencles and reports prepared by the Atomic Energy Commission, forerunner agency to the Nuclear Regulatory Commission.

Documents avallable from public and special technical libraries include all open literature items, such as books, Journal articles, and transactions. Federal Register noticeo, Federal and State legislation, and congressional reports can usually be obtained from these libraries.

Documents such as theses, dissertations, forelgn reports and translations, and non-NRC conference proceedings are available for purchase from the organization sponsoring the publication clted.

Single coples of NRC draft reports are avallable free, to the extent of supply, upon written request to the Office of Administration, Distribution and Mall Services Section, U.S. Nuclear Regulatory Commission, Washington, DC 20555.

Coples of industry codes and standards used in a substantive manner in the NRC regulatory process are maintained at the NRC Library, 7920 Norfolk Avenue. Bethesda, Maryland, for use by the publlc. Codes and standards are usually copyrighted and may be purchased from the originating organization or, If they are American National Standards, from the American National Standards Institute, 1430 Broadway, New York, NY 10018.

\section{DISCLAIMER NOTICE}

This report was prepared as an account of work sponsored by an agency of the United States Government. Neither the United States Government nor any agency thereof, or any of their employees, makes any warranty, expressed or implied, or assumes any legal liability of responsibility for any third party's use, or the results of such use, of any information, apparatus, product or process disclosed in this report, or represents that its use by such third party would not infringe privately owned rights. 
NUREG/CR- -5721

TI92 001662

\section{Video Systems for Alarm Assessment}

Manuscript Completed: September 1991

Date Published: September 1991

Prepared by

D. A. Greenwoll, J. C. Matter, Sandia National Laboratories

P. E. Ebel, BE Inc.

Sandia National Laboratories

Albuquerque, NM 87185

Subcontractor:

BE Inc.

P.O. Box 381

Hwy. 278, Airport Industrial Park

Barnwell, SC 29812

\section{Prepared for}

Division of Safeguards and Transportation

Office of Nuclear Material Safety and Safeguards

U.S. Nuclear Regulatory Commission

Washingion, DC 20555

NRC FIN Li387

\section{DISCLAIMER}

This report was prepared as an account of work sponsored by an agency of the United States Government. Neither the United States Government nor any agency thereof, nor any of their employees, makes any warranty, express or implied, or assumes any legal liability or responsibility for the accuracy, completeness, or usefulness of any information, apparatus, product, or process disclosed, or represents that its use would not infringe privately owned rights. Reference herein to any specific commercial product, process, or service by trade name, trademark, manufacturer, or otherwise does not necessarily constitute or imply its endorsement, recommendation, or favoring by the United States Government or any agency thereof. The views and opinions of authors expressed herein do not necessarily state or reflect those of the United States Government or any agency thereof.

\section{MASTER}

DISTRIBUTION OF THIS DOCUMENT IS UNLIMITED

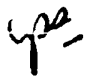




\begin{abstract}
The purpose of this NUREG is to present technical information that should be useful in designing video systems for alarm assessment. There is a section on each of the major components in a video system: camera, lens, lighting, transmission, synchronization, switcher, monitor, and recorder. Each section includes information on component selection, procurement, installation, test, and maintenance. Considerations for system integration of the components are contained in each section. System emphasis is focused on perimeter intrusion detection and assessment systems. A glossary of video terms is included.
\end{abstract}




\section{CONTENTS}

SECTION

Page

ABSTRACT ..iii

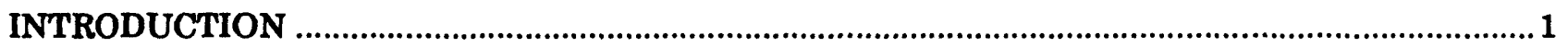

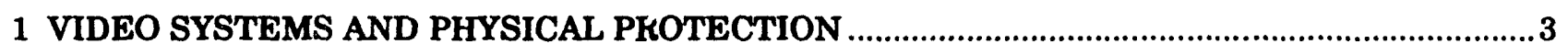

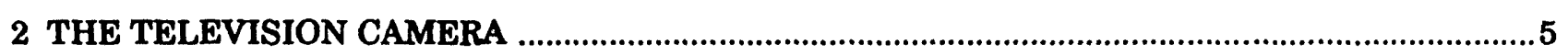

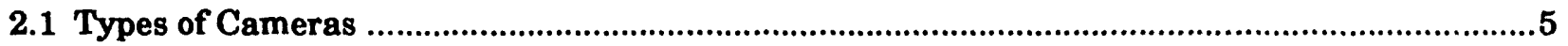

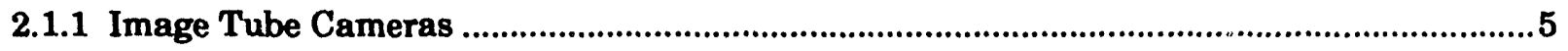

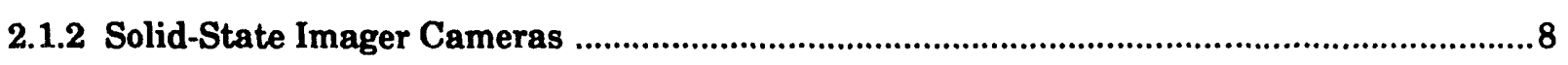

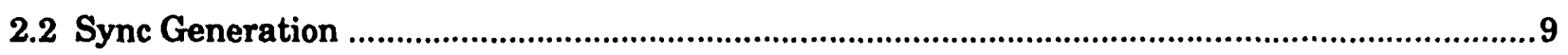

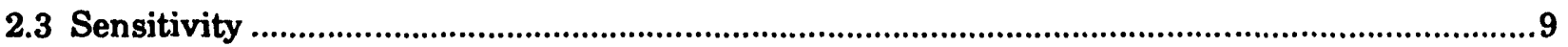

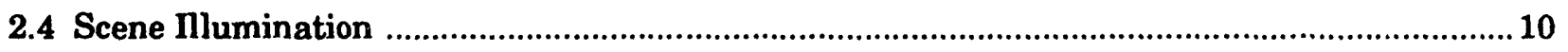

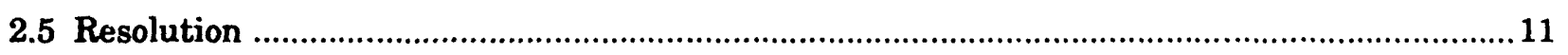

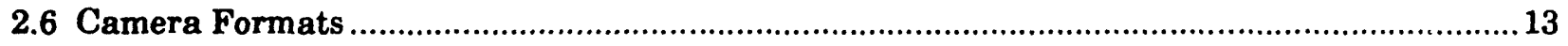

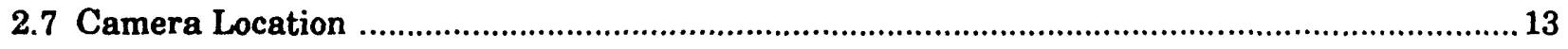

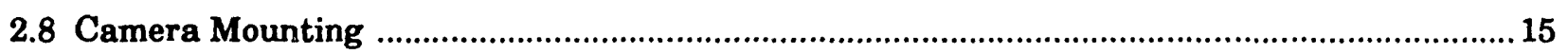

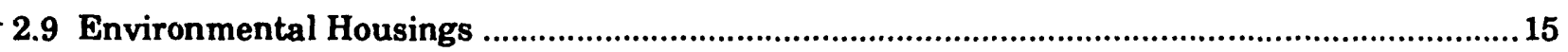

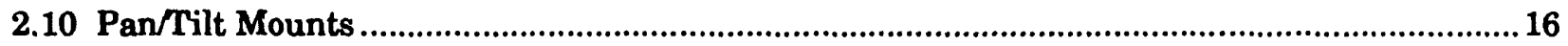

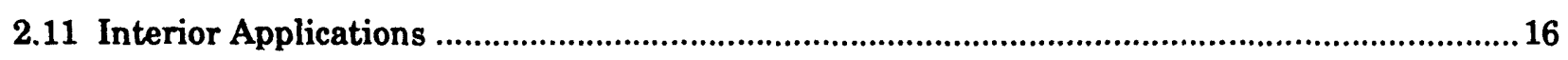

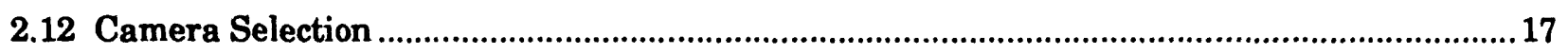

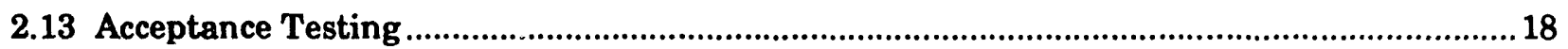

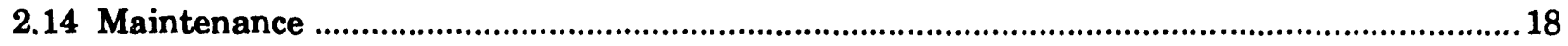

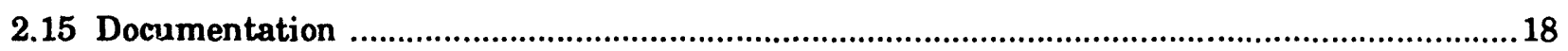

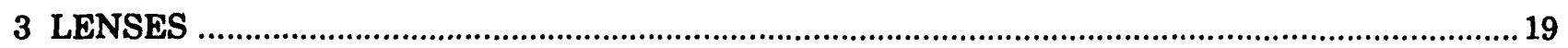

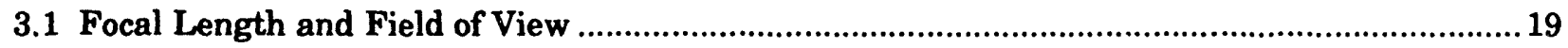

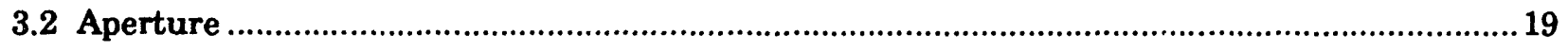

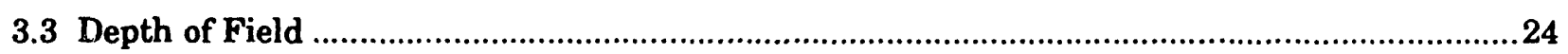

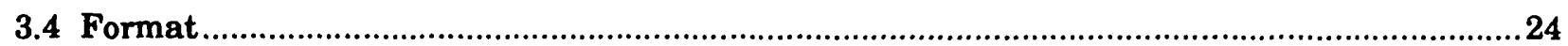

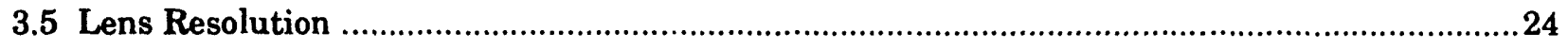

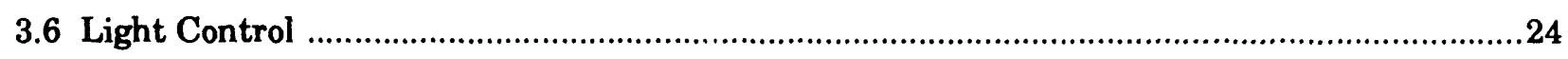

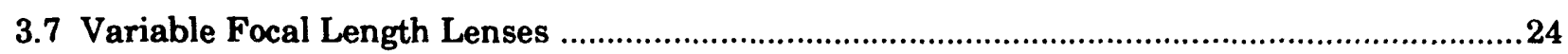

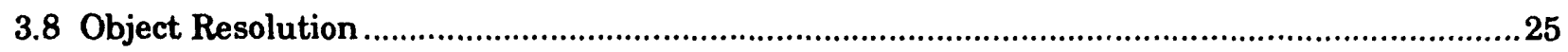

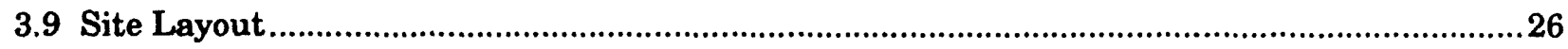

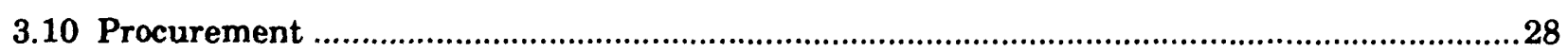

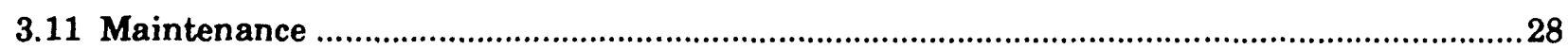

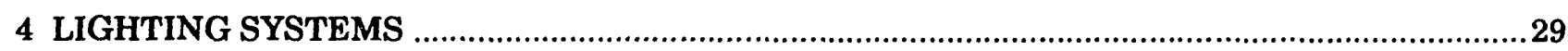

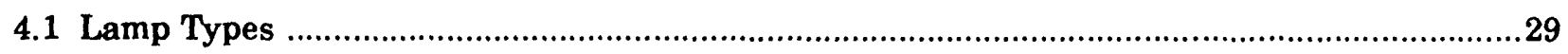




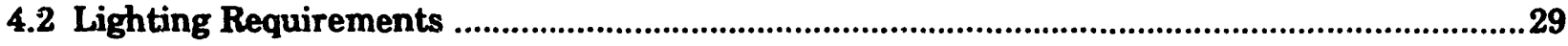

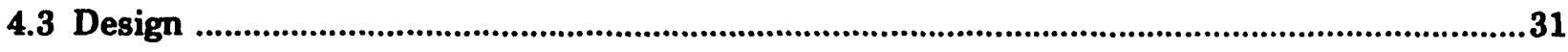

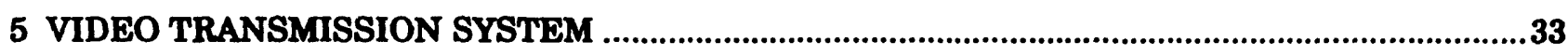

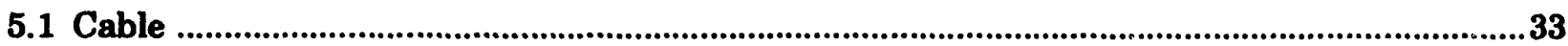

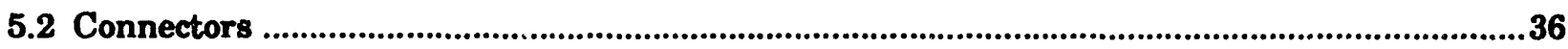

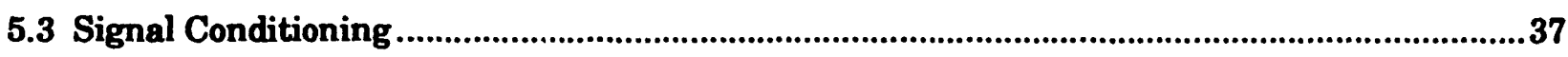

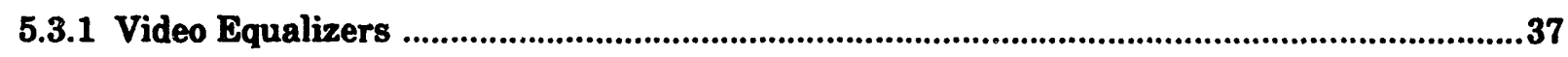

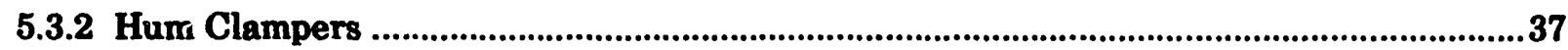

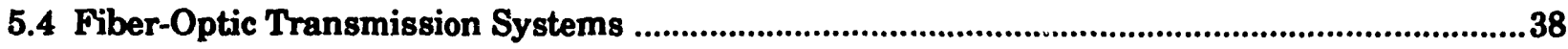

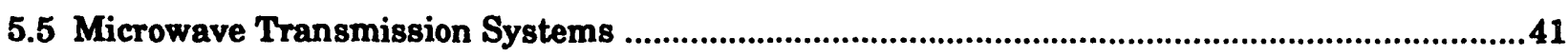

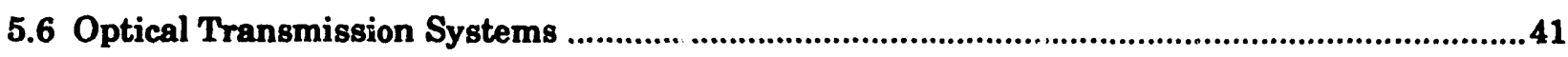

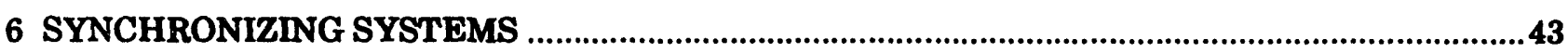

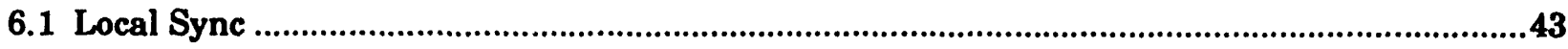

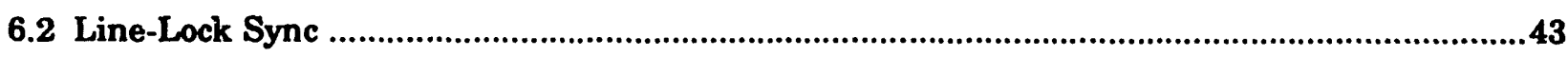

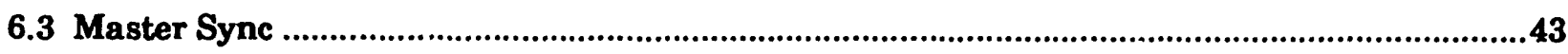

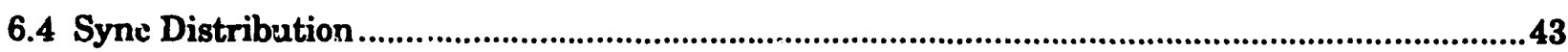

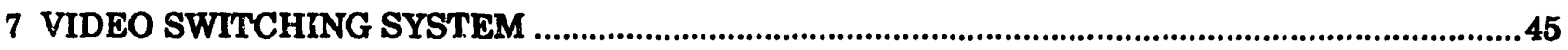

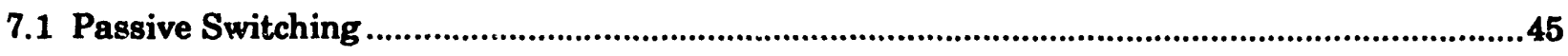

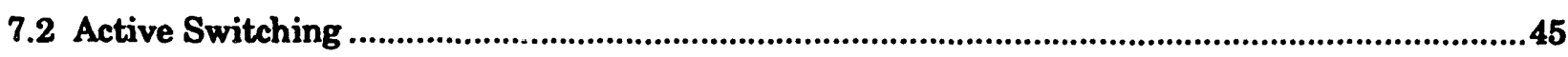

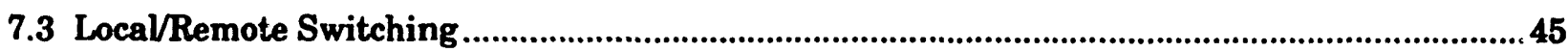

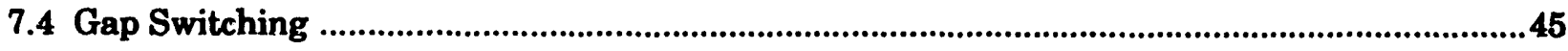

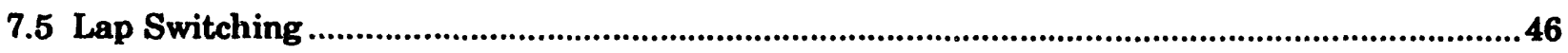

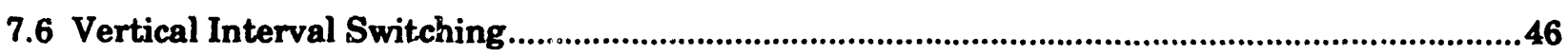

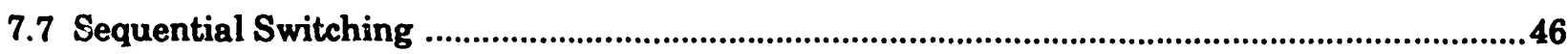

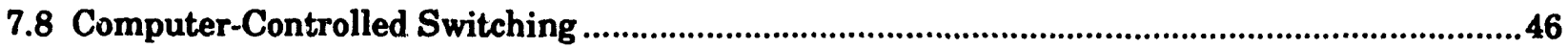

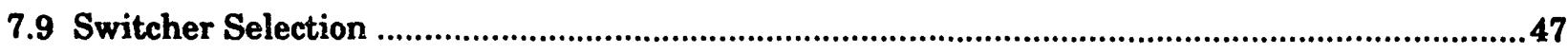

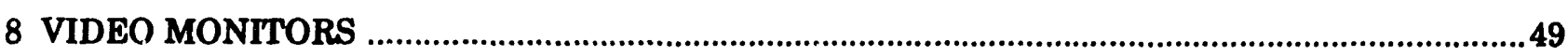

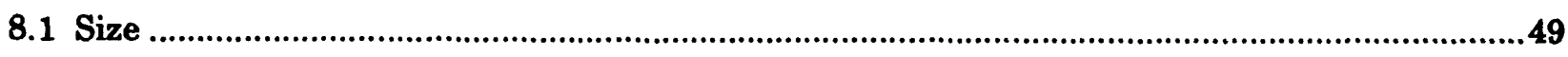

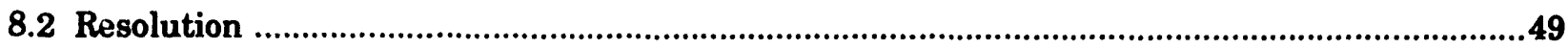

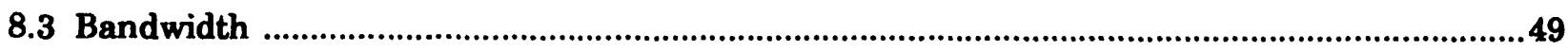

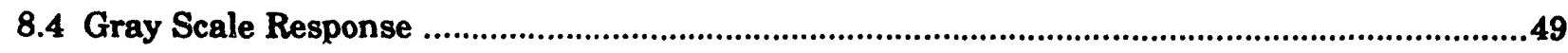

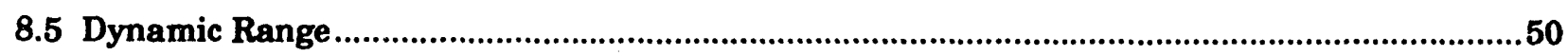

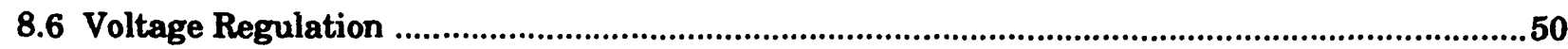

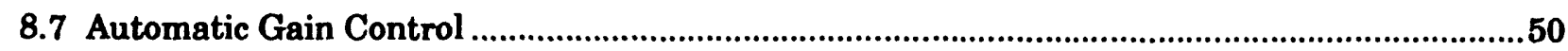

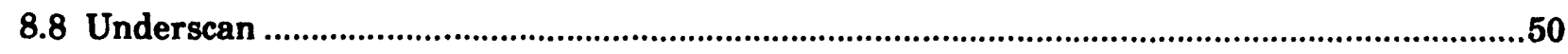

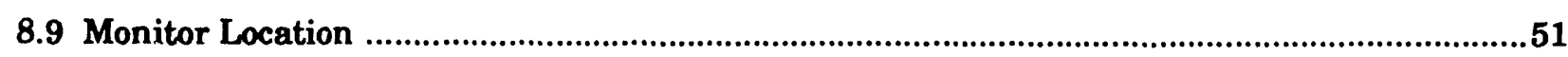

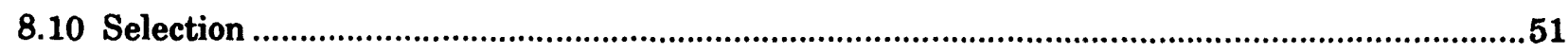

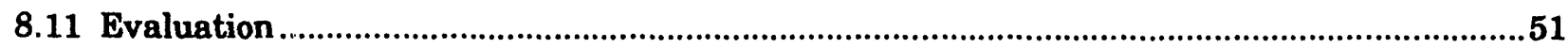




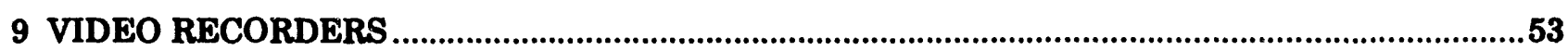

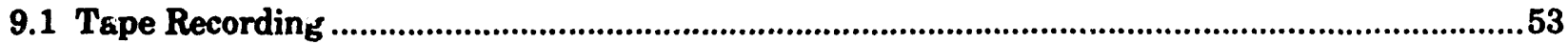

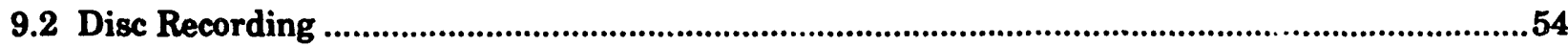

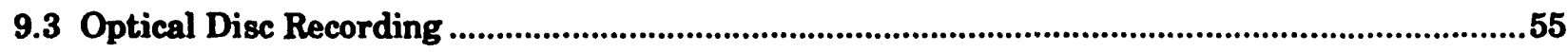

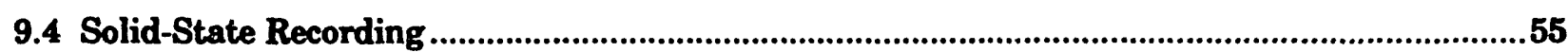

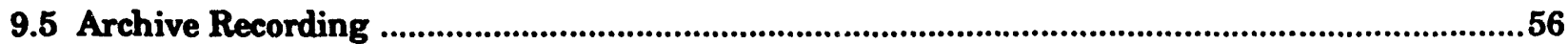

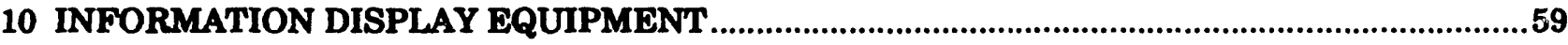

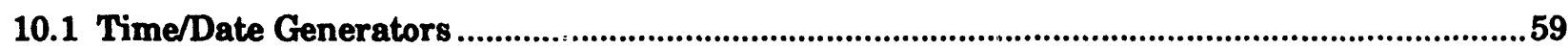

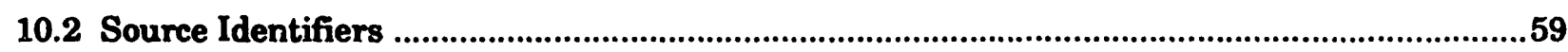

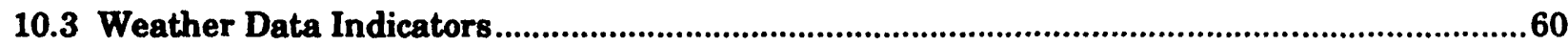

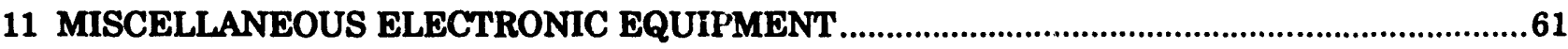

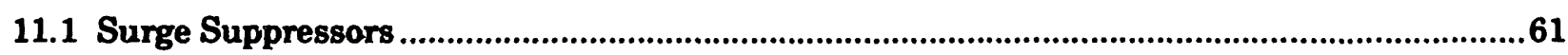

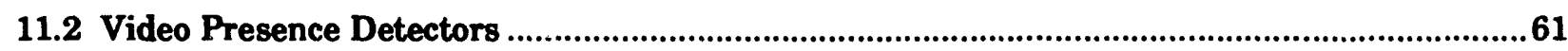

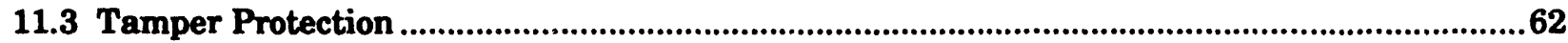

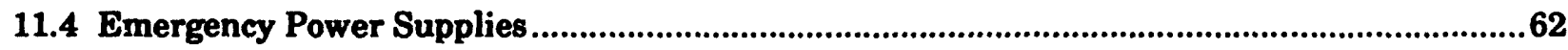

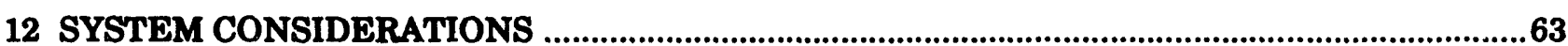

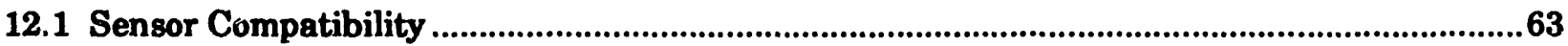

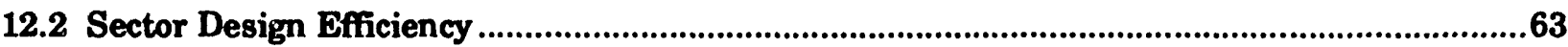

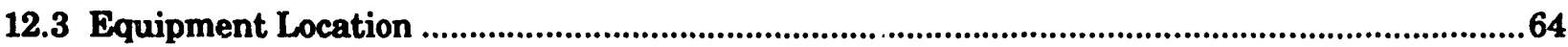

12.4 Maintenance

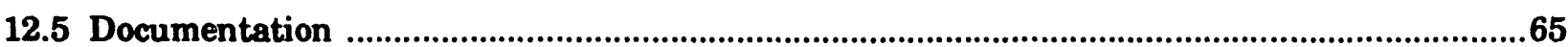

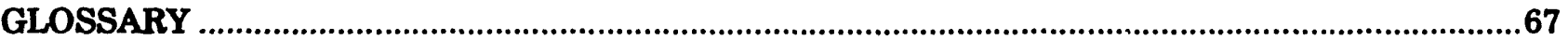




\section{FIGURES}

1-1. Block diagram of video assessment system ...............................................................................4

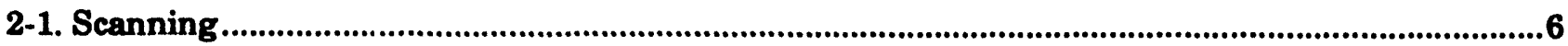

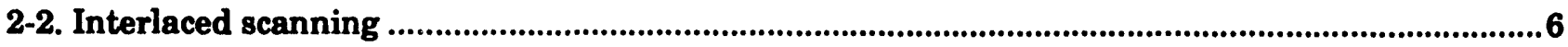

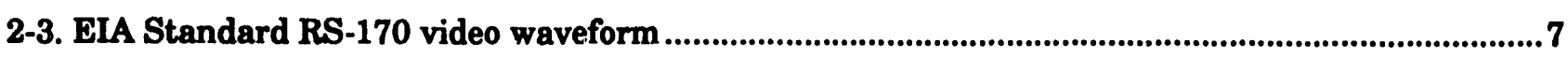

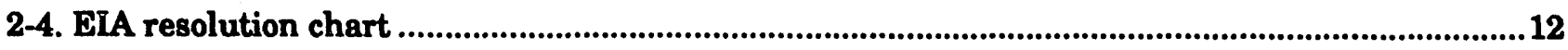

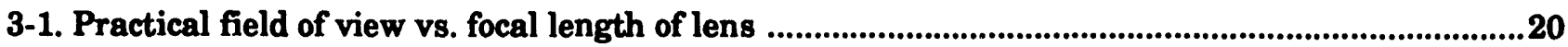

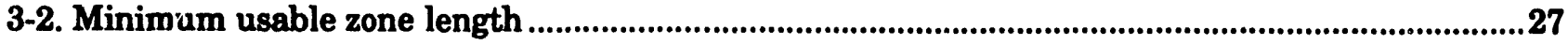

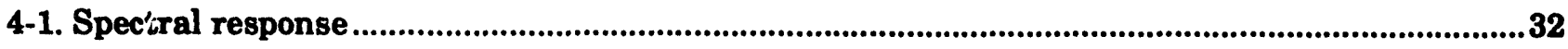

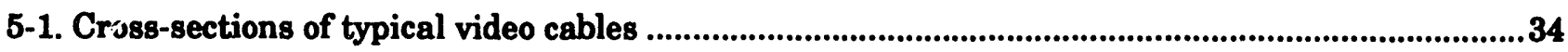

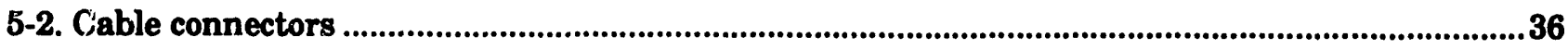

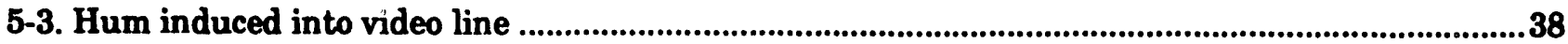

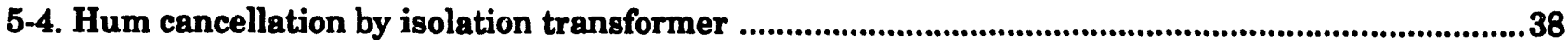

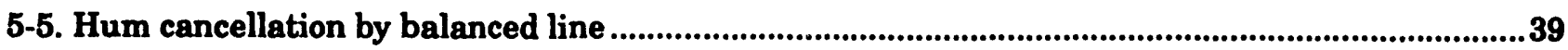




\section{TABLES}

2-1. Scene illumination levels .10

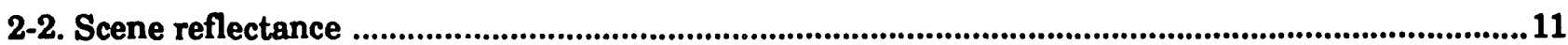

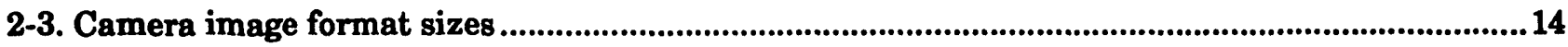

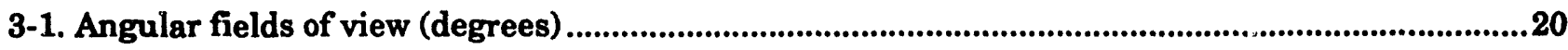

3-2. Horizontal linear fields of view for focal length from $16 \mathrm{~mm}$ (1-in.) camera format ......................21

3-3. Horizontal linear fields of view for focal length from $11 \mathrm{~mm}$ (2/3-in.) camera format.....................22

3-4. Horizontal lines:r fields of view for focal length from $8 \mathrm{~mm}$ (12-in.) camera format.......................23

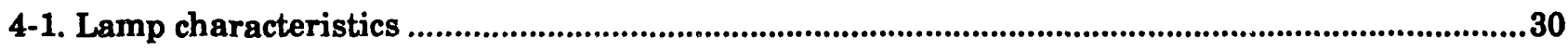

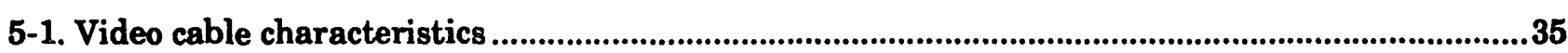




\section{INTRODUCTION}

U.S. Nuclear Regulatory Commission (NRC) regulations under Part 73 "Physical Protection of Plants and Material" of Title 10, Code of Federal Regulations, specify performance requirements for the physical protection of special nuclear materials and associated facilities. For fuel cycle facilities using or possessing a formula quantity of strategic special nuclear material, paragraph 73.45 íc)(1)(iii) calls for the use of detection and surveillance subsystems and procedures to discover and assess unauthorized activities and conditions and communicate them so that response can stop the activity or correct the conditions. For these facilities, an example reference system is outlined in paragraph 73.46. Paragraph 73.46(e)(8) states that all exterior areas within the protected area shall be monitored or periodically checked to detect the presence of unauthorized persons, vehicles, materials, or unauthorized activities.

Power reactor licensees are subject to the provisions of paragraph 73.55. Paragraph 73.55(h)(6) states "to facilitate initial response to detection of a penetration of the protected area and assessment of a threat, a capability of observing the isolation zones and the physical barrier at the perimeter of the protected area shall be provided, preferably by means of closed-circuit television or by other suitable means which limit exposure of responding personnel to attack." Detection of penetration or attempted penetration of the protected area or the isolation zone adjacent to the protected area must be sufficient to assure adequate response by the securiiy organization. Further, all exterior areas within the protected area must be periodically checked to detect the presence of unauthorized persons, vehicles, or material.

The purpose of this NUREG is to present technical information that may be of use in assembling a closedcircuit television (CCTV) system. By virtue of the nature of a NUREG document, the discussion of equipment or systems, herein, does not constitute acceptance or endorsement by the NRC. 


\section{VIDEO SYSTEMS AND PHYSICAL PROTECTION}

The basic functions of a physical protection system are (1) detection, (2) delay, and (3) response. Detection is the discovery of an adversary action. It includes sensing of covert or overt actions. The detection process is completed only when the sensor alarm is communicated to the security control center, and the system operator assesses the cause of the alarm. Delay is the slowing down of adversary progress. Delay can be accomplished by barriers and locks. Response consists of the actions taken by the protective force to prevent adversary success. It consists of interruption and containment of the threat. Interruption is defined as the response force arriving at the appropriate location to stop the adversary's progress. Containment of the threat is the act of stopping the adversary before his goal is accomplished. If any of these functions are missing or weak, it can degrade the performance of the entire system.

Detection is the first function an effective security system should perform. If detection capability is inadequate, then delay functions may not be effective, and response may never be called. Detection depends not only on sensors to detect an intrusion, but also on transmission and display of the alarm signal. In addition, the detection function relies on assessment to affirm quickly if the alarm is a real threat or a nuisance alarm.

A CCTV system can be used where assessment of a detection area is needed, especially where direct viewing is impossible, undesirable, or inconvenient. It not only can provide a better vantage point for assessment but also can augment the sensitivity, resolution, and spectral range of the human eye. In the absence of direct viewing, CCTV systems are a necessary part of the assessment system and should be designed as an element of a total physical protection system. There are numerous interactions among the video system, intrusion sensors, communications, and the display system that should be considered during conceptual design. To provide rapid assesement of large areas, CCTV systems are used to display, and frequently to record and playback, the alarm scene(s) at the controll center.

An optimum video assessment system would include the following:

- fixed cameras and lenses for each field of view of interest

- computer-controlled video switching equipment to automatically display an alarm scene on a normally blank video monitor

- solid-state video image capture devices to store and recall images when an alarm occurs

- monitors to observe additional concurrent alarm scenes and to accommodate manual selection of any camera scene

- equipment to manually initiate an archive of any camera scene

In assembling the optimum CCTV system, the designer first needs to be familiar with each component of the CCTV system, its relationship to other components in the CCTV system, and the various options, designs, and trade-offs that are available. In addition, the designer has to know the environmental constraints placed upon the system, the degree of resolution required, the financial constraints, and the projected maintenance requirements. This document is an attempt to cover all these factors in sufficient detail to allow the user independence from the manufacturer in the selection and maintenance of the CCTV system.

A basic video assessment system is composed of several cameras at remote points, a video display monitor at a central location, and various transmission and switching systems connecting the cameras to the monitor. The basic components, shown in Figure 1-1, include

- camera and lens (Sections 2 and 3)

- lighting system (Section 4)

- transmission system (Section 5)

- synchronization system (Section 6)
- video switching equipment (Section 7)

- video monitor (Section 8)

- video recording (Section 9) 


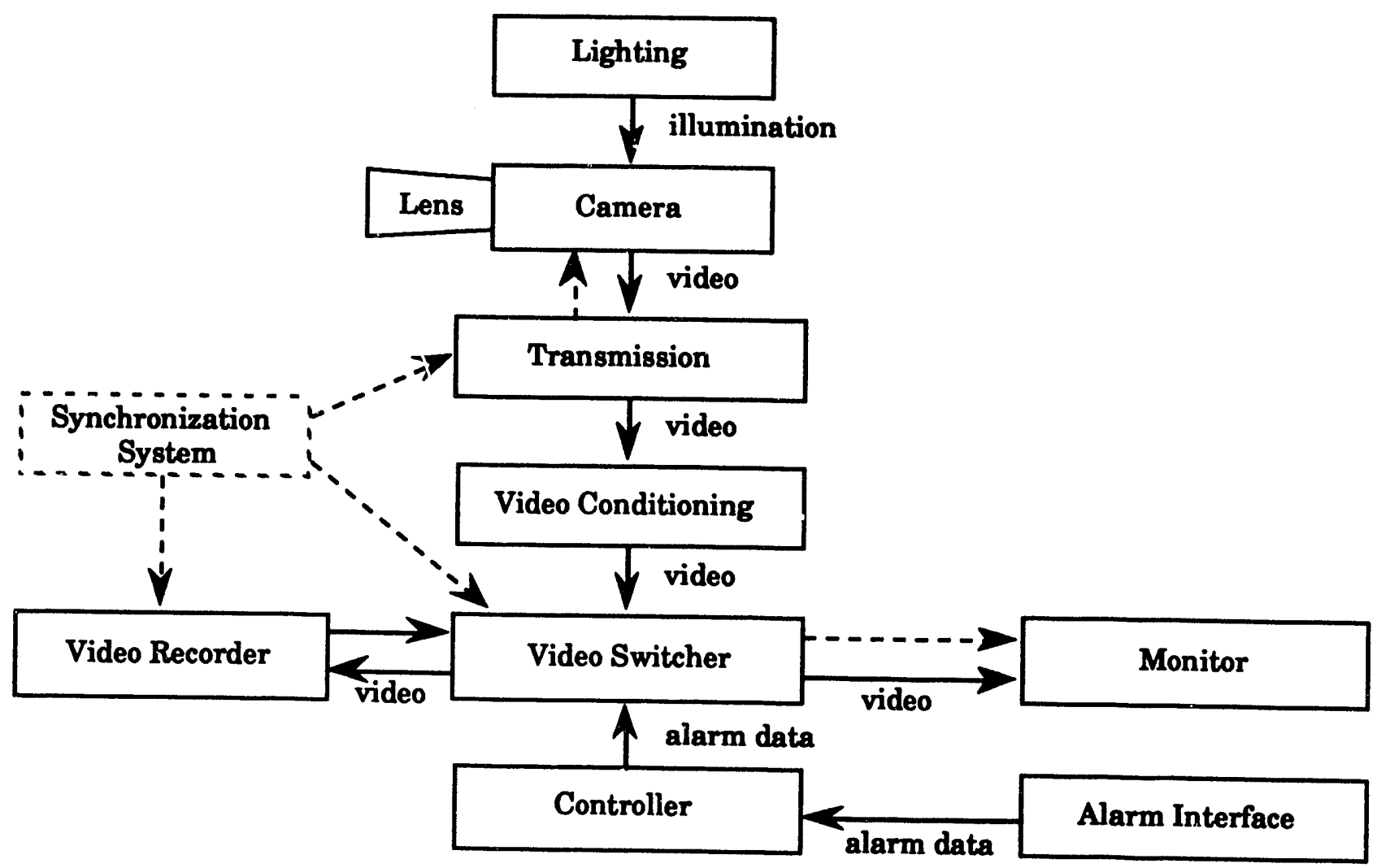

Figure 1-1. Block diagram of video assessment system 


\section{THE TELEVISION CAMERA}

A camera converts the optical image of the physical scene in the camera field of view into an electrical signal suitable for transmission over a variety of media to a video monitor for viewing by an observer. For a scene to be visible to the camera, it should be illuminated by sufficient natural or artificial light and should reflect a portion of that illumination into the camera lens. The light reaching the lens is then focused onto the light-sensitive surface and converted into an electrical signal by the camera electronics system.

Selection of a camera and lens for a video assessment system should start with the determination of the degree of resolution (the ability to see fine details of an image) to be required. Three levels of increasing resolution may be considered:

- detection: resolution that provides the ability to detect the presence of an object in the area of interest

- classification: resolution that provides sufficient information to determine object class (animal, blowing debris, man)

- identification: improved resolution sufficient to uniquely identify an object on the basis of details of appearance

These three levels of resolution depend on size and proximity of the object in question. For example, it might be possible to identify a person at close range, classify an object as a large dog or a man at an intermediate distance, and detect an object's presence at a far distance. In addition the size of an object affects the resolution because at a given distance large objects can be identified, smaller objects may be classified and still smaller objects may just be detected.

The camera selection should also take into consideration the following desirable characteristics:

- high sensitivity to best utilize available light

- ability to retain picture clarity at all points in the scene when motion is present

- ability to maintain an adequate picture in the presence of bright sources

- long life

\subsection{Types of Cameras}

\subsubsection{Image Tube Cameras}

In the image tube camera, the light-sensitive surface is scanned by an electron beam from the lower right side of the imaging surface horizontally across the surface to the left side. The beam is then relocated just above the first, line and at the right side. The second line is also scanned from right to left. This scanning process is repeated until the scanning beam has reached the top of the light-sensitive surface. (See Figure 2-1.) The beam then returns to the bottom of the image tube, and the same scanning pattern is repeated, except that the second scan traces a path between the lines of the first scanning pattern. This method of electron-beam scanning is termed interlaced scanning because the second field scanning lines are inter laced between the scanning lines of the first field. The scanning pattern on the video monitor is the cpposite of the camera scanning pattern. The electron beam begins at the upper left of the monitor and scans from left to right and top to bottom. (See Figure 2-2.) Interlaced scanning reduces flicker in the displayed image. The scanning pattern itself is called a raster.

As the electron beam scans the light-sensitive surface, the beam is modulated by the surface in proportion to the amount of light at each point on the light-sensitive surface. The modulated beam then becomes the video signal whose amplitude is proportional to the amount of light at a given point. Each scan from bot tom to top is called a field, and two fields combine to form a video frame. In U.S. standards (RS-170), a field is produced 60 times per second, and a complete frame of video is produced 30 times per second. F.ach field enntains 262.5 horizontal scan lines, and each frame contains 525 horizontal scan lines. 


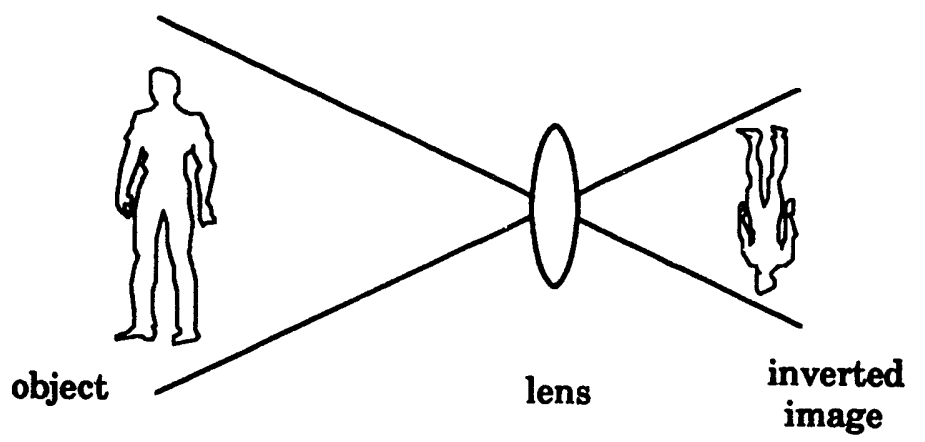

Camera scanning pattern: right to left and bottom to top

Monitor scanning pattern: left to right and top to bottom
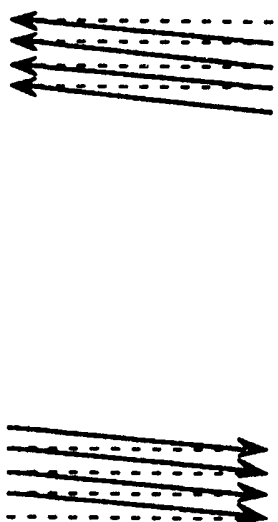

Figure 2-1. Scanning

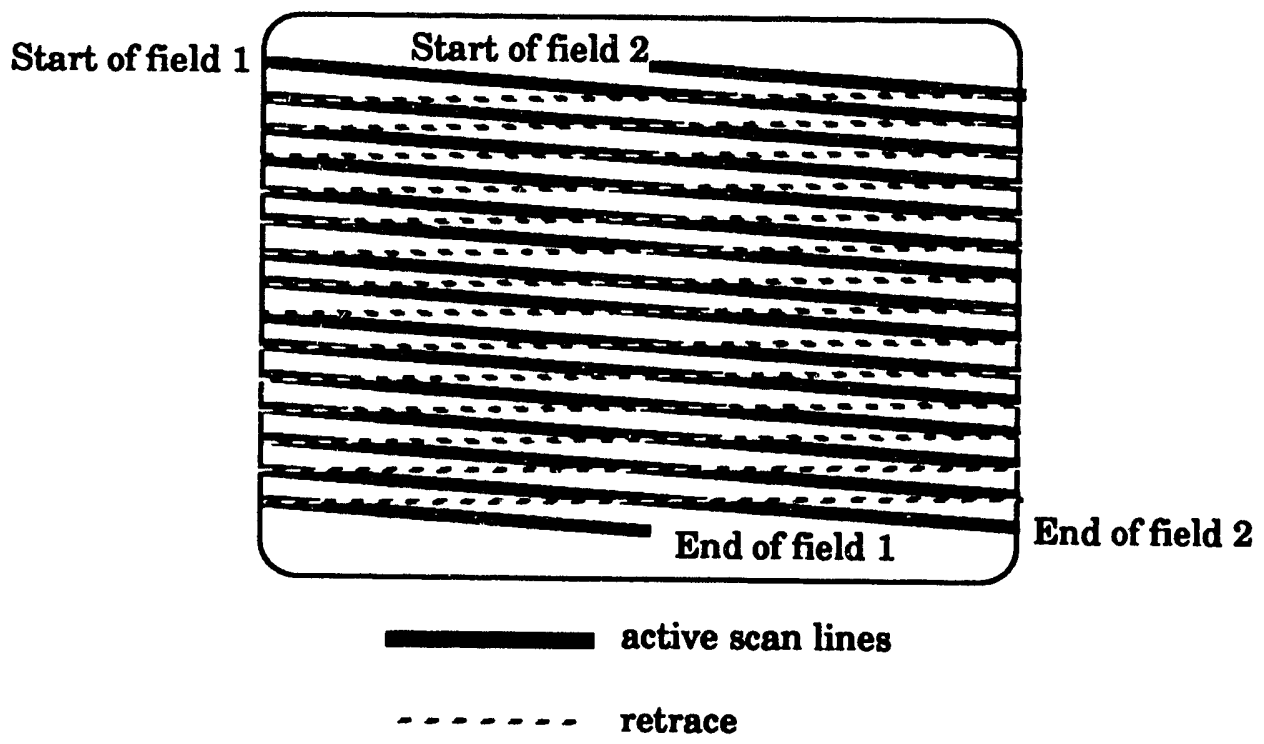

Figure 2-2. Interlaced scanning

Each horizontal line is scanned in approximately 63.5 microseconds. Since the beam returns to the right side of the imaging surface before each line, the beam is cut off to allow the deflection system to reposition the beam. Figure 2-3 illustrates a voltage waveform created by this scan proportional to light on one horizontal line. This beam cut-off period is called horizontal blanking, or retrace time, and lasts approximately 11 microseconds. Similarly, the beam is cut off as it returns from the top to the bottom of each field. This vertical blanking interval lasts approximately 1.27 milliseconds or the equivalent duration of 20 horizontal scan lines. Only 485 scanning lines of the raster contain video information, and they are called active scan lines. 


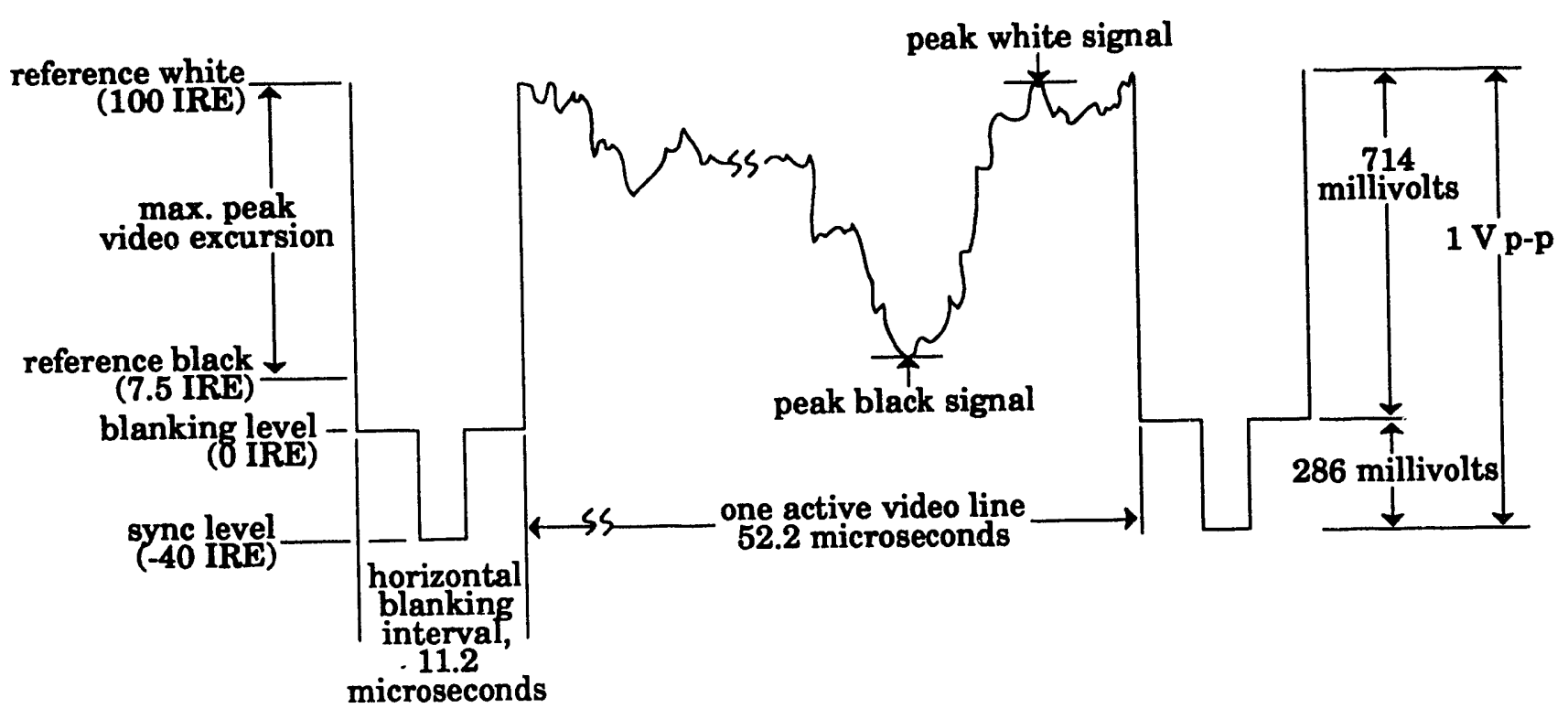

Figure 2-3. EIA Standard RS-170 video waveform

There are three classes of image tubes that provide usable video at different illumination levels:

- Standard image tubes are used in daylight and well-lighted interior scenes.

- Low light level image tubes are used in daylight down to well-lighted exterior scenes (1.0 footcandle [fc]).

- Intensified image tubes are used for dimly lighted scenes.

Standard image tubes, or vidicons, having an antimony trisulfide photosensitive surface, are used primarily in daylight and well-illuminated interiors. They have a spectral sensitivity similar to that of the human eye. Their sensitivity can be controlled by varying the potential on an internal element, the target, which allows the vidicon to handle several orders of magnitude in illumination. The standard vidicon exhibits a degree of image retention, resulting in image smear if either the camera or an objact in the field of view is in motion. This image smear, called comet-tail or lag, is expressed in the percentage of signal existing three fields ( 50 milliseconds) after illumination is removed. The figure of third-field lag for the standard vidicon is about 20 percent, meaning that at the end of the third field after the illumination is removed, 20 percent of the original signal amplitude remains at that point on the imaging surface. One of the primary limitations of standard vidicons in security applications is their susceptibility to image burn-in when operating in fixed positions for extended periods of time. Burn-in degrades resolution and sensitivity and eventually requires replacement. The primary advantage of standard vidicons is their lower cost.

Low light level cameras typically use image tubes having greater sensitivity, wider spectral response, and greater resistance to image burn-in than standard vidicons. One such image tube is the silicon diode array vidicon whose photosensitive imaging surface is composed of discrete photodiodes. This image tube has a greater sensitivity than the standard vidicon. The silicon array has a somewhat reduced resolution from the standard vidicon, but significantly reduced third-field lag. It has considerable sensitivity to the near infrared spectrum and is extremely resistant to image burn-in. The silicon array tube has no means of controlling its sensitivity to large variations in light intensity. The light reaching the light-sensitive surface should be controlled externally either by neutral density filters or, more commonly, by automatically controlled iris mechanisms in the lens. The most popular current method of light control lenses uses a combination of auto-iris electromechanical control with an internal neutral gradient density spot in the center of the lens. This combination provides the dynamic range of light levels required 
for full daylight to low light level operation. Silicon diode array tubes are available under the following commercial trade names: Epicon, Sidicon, Sivicon, S-T Vidicon, Tivicon, and Ultricon.

A second type of low light level image tube is the hetero-junction type, which has a light-sensitive surface of cadmium and zinc tellurides. It has a resolution slightly less than the standard vidicon and is somewhat more sensitive to visible light than the silicon array tube. It is considerably more resistant to image burn-in than the standard vidicon, but will retain an image under very high light levels in a fixed position for a long time. Like the silicon array tube, the hetero-junction type of image tube requires external control of the illumination reaching the light-sensitive surface. The hetero-junction image tubes are more commonly called by their trade name Newvicon.

Intensified versions of these three types of image tubes improve the light sensitivity considerably. Because of their image-intensifier sections, their resolution and signal-to-noise ratio are somewhat reduced. The inherent sensitivity to near infrared energy of the silicon array tube, and to a lesser extent of the Newvicon, is significantly reduced by the limitations of the image-intensifier sections of these image tubes. Intensified image tubes are also susceptible to image burn-in at the intensifier section.

The option of using low light level cameras assisted by artificial lighting for nighttime assessment has frequently been chosen over the use of very low light level intensified image tube cameras for the following reasons:

- significantly reduced camera procurement and maintenance costs

- reduced probability of image tube damage in high brightness conditions

- increased resolution and signal-to-noise ratio

- additional psychological deterrent effect of nighttime illumination

- improved visibility for a security response force

\subsubsection{Solid-State Imager Cameras}

Over the past several years, solid-state light-sensitive imaging devices have been developed. Only recently have they been developed with resolution and sensitivity that make them competitive with the image tube. While still not possessing the same specifications as the better image tubes, their arrival in the commercial market has created a new era in video imaging. Even the least expensive of the new family of solid-state image devices has been used in interior assessment, with the expectation of increased reliability and reduced maintenance requirements. Presently, only a few solid-state imagers have the sensitivity to be used with moderate illumination from exterior artificial lighting.

A solid-state imaging device is made up of a rectangular array of discrete silicon cells that are inherently immune to image burn-in. Within the imaging device, the conversion from light to an electrical signal can be done by any of the following types of solid-state devices:

- charge coupled device (CCD)

- charge injection device (CID)

- charge primed device (CPD)

- metal oxide silicon (MOS) imager

These devices vary from each other in the manner in which the electrical charge is read from the lightsensitive surface and in the read-out method. The CCD is the device most commonly found in commercially produced video cameras at this time. As the device name implies, the electrical charge freed by the optical absorption is coupled out of the array's photosensitive layer by a set of controlled transfer (shift) registers into the output stage. Frame transfer and interline transfer are the two basic CCD image architectures.

The solid-state imager does not require an electron beam to create a video signal. The charge from the light-sensitive cells is read out serially in the same sequence as the raster is scanned in the image tube, producing the same two fields of video interlaced to form a single video frame. 
The image chip is only a few centimeters in size, as compared to the typical 15-cm (6-in.) length of the standard image tube. Power consumption is significantly reduced by the absence of the heated cathode found in the image tube. The solid-state imaging camera also contains a sync generator to control the timing of the readout of the image cells to create a composite video signal output.

There are also intensified versions of solid-state cameras for use in very low light level applications (less than $1.0 \mathrm{fc}$ ). These cameras use light amplification techniques to enhance the image sensed by the video imager.

\subsection{Sync Generation}

The sync (synchronizing) generator for the camera controls the time and duration of the horizontal and vertical sync pulses in the deflection system and the blanking pulges in the video section of the camera. The sync pulses determine the start of the beam retrace time, and the blanking pulses determine the timing of the vertical and horizontal blanking intervals.

There are several nodes of camera sync generator operation. Genlock operation produces the utmost in sync stability and is standard where monitor vertical roll cannot be tolerated when switching between video sources. Genlock operation requires a master sync and distribution system to each video device in the system. The major disadvantage is cost. Some cameras are designed to default to either line-lock or crystal operation in the event of the loss of the genlock (sync) signal.

Line-lock operation may be considered as an inexpensive substitute for a master sync system, i.e., synchronous operation without a master sync generator or sync distribution system. The sync generator in each camera operated from alternating current (AC) power will adjust its frequency and phase to the zero-crossing of the AC line voltage. All cameras should be on the same phase as the AC line or should contain phase adjustment circuitry to compensate for the different possibilities in the connection to three-phase power systems. As long as the zero-crossingjitter is reasonably small, no monitor roll should result when switching between cameras.

In crystal operation, each camera operates from its own internal, free-running sync generator. Direct current (DC)-powered cameras without external sync should be operated in the crystal mode. This mode provides the greatest opportunity for monitor roll when switching between video sources. Some of the effect of switching between non-synchronous sources can be reduced by careful monitor seiection. Camera selection should be based on the sync generator configuration required by all of the individual components in the complete video assessment system. Many modern components recognize the framing pulse and automatically lock on sync at the beginning of the next cycle.

\subsection{Sensitivity}

The sensitivity of a television camera can be defined as the minimum amount of illumination required to produce a specified output signal. The following factors are involved in producing a TV signal:

- illuminance level of the scene

- spectral distribution of the illumination source

- object reflectance

- total scene reflectance

- camera lens aperture

- camera lens transmittance

- spectral response of the camera imager

- video amplifier gain, bandwidth, and signal-to-noise ratio

- electronic processing circuitry

Camera sensitivity is usually specified as the minimum illuminance level that will produce a full 1 volt peak-to-peak video signal. (See Figure 2-3.) The specification should state whether the indicated illuminance level is the scene illuminance or the faceplate illuminance. The illumination source is usually an incandescent lamp operating at a color temperature of $2,854 \mathrm{~K}$. In some cases, parameters 
are inappropriately used by manufacturers to claim better performance. Two of the favored parameters are higher scene reflectances than are normally encountered and greater transmittance than is commonly available in standard auto-iris lenses with neutral density spot filters.

The amcunt of illumination incident upon the imager faceplate determines the performance for a given camera imager and is defined as:

$$
I_{F}=\frac{I_{S R T}}{4 f^{2}(1+m)}
$$

where:

$I_{\mathrm{F}}=$ faceplate illuminance in footcandles

$I_{8}=$ scene illuminance in footcandles

$\mathbf{R}=$ scene reflectance in percent

$T_{L}=$ lens transmittance in percent

f $\quad$ f-number of lens

$\mathrm{m}$ = magnification: image size/object size (The value for $\mathrm{m}$ is insignificant in assessment applications.)

\subsection{Scene Ilumination}

An understanding of the relative levels of scene illumination produced by natural sources, the amount of light reflected from typical scenes, and the resultant faceplate illumination levels required by the variety of image tube and solid-state imagers is important to the successful deployment of even the simplest closed-circuit television (CCTV) system. Table 2-1 provides the approximate illumination levels that exist under natural lighting.

Table 2-1. Scene illumination levels

\begin{tabular}{|lr|}
\hline Full Sun & 1,000 to $10,000 \mathrm{fc}$ \\
\hline Overcast & 10 to $1,000 \mathrm{fc}$ \\
\hline Twilight & 0.1 to $10 \mathrm{fc}$ \\
\hline Full moon (clear) & 0.01 to $0.1 \mathrm{fc}$ \\
\hline Full moon (overcast)* & 0.001 to $0.01 \mathrm{fc}$ \\
\hline Starlight (clear) & 0.0001 to $0.001 \mathrm{fc}$ \\
\hline Siarlight (overcast)* & 0.00001 to $0.0001 \mathrm{fc}$ \\
\hline
\end{tabular}

*Man-made illumination in inhabited and/or industrial areas will produce higher illumination levels because of reflection from the clouds 
The percentage of light reflected from a scene (reflectance) depends on the incident light angle and on the texture and composition of the reflecting surface. For natural illumination, the reflectance of various scenes is relatively independent of the angles of incidence and reflection. Table 2-2 lists some common surfaces and their approximate reflectances.

îable 2-2. Scene reflectance

\begin{tabular}{|c|c|}
\hline Surface & Reflectance (\%) \\
\hline Empty asphalt surface & $7-10$ \\
\hline Sandy soil, wet & $15-20$ \\
\hline Grass-covered area with trees & $20-25$ \\
\hline Red brick building & $30-35$ \\
\hline Sandy soil, dry & $30-35$ \\
\hline Unpainted concrete & $35-40$ \\
\hline Smooth surface aluminum & $60-65$ \\
\hline Snow-covered field & 70-75 \\
\hline
\end{tabular}

\subsection{Resolution}

Resolution is the ability to see fine details in an image. It is a measure of spatial frequency or the number of pairs of alternate black and white evenly sized lines that can be seen in a given linear distance, typically expressed in line pairs per millimeter. The line pairs designation is used primarily in the field of optics, but the term appears occasionally in television literature.

The resolution of a TV camera is commonly measured on an Electronic Industries Association (EIA) resoiution chart where groups of equally spaced black and white lines arranged in a wedge-shaped pattern form the basis for resolution measurement. (See Figure 2-4.) A camera is positioned so that it views the full chart only with no background visible. At some point along the horizontal converging black and white lines, the lines merge into a solid gray area. The point where the lines barely appear separate is the limiting vertical resolution. It is specified in TV lines of resolution and represents the number of independently resolved black and white lines (or elements) in the full vertical field of view. The resolution chart is marked at various intervals along the wedge patterns with the resolution values in TV lines, typically between 200 and 1,600 lines.

Since the TV image is produced by scanned raster lines, the resolution in TV lines is often confused with the number of scanned raster lines that produce the image. Although the vertical resolution in TV lines is dependent upon the number of scanning lines in the raster, they have different meanirgs.

The 525 horizontal scan line system used in the United States has 485 active scan lines containing video information. Only about 340 TV lines can be individually resolved because of the deflection system nonlinearities in the camera and the monitor. The $340 \mathrm{TV}$ lines can also be thought of as 340 independent picture elements of equal height, stacked one above the other in a vertical column. Each of these picture elements, or pixels, is of a specific, measurable vertical dimension representing one-three hundred fortieth of the resolution chart height. The pixel is defined as the smallest independently resolvable element in an image. 


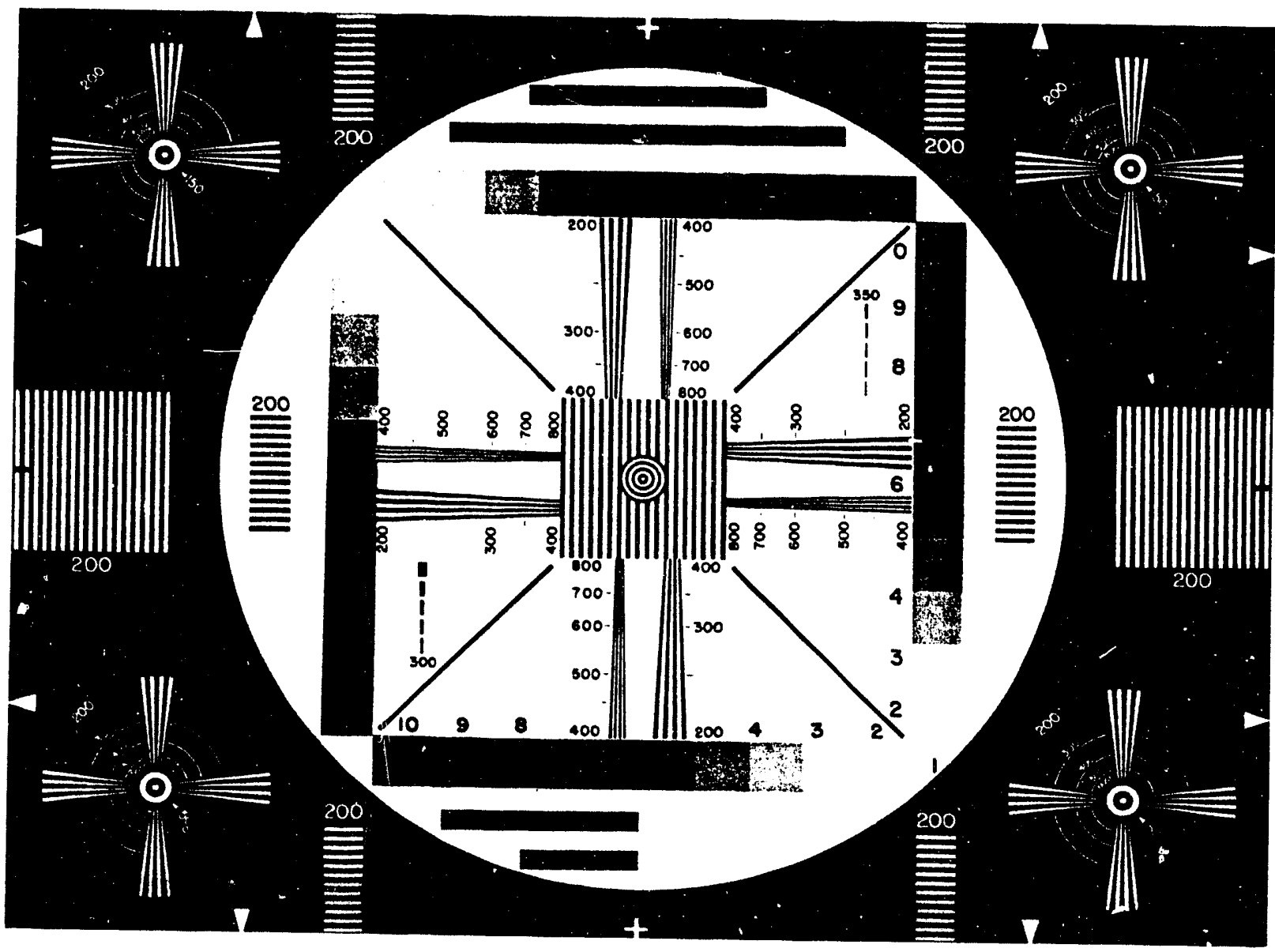

Figure 2-4. EIA resolution chart

There are several ways of specifying the height of objects relative to the vertical field of view. For example, an object in the camera's field of view having a height that subtends 10 horizontal scanning lines on a video monitor can also be said to have a height of seven $(=10 \times 340 / 485)$ TV lines of resolution or seven pixels. There is some appeal to using scanning lines because they can actually be counted on the monitor; however, TV lines of resolution and pixel count have more meaning when horizontal resolution is considered.

Horizontal resolution can be measured using the EIA resolution chart with the vertical black and white wedge pattern in the same way. The point where the converging vertical lines are just barely visible before fusing into a gray blur is defined as the limiting horizontal resolution in TV lines. The width of the lines on the chart are the same dimension at any given resolution point both horizontally and vertically. Therefore, the horizontal resolution read from the chart is not defined by the width of the picture but by a distance equal to the picture height or three-fourths of the picture width. If the horizontal and vertical resolution are the same, then the pixel dimensions will be equal in height and width. If the horizontal resolution is twice the vertical resolution, then the pixel will be twice as high as it is wide.

The horizontal field of view is greater than the vertical field of view by 33 percent. If the vertical and horizontal resolution were 300 TV lines each, there would be 400 pixels in the horizontal direction, but the horizontal resolution would still be 300 TV lines by definition.

As previously stated, the vertical resolution is primarily dependent upon the number of horizontal scanning lines. In monolithic, photosensitive-surface image tube cameras, the horizontal resolution is primarily dependent upon the bandwidth of the camera. On cameras with solid-state imagers, the horizontal resolution is determined by the number and spacing of the discrete elements in the horizontal dimension. In most cases, the horizontal resolution in TV lines or pixels equals the number of discrete elements in three-fourths of the scene width. The practical method for determining resolution is to read 
the resolution from the EIA resolution chart as in previous examples. This will provide a common basis for the performance comparison among various types of cameras.

The currently available production solid-state cameras have horizontal limiting resolutions in the 250 to $575 \mathrm{TV}$ line range, as compared to the 600 to $800 \mathrm{TV}$ line range in the 1 -in. thermionic image tube cameras. In the solid-state imager, there is no deflection system required to scan the photosensitive surface. Therefore, there are no deflection errors to contribute to a loss in vertical or horizontal resolution from this source. The vertical resolution typically increases from about 340 TV lines in image tube cameras to about $400 \mathrm{TV}$ lines of vertical resolution in solid-state imager cameras.

Frequently only one resolution specification is listed in manufacturers' literature for image tube cameras. The listed specification should be assumed to be the horizortal resolution because the vertical resolution is fixed by the number of horizontal scanning lines that is established for each national system standard.

\subsection{Camera Formats}

The size of the imaging area is determined by the amplitude of the beam deflection drive signals in the image tube and by the size of the photosensitive surface. The area of the image on the photosensitive surface defines the image tube format size. A 1-in. (nominal) diameter image tube has an image size of $0.500 \mathrm{in}$. wide by $0.375 \mathrm{in}$. high $(12.7 \mathrm{~mm}$ by $9.525 \mathrm{~mm})$. The diagonal (corner-to-corner) measurement is $0.625 \mathrm{in}$. (15.88 $\mathrm{mm}$ ). The ratio of width to height is called the aspect ratio, expressed as a four to three aspect ratio. All common video imaging equipment, cameras and monitors, are standardized on this four to three aspect ratio. This particular image tube size has a format that is variously and interchangeably called a 1-in. format (from the tube diameter) or the $16 \mathrm{~mm}$ format, referring to the diagonal measurement of the image area. This image tube size is the most commonly used in high-quality assessment applications because of its high resolution and sensitivity.

The next most popularly used image tube format is the $2 / 3$-in. format. This image tube has a nominal diameter of $0.690 \mathrm{in}$. $(17.6 \mathrm{~mm})$. The actual image size on the photosensitive surface is $0.346 \mathrm{in}$. wide by $0.259 \mathrm{in}$. high ( $8.8 \mathrm{~mm}$ by $6.6 \mathrm{~mm}$ ). The diagonal measurement is $0.432 \mathrm{in}$. (11.0 $\mathrm{mm}$ ). This image tube format is identified as the $2 / 3-i n$. or the $11 \mathrm{~mm}$ format. The smaller image size results in a reduced sensitivity and resolution image. This image tube is used in less expensive cameras for interior surveillance and assessment and in entry-control booths for personnel identification.

A third image tube format, rarely used in assessment applications, is the $1 / 2-\mathrm{in}$. ( $8 \mathrm{~mm}$ ) format. The image size is $0.244 \mathrm{in}$. wide by $0.183 \mathrm{in}$. high $(6.20 \mathrm{~mm}$ by $4.65 \mathrm{~mm})$. The diagonal measurement is $0.305 \mathrm{in}$. (7.75 $\mathrm{mm}$ ). The lower resolution and sensitivity parameters of this format size render it unacceptable for highquality perimeter assessment applications.

While some solid-state imaging arrays in large sizes have been produced for special applications, the more common format size for solid-state imaging cameras is the $2 / 3$-in. format. A few manufacturers have produced $1 / 2$-in. format chips, and these are found to a lesser extent in the commercially produced cameras for security applications. The image sizes are $8.8 \mathrm{~mm}$ by $6.6 \mathrm{~mm}$ for the $2 / 3$-in. format solid-state cameras and $6.20 \mathrm{~mm}$ by $4.65 \mathrm{~mm}$ for the $1 / 2$-in. format cameras. There is some variation in the standard format size for $1 / 2$-in. imagers.

Table 2-3 summarizes camera image format sizes.

\subsection{Camera Location}

An exterior assessment camera should be located so as to give a full view of the entire sensor detection volume. If sensor elements are above ground, the camera field of view should include the sensor hardware in order to assess a sensor tampering event and an intrusion alarm. When sensors are located below ground, the camera should be located to include the entire detection volume and any sensor hardware, such as tamper-protected sensor junction boxes, that may be mounted above ground. 
Table 2-3. Camera image format sizes

\begin{tabular}{|llll|} 
Format & $\begin{array}{l}\text { Horizontal } \\
\mathrm{mm} \\
\text { (in) }\end{array}$ & $\begin{array}{l}\text { Vertical } \\
\mathrm{mm} \\
\text { (in) }\end{array}$ & $\begin{array}{l}\text { Diagonal } \\
\mathrm{mm} \\
\text { (in) }\end{array}$ \\
\hline \begin{tabular}{llll|}
$16 \mathrm{~mm}$ \\
$(1 \mathrm{in})$.
\end{tabular} & $\begin{array}{l}12.7 \\
(0.500)\end{array}$ & $\begin{array}{l}9.525 \\
(0.375)\end{array}$ & $\begin{array}{l}15.88 \\
(0.625)\end{array}$ \\
\hline $\begin{array}{l}11 \mathrm{~mm} \\
(2 / 3 \text { in.) }\end{array}$ & $\begin{array}{l}8.8 \\
(0.346)\end{array}$ & $\begin{array}{l}6.6 \\
(0.259)\end{array}$ & $\begin{array}{l}11.0 \\
(0.432)\end{array}$ \\
\hline $8 \mathrm{~mm}$ & 6.20 & 4.65 & 7.75 \\
$(1 / 2$ in.) & $(0.244)$ & $(0.183)$ & $(0.305)$ \\
\hline
\end{tabular}

In many installations, a protected facility uses two chain-link funces around the perimeter with a clear area between the two fences. This provides a well-defined sensor and assessment area. The primary requirement of the assessment camera system is to display as much of the detection area as possible, specifically the clear zone between the fences and the outside of the inner fence perimeter barrier. Camera and lens selection and positioning should ensure detection and classification of any visible cause of sensor alarms regardless of the time of day. For these reasons, it is important that the following criteria are observed:

- The spacing between the inner and outer fences (under optimal conditions) should be uniform.

- Minimum and maximum clear zone width restrictions should be considered.

- Type of surface of clear zone between fences should be carefully selected.

- Grading and sterilization of the clear zone should be performed.

- Adequate area illumination level and uniformity should be provided.

Deviations from these criteria will generally reduce system efficiency and increase overall system and maintenance cost by increasing equipment requirements to achieve acceptable system effectiveness.

Cameras should point roughly parallel to the fence lines to provide good views of the clear zone. They can be positioned at any point between the inner and outer fences, but there are some practical restrictions in that they should not interfere with the sensor and should not be able to be used as sensor bridging aides. If the camera is mounted along or above the inner fence, the camera would perceive the inner fence as a solid wall. If barbed wire outriggers are used on top of the fence, as is common practice, this wall effect is extended and provides a place for an intruder to hide after crossing the sensored area. A slightly delayed assessment would not indicate that an intrusion had taken place. For this reason, the mounting of cameras above the inner fence should be minimized.

Cameras located just inside the outer fence slightly angled into the facility can see through much of the inner fence, thus eliminating hiding places along the inner fence. A slightly delayed assessment should reveal an intruder at, or attempting to cross, the inner fence.

Camera mounting heights of 4.5 to $9 \mathrm{~m}$ (15 to $30 \mathrm{ft}$ ) are typical. Low mounting heights are convenient for camera installation and maintenance, but the distance perspective is poor. Higher mounting levels provide a better distance perspective along the clear zone and avoid the problem of viewing bright sky above the horizon, but accessibility is more difficult. Higher mounting is preferred, but care should be taken during system design, particularly when using lenses of $75 \mathrm{~mm}$ or longer focal lengths. The vertical field of view is so small that the maximum effective sector length may be reduced, particularly if the ground slopes upward between the camera and the far end of the assessment sector. Calculation of the fields of view is discussed in the section on lenses. 


\subsection{Camera Mounting}

Assessment cameras are usually mounted on steel towers. Freestanding triangular steel towers are preferred both for their stability and for their siting possibilities. Because they are nearly transparent due to their lattice-like construction, they can be located within the assessment zone without providing a possible hiding place. Positioned near the outer fence, cameras can be angled slightly in ward to provide a good view of the inner fence. In this location, the tower may provide an aid for bridging the outer fence but should not provide an aid for bridging the intrusion sensors.

The freestanding tower, as its name implies, has no guy wires for support. It is attached to a base buried in concrete several feet below ground level. The location of the tower is important in minimizing the windinduced tower vibration being coupled into the ground, possibly causing nuisance alarms in some types of sensors.

Attachment of the assessment camera to the tower or pole can be accomplished in many ways. The primary consideration should be the mounting rigidity. The length of the attachment arm should be as short as possible to reduce wind-induced motion. The mount should allow adjustment for optimum positioning of the camera. The adjustment mechanism should be as simple and as rugged as possible for maximum rigidity.

Cameras should be installed so that no light sources are directly in the field of view. Direct light can cause blooming of the image or allow the auto-iris lens circuitry to reduce the lens aperture. Possible sources include perimeter lighting, building security lighting, objects capable of reflecting light, and the sky. Viewing portions of the sky should be avoided to eliminate camera blinding at dusk and dawn when the sky is considerably brighter than the ground, regardless of which direction the camera is facing. Cameras should never be angled to look above the horizon. Considerable care should be taken because camera blinding from unexpected sources is difficult to predict prior to installation and is one of the most frequent problems later encountered. When there is a choice, north/south perimeter sections should have the cameras facing north to minimize sun reflections at low sun angles. Illumination sources in the field of view may have to be reoriented and/or shielded to prevent camera blinding. A perimeter section adjacent to a roadway presents problems from vehicle headlights and taillights, even when the roadway is a considerable distance from the assessment camera.

Mounting the camera in an unprotected area could lead to undetected camera tampering. To detect camera tampering, the video line can be electronically supervised. Video presence detectors, which can monitor the video signal and produce an alarm if the video level increases or decreases by a preset amount, are commercially available. The video presence detector can also detect when the sync signal amplitude has been reduced.

\subsection{Environmental Housings}

Video cameras in an exterior environment require protection from temperature extremes and from precipitation. There are two types of enclosures to protect the cameras from the elements. The first type is the integral environmental housing that forms the outer shell of the camera. It is quite rigid and sturdy and can be pressurized with dry nitrogen and equipped with thermostatically controlled integral heaters. A sun shade can be attached to overhang the lens and deflect light or precipitation from the glass faceplate. In high winds, the faceplate may not remain entirely free of precipitation, and some compromise in visual assessment can be expected.

The second option is to mount a camera inside a separate sheet-metal or fiberglass housing, which permits access to the camera by a hinged or removable lid. These housings can be equipped with heaters, insulation, fans, defrosters, windshield washers, and windshield wipers. All of these functions can be automatically controlled at the housing except the windshield washers and wipers. The washers and wipers should be remotely controlled from the central assessment station.

These separate housings should be large enough to contain the camera, lens, and cable connectors. Their chief advantage is the accessibility to the camera for adjustments, such as lens focusing. They cannot be 
pressurized, so some dirt and dust accumulation can be expected inside these housings. The windshield washers and wipers have proved to be a considerable maintenance problem, and the washers are not recommended unless very unusual environmental conditions exist. Washer reservoirs, which should be located at the camera, compel more frequent access to the camera location. The windshield wipers, if kept properly aujusted and free of ice, can maintain adequate assessment in high precipitation situations where the nonwipered camera faceplate may have problems with assessment. The conditions at each site would determine the need for these devices in light of their aaded maintenance requirements.

The separate environmental housing will require additional wiring to provide the power necessary to operate the electrical equipment. A remote control system will be required for the operation of windshield wipers and washers, if v sed. These remote control systems can be furnished by the housing manufacturer or are available from other manufacturers, frequently also with remote control of other camera-related functions such as pan/tilt mounts and zoom lenses.

\subsection{Pan/Tilt Mounts}

Pan/tilt camera mounts may be effectively used in surveillance applications where remote control of the camera's position and pointing angles is desirable. In limited cases, the unit can provide a useful backup to assessment situations if properly located. These mounts are not normally used in place of fixed focal length assessment cameras that have been carefully placed to provide the maximum assessment value. Use of pan/tilt systems compromises effective, timely assessment.

Pan/tilt systems typically provide horizontal (pan) positioning of $350^{\circ}$ and vertical positioning (tilt) of plus or minus $90^{\circ}$ from the horizontal. Limit switches protect the cables by restricting the allowable motion. Some more expensive pan/tilt mounts can provide unlimited travel in the pan mode by the addition of electrical slip rings. Their higher cost is usually not acceptable.

Pan/tilt mounts are rated by the maximum allowable load they can position and by the speed of rotation. Three load ranges from under $4.5 \mathrm{~kg}(10 \mathrm{lb})$ up to $36 \mathrm{~kg}(80 \mathrm{lb})$ can be accommodated in popular pan/tilt mounts. Their rotational speed is limited to about 4 to 6 degrees per second for the less expensive units. Faster units cost more, but their controllability at high speeds is limited by the operator's orientational perceptions from a remote location. One major disadvantage of the pan/tilt system is the requirement for continuous operator attention while operating such a system. No other activities can be monitored by an operator while the pan/tilt unit is in motion. Some newer models of pan/tilt mounts allow programming preset aim points that provide a quicker and more accurate assessment of points of interest than manual searching.

\subsection{Interior Applications}

Camera layouts for interior assessment follow the same principles and guidelines as for exterior cameras. Lighting levels suitable for human comfort and safety ( 30 to $100 \mathrm{fc}$ ) will be adequate for most cameras. The inexpensive $11 \mathrm{~mm}(2 / 3-i n$.) format cameras, either image tube or solid-state imager cameras, will be acceptable from a performance stardpoint. Resolution is normally not a significant factor because of the limited range of operation in most interior applications. A solid-state imager camera should provide better reliability.

If image tube cameras are selected, the silicon array or Newvicon types have advantages over standard vidicons to avoid the image burn-in problems over extended periods of time. In reasonably constant illumination levels, a lens with a manually controlled iris is less expensive than an auto-iris lens. If assessment is to be carried out in reduced light levels, such as in off-shift operations, then the auto-iris lens should be used. If a master sync system is planned for the entire assessment system, the camera selected should be capable of accepting external sync signals.

Cameras mounted at the corners of a room just below the ceiling usually provide the best assessment. Corners away from entry points are preferred in order to eliminate camera tampering from someone below the camera and out of the camera field of view. Wide angle lenses can be used to provide full wallto-wall $\left(90^{\circ}\right)$ camera coverage. 
Tilted downward, the camera avoids the ceiling lights that would adversely affect the camera signal. This still allows viewing over the lower office and/or laboratory equipment. When tall equipment is located in the room, a second camera may be required to observe the blind side. If a second camera is required, a good location may be the corner diagonally across from the first camera.

\subsection{Camera Selection}

Camera selection should be based primarily on the sensitivity required for a full video output signal in the lighting environment in the area to be assessed. Within any given classification of camera imager, there may be only subtle differences in performance between manufacturers. A Newvicon from any source should perform within a limited range of sensitivity. Solid-state imagers seem to have a broader range of sensitivities within a given class, such as the CCD imager. This is primarily the result of cell size, readout method, and manufacturing techniques. The sensitivity should match the lighting design goals, regardless of the imager.

The resolution of the imager is next in importance because it will determine the number of cameras required for a given straight line perimeter section. The greater the resolution, the greater the spacing between the cameras can be. The object resolution required should be determined before the camera selection, but in practice, the desired object resolution may be slightly modified when the possible camera choices are limited.

Camera format is an important consideration in the camera selection process. The format size determines the sensitivity of the image tube, with smaller formats having reduced sensitivity as well as lower resolution. The trade-off in this situation is price, but the cost of the camera is only part of total system cost. If $11 \mathrm{~mm}(2 / 3-i n$.) tube cameras are selected instead of $16 \mathrm{~mm}$ (1-in.) format tubes, the illumination level may have to be increased, driving up the cost of installing more luminaires with continually higher electrical usage for the life of the system.

Format size also affects the field of view, which dictates the numbe: of lenses available in a variety of focal lengths. The requirements of special design lenses for nonstandard focal lengths should be considered and evaluated at considerable length before committing to such action.

The most common tube cameras are the $16 \mathrm{~mm}$ (1-in.) format silicon array or Newvicon image tube types. They possess the greatest range of sensitivity and resolution of the image tube class. Their long-term use in many applications has established a good data base for their use.

In the newer solid-state imagers, it is evident that the $11 \mathrm{~mm}(2 / 3-i n$.$) format will be the most popular and$ will provide the greatest variety of choices. Only a very few of the currently ai:rilable solid-state imagers approach the sensitivity of the $16 \mathrm{~mm}$ (1-in.) image tubes, but they do not yet have quite the resolution of the image tubes. The selection of a good CCD camera requires considerable evaluation because they have only been in use a short time.

During the selection process, evaluation of production cameras should be undertaken in the real lighting environment expected at the site. In many cases, the experience of other facilities can help to reduce the number of options considered. Manufacturers' literature should not be the sole criteria in camera selection. The specifications, or the conditions under which specifications are developed, may be totally unrealistic in relation to the design problem at hand.

Other considerations in the selection process should include the difficulty in maintenance, the packaging of the camera regarding the environment in which it will be used, the maintenance support from the manufacturer, and the documentation supporting the equipment. Documentation should include operating, adjustment, and maintenance procedures; theory of operation; block diagrams; schematics; and manufacturer and commercial replacement parts lists. Serious consideration should be given to eliminating any manufacturers' product that does not include this documentation.

When specifying a camera for purchase, the EIA standards are helpful in determining the critical parameters. EIA Standard RS-170 is frequently quoted by manufacturers even though this standard 
applies to television studio facilities. The significant part of this specification is the requirement for equalizing pulses in the vertical blanking interval. Outwardly, EIA Standard RS-330 would appear to be the specification of choice since it lists the performance standards for CCTV cameras. This specification leaves, as optional, the use of equalizing pulses, which could be cause for misinterpretation.

A good reference for aid in specifying cameras is EIA Standard RS-312, which is not a camera specification itself, but an outline for specifying camera performance.

\subsection{Acceptance Testing}

An incoming inspection should be made of any cameras purchased for evaluation or for the final system installation. Obviously. different parameters will be evaluated for the two situations. Evaluation cameras will be compared to other cameras purchased for the same purpose. Upon receiving cameras for the final installation, camera performance should be evaluated to determine conformity with the manufacturers' specifications, compatibility with the design criteria, and consistent performance from camera to camera. Experience has shown that final inspection at the manufacturers' plant is not consistent, and performance may deviate considerably from the specifications. Frequently some equipment has been damaged or has had parts shaken loose in transit. Operating the equipment for a few hundred hours continuously before final installation usually decreases the maintenance problems during the installation phase of perimeter construction. Any problems discovered at this point, should be referred to the manufacturer for resolution while still under warranty.

\subsection{Maintenance}

With incoming inspe:tion and an equipment test phase prior to installation, maintenance problems should be minimized for the short term. Camera adjustment will probably consume most of the maintenance time.

Optical focus of the camera lens has consistently been a major time-consuming factor in the original installation. The day-to-night illumination levels and energy spectrum changes are responsible for most of these problems. Optical focus is more reliable if accomplished at night under the appropriate scene lighting from the final camera location. Cameras in sealed environmental housings typically pose a serious restriction to this procedure, and many attempts have been made to circumvent or substitute this procedure with others. Cameras in sealed or pressurized housings should be night focused by the manufacturer or user. Then a defective unit in the field is replaced by a good camera/housing assembly, and troubleshooting of this bad unit is conducted in an electronics shop.

Maintenance problems are best resolved by a competent on-site staff capable of understanding the complexities and interrelationships of all of the concepts used in the original system design as well as having a background in electronic systems troubleshooting. Specific, periodic maintenance requirements should come from the equipment manufacturer in the form of printed documentation.

A supply of spare parts should be stocked according to the manufacturers' recommendations and eventually supplemented by on-site experience. A lab for maintenance should be provided with test equipment suitable for camera adjustment and repair.

\subsection{Documentation}

Manufacturers' equipment documentation shculd be preserved at the using site as well as at a central document storage location. Any equipment modifications made on-site should also be documented and stored at these two locations.

A maintenance log of all camera repairs and adjustments should be kept to provide a historical record of each piece of equipment. Maintenance trends can be established to identify recurring problems and equipment failures. This practice will substantially reduce repair time and identify any equipment performing in a substandard manner. 


\section{LENSES}

The lens of a video camera focuses the light reflected from an object onto the imaging surface of the camera where it is converted to an electrical video signal. In general, the main purpose of lens selection will be to cover as much of the desired area as possible with a minimal number of cameras while retaining an acceptable degree of overall resolution.

\subsection{Focal Length and Field of View}

Focal length is the single most important factor in proper lens selection. The focal length of a lens describes its relative magnification: the ratio of the image size to the object size. The image size is directly proportional to the focal length so that the image produced by a $100 \mathrm{~mm}$ lens will be twice the size of the image produced by a $50 \mathrm{~mm}$ lens. This also means that the linear field of view (width and height) is proportional to the focal length. The focal length will determine the horizontal and vertical angular fields of view for a given lens format. It should be noted that the angular field of view is not directly proportional to focal length.

The field of view is dependent upon the lens focal length and the camera imager format. It should be noted that not all lens manufacturers and suppliers agree on the format size of the $1 / 2$-in. format imagers, so some variations to these figures may appear in literature from different sources. Lenses of the same focal length from different sources may also claim different angular fields of view. As these variations are usually small, this should not create a significant problem for the video system designer. It should be understood that there is a manufacturing tolerance of as much as 4 percent in the actual focal length of the lenses produced from the same design. This tolerance should be considered by the video system designer when planning site layouts.

For a desired minimum resolution, the range (length of the coverage) of the short focal-length lens will be less than that of the long one, as Figure 3-1 illustrates. The sectors indicate the usable camera coverage.

Table 3-1 lists the vertical and horizontal angular fields of view for some of the more common focal length lenses used on cameras having the indicated formats. Tables 3-2, 3-3, and 3-4, found on the following pages, give the horizontal linear fields of view of some common lenses at various distances from the camera.

\subsection{Aperture}

The relative aperture, or speed, of a lens is a measure of its ability to gather light and is expressed as the $f$-number. The $f$-number is the ratio of the lens focal length to its clear aperture. For example, an $f / 2$ lens has a clear aperture diameter of one-half of its focal length, so that an $f / 2$ lens with a focal length of $50 \mathrm{~mm}$ has a clear aperture of $25 \mathrm{~mm}$. It should be noted that the clear aperture is not always the diameter of the outer (objective) lens, but a measure of the image diameter.

The actual light-gathering capability is not always apparent by knowing the f-number alone. Lenses are constructed with various numbers of imaging elements, usually with -arious types of glass. Each glassto-air interface causes some entering light to be scattered raticer 'inan passing through the element. Typically, 3 percent to 4 percent of the light is scattered rather than transmitted at each interface. Antireflective coatings can be applied to the lens surfaces to reduce the amount of scattering. While two differently designed lenses may have the same focal length and f-number, the same amount of light may not be transmitted through both lenses. A typical example may be found in variable focal length lenses, or zoom lenses, which have a large number of glass elements, some of which are moveable. In this case, an $\mathrm{f} / 2$ zoom lens will not transmit as much light as an $\mathrm{f} / 2$ fixed focal length lens. This fact should be taken into account when selecting cameras and lenses and when designing lighting systems. 
Limits Imposed by Resolution Requirements

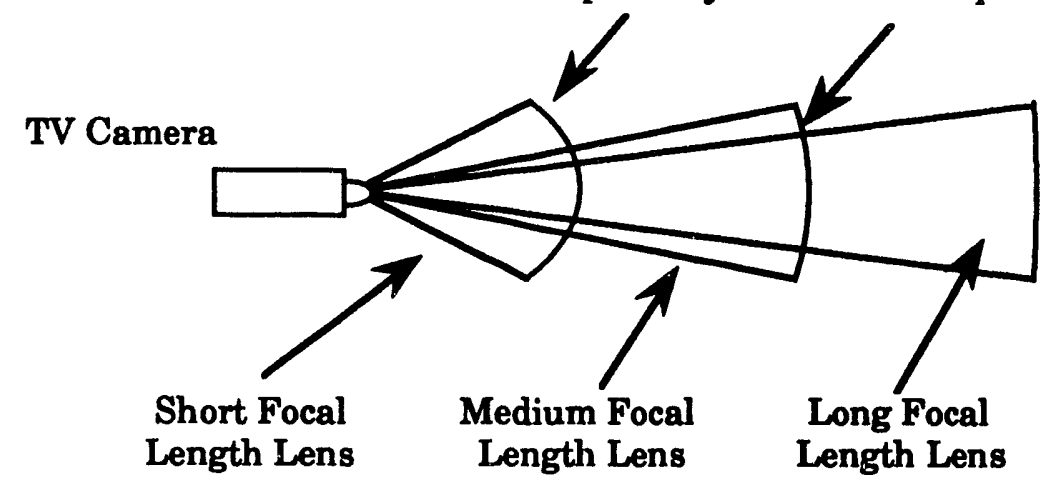

Figure 3-1. Practical field of view vs. focal length of lens

Table 3-1. Angular fields of view (degrees)

\begin{tabular}{c|c|c|c|c|c|c|}
\multirow{4}{*}{$\begin{array}{c}\text { Focal } \\
\text { Length } \\
(\mathrm{mm})\end{array}$} & \multicolumn{2}{c|}{$\begin{array}{c}16 \mathrm{~mm}(1 \mathrm{in}) \\
\text { horiz }\end{array}$} & \multicolumn{2}{c|}{$\begin{array}{c}11 \mathrm{~mm}(2 / 3 \text { in. }) \\
\text { vert }\end{array}$} & \multicolumn{2}{c|}{$\begin{array}{c}8 \mathrm{~mm}(1 / 2 \text { in. }) \\
\text { horiz }\end{array}$} \\
\hline 4.8 & 105.8 & 79.4 & 85.0 & 63.8 & 65.7 & 49.3 \\
\hline 5.7 & 96.2 & 72.1 & 75.3 & 56.5 & 57.1 & 42.8 \\
\hline 6.5 & 88.7 & 66.5 & 68.2 & 51.1 & 51.0 & 38.2 \\
\hline 8.0 & 76.9 & 57.7 & 57.6 & 43.2 & 42.4 & 31.8 \\
\hline 8.5 & 73.5 & 55.1 & 54.7 & 41.1 & 40.1 & 30.1 \\
\hline 9.0 & 70.4 & 52.8 & 52.1 & 39.1 & 38.0 & 28.5 \\
\hline 12.5 & 53.9 & 40.4 & 38.8 & 29.1 & 27.9 & 20.9 \\
\hline 16.0 & 43.3 & 32.5 & 30.8 & 23.1 & 21.9 & 16.4 \\
\hline 25.0 & 28.5 & 21.4 & 20.0 & 15.0 & 14.1 & 10.6 \\
\hline 35.0 & 20.6 & 15.4 & 14.3 & 10.7 & 10.1 & 7.59 \\
\hline 50.0 & 14.5 & 10.9 & 10.1 & 7.54 & 7.10 & 5.32 \\
\hline 75.0 & 9.68 & 7.26 & 6.72 & 5.04 & 4.73 & 3.55 \\
\hline
\end{tabular}

$$
\stackrel{\circ}{h_{\text {fov }}}=2 \operatorname{TAN}^{-1} \frac{W_{I}}{2 \mathrm{FL}}
$$

Where: $\quad h_{\text {for }}^{\circ}=$ horizontal angular field of view (degrees)

$\mathrm{W}_{\mathrm{I}}=$ width of active scan area (mm) of imager (Table 2-3)

FL = lens focal length (mm)

(Note: Vertical angular field of view $=0.75 \mathrm{~h}^{\circ}{ }_{\text {sor }}$ ) 
Table 3-2. Horivontal linear fields of view for focal length from $16 \mathrm{~mm}$ (1-in.) camera format

\begin{tabular}{|c|c|c|c|c|c|c|c|c|c|c|c|c|}
\hline \multicolumn{13}{|c|}{$16 \mathrm{~mm}$ (1-in.) Format } \\
\hline D FL & 8.8 .8 & 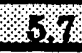 & 6.6. & 8.0. & $8,5$. & $9 \times 0$ & 10,5 & $16 \%$ & 2.3. & $35:$ & 50 & $75 \%$ \\
\hline 10. & 26 & 22 & & & & & & & & & & \\
\hline 20 & 53 & 45 & 39 & 32 & 30 & 28 & & & & & & \\
\hline 30 & 79 & 67 & 59 & 48 & 45 & 42 & 30 & & & & & \\
\hline 10. & 106 & 89 & 78 & 64 & 60 & 56 & 41 & 32 & & & & \\
\hline 00 & & 111 & 98 & 79 & 75 & 71 & 51 & 40 & & & & \\
\hline 60 & & & 117 & 95 & 90 & 85 & 61 & 48 & 30 & & & \\
\hline 70. & & & & 111 & 105 & 99 & 71 & 56 & 36 & & & \\
\hline 80 & & & & & 120 & 113 & 81 & 64 & 41 & 29 & & \\
\hline 90 & & & & & & & 91 & 71 & 46 & 33 & & \\
\hline 100 & & & & & & & 102 & 79 & 51 & 36 & & \\
\hline 110 & & & & & & & 112 & 87 & 56 & 40 & & \\
\hline 120. & & & & & & & 122 & 95 & 61 & 44 & 30 & \\
\hline 140 & & & & & & & & 111 & 71 & 51 & 36 & \\
\hline 160 & & & & & & & & & 81 & 58 & 41 & \\
\hline 180 & & & & & & & & & 91 & 65 & 46 & 30 \\
\hline 200 & & & & & & & & & 102 & 73 & 51 & 34 \\
\hline 2220 & & & & & & & & & 112 & 80 & 56 & 37 \\
\hline 240 & & & & & & & & & & 87 & 61 & 41 \\
\hline 260 & & & & & & & & & & 94 & 66 & 44 \\
\hline 280 & & & & & & & & & & 102 & 71 & 47 \\
\hline 300. & & & & & & & & & & 109 & 76 & 51 \\
\hline 350 & & & & & & & & & & & 89 & 59 \\
\hline 400 & & & & & & & & & & & 102 & 68 \\
\hline 450 & & & & & & & & & & & 114 & 76 \\
\hline 500 & & & & & & & & & & & & 85 \\
\hline 550 & & & & & & & & & & & & 93 \\
\hline 600 & & & & & & & & & & & & 102 \\
\hline 6.50 & & & & & & & & & & & & 110 \\
\hline
\end{tabular}

$$
h_{\text {fov }}=\frac{12.7 D}{F L}
$$

where:

$$
\begin{aligned}
& \mathrm{h}_{\text {fov }}=\text { horizontal field of view, in feet (or meters) } \\
& \mathrm{D}=\text { distance from camera to object, in feet (or meters) } \\
& \mathrm{FL} \quad=\text { lens focal length, in millimeters }
\end{aligned}
$$


Table 3-3. Horizontal linear fields of view for focal length from $11 \mathrm{~mm}(2 / 3-i n$.$) camera format$

\begin{tabular}{|c|c|c|c|c|c|c|c|c|c|c|c|c|}
\hline \multicolumn{13}{|c|}{$11 \mathrm{~mm}(2 / 3$-in.) Format } \\
\hline D FL & 18 & 5.7 & 6.5 & 8.0 & 8.5 & 9.0 & 12.5 & 16 . & 25 & 35 & 50 & 76 \\
\hline 10. & 18 & 15 & & & & & & & & & & \\
\hline 20 & 37 & 31 & 27 & 22 & & & & & & & & \\
\hline 30 & 55 & 46 & 41 & 33 & 31 & 29 & & & & & & \\
\hline 80 & 73 & 62 & 54 & 44 & 41 & 39 & 28 & 22 & & & & \\
\hline 50 & 92 & 77 & 68 & 55 & 52 & 49 & 35 & 28 & & & & \\
\hline 60 & 110 & 93 & 81 & 66 & 62 & 59 & 42 & 33 & & & & \\
\hline $\mathbf{7 0}$ & & 108 & 95 & 77 & 72 & 68 & 49 & 39 & & & & \\
\hline 80. & & & 108 & 88 & 83 & 78 & 56 & 44 & 28 & & & \\
\hline 90 & & & & 99 & 93 & 88 & 63 & 50 & 32 & & & \\
\hline 100 & & & & 110 & 104 & 98 & 70 & 55 & 35 & & & \\
\hline 110 & & & & & & 108 & 77 & 61 & 39 & & & \\
\hline 120 & & & & & & & 84 & 66 & 42 & 30 & & \\
\hline 140 & & & & & & & 99 & 77 & 49 & 35 & & \\
\hline 160 & & & & & & & 113 & 88 & 56 & 40 & 28 & \\
\hline 180 & & & & & & & & 99 & 63 & 45 & 32 & \\
\hline 200 & & & & & & & & 110 & 70 & 50 & 35 & \\
\hline 220 & & & & & & & & & 77 & 55 & 39 & \\
\hline 240 & & & & & & & & & 84 & 60 & 42 & 28 \\
\hline 260 & & & & & & & & & 92 & 65 & 46 & 31 \\
\hline 280 & & & & & & & & & 99 & 70 & 49 & 33 \\
\hline 300 & & & & & & & & & 106 & 75 & 53 & 35 \\
\hline 850 & & & & & & & & & & 88 & 62 & 41 \\
\hline 400 & & & & & & & & & & 101 & 70 & 47 \\
\hline 450 & & & & & & & & & & 113 & 79 & 53 \\
\hline 500 & & & & & & & & & & & 88 & 59 \\
\hline 600 & & & & & & & & & & & 106 & 70 \\
\hline 700 & & & & & & & & & & & & 82 \\
\hline 800 & & & & & & & & & & & & 94 \\
\hline 900 & & & & & & & & & & & & 106 \\
\hline
\end{tabular}

$$
h_{\text {fov }}=\frac{8.8 D}{F L}
$$

where:

$$
\begin{aligned}
& \mathrm{h}_{\text {fov }}=\text { horizontal field of view, in feet (or meters) } \\
& \mathrm{D} \quad=\text { distance from camera to object, in feet (or meters) } \\
& \mathrm{FL} \quad=\text { lens focal length, in millimeters }
\end{aligned}
$$


Table 3-4. Horizontal linear fields of view for focal length from $8 \mathrm{~mm}$ (1/2-in.) camera format

\begin{tabular}{|c|c|c|c|c|c|c|c|c|c|c|c|c|}
\hline \multicolumn{13}{|c|}{ 8mm (1/2-in.) Format } \\
\hline D FL & $8 \% 8.8$ & $8.1 \%$ & $6.6 \%$ & $8.0 \%$ & 8.6. & 9.0 .0 & 10,5 & 16 & 25 & 35 & 60 & 76 \\
\hline 20 & 26 & 22 & & & & & & & & & & \\
\hline 30 & 39 & 33 & 29 & 23 & & & & & & & & \\
\hline 80 & 52 & 44 & 38 & 31 & 29 & 28 & & & & & & \\
\hline 80 & 65 & 54 & 48 & 39 & 36 & 34 & & & & & & \\
\hline 60 & 78 & 65 & 57 & 46 & 44 & 41 & 30 & 23 & & & & \\
\hline 20. & 90 & 76 & 67 & 54 & 51 & 48 & 35 & 27 & & & & \\
\hline 80 & 103 & 87 & 76 & 62 & 58 & 55 & 40 & 31 & & & & \\
\hline 90. & & 98 & 86 & 70 & 66 & 62 & 45 & 35 & & & & \\
\hline 100 & & 109 & 95 & 78 & 73 & 69 & 50 & 39 & & & & \\
\hline 120 & & & 114 & 93 & 88 & 83 & 60 & 47 & 30 & & & \\
\hline 140 & & & & 109 & 102 & 96 & 69 & 54 & 35 & & & \\
\hline 160 & & & & & 117 & 110 & 79 & 62 & 40 & 28 & & \\
\hline 180 & & & & & & & 89 & 70 & 45 & 32 & & \\
\hline 200 & & & & & & & 99 & 78 & 50 & 35 & & \\
\hline 2220 & & & & & & & 109 & 85 & 55 & 39 & & \\
\hline 240. & & & & & & & & 93 & 60 & 43 & 30 & \\
\hline 250 & & & & & & & & 97 & 62 & 44 & 31 & \\
\hline 260 & & & & & & & & 101 & 64 & 46 & 32 & \\
\hline 280 & & & & & & & & 109 & 69 & 50 & 35 & \\
\hline 300 & & & & & & & & & 74 & 53 & 37 & \\
\hline 350 & & & & & & & & & 87 & 62 & 43 & 29 \\
\hline 80.0 & & & & & & & & & 99 & 71 & 50 & 33 \\
\hline 500 & & & & & & & & & 124 & 89 & 62 & 41 \\
\hline 600 & & & & & & & & & & 106 & 74 & 50 \\
\hline 700 & & & & & & & & & & & 87 & 58 \\
\hline 800 & & & & & & & & & & & 99 & 66 \\
\hline 900 & & & & & & & & & & & 112 & 74 \\
\hline 1000 & & & & & & & & & & & & 83 \\
\hline 1100 & & & & & & & & & & & & 91 \\
\hline 1200 & & & & & & & & & & & & 99 \\
\hline 1300 & & & & & & & & & & & & 107 \\
\hline
\end{tabular}

where:

$$
\begin{aligned}
& \mathbf{h}_{\text {fov }}=\text { horizontal field of view, in feet (or meters) } \\
& D \quad=\text { distance from camera to object, in feet (or meters) } \\
& \text { FL = lens focal length, in millimeters }
\end{aligned}
$$




\subsection{Depth of Field}

The lens depth of field is defined as the distance between the nearest and farthest object whose images are in proper focus. Depth of field will increase by increasing the $f$-number (decreasing aperture) and is dependent on the quality of the lens as defined by its resolution. The hyperfocal distance is defined as the distance at which a lens is focused to produce acceptable focus from half that distance to infinity. For example, a sample lens of a given aperture that produces sharp images from $50 \mathrm{~m}(164 \mathrm{ft})$ to infinity would have a hyperfocal distance of $100 \mathrm{~m}(328 \mathrm{ft})$. This is a unique number for each lens and is also a function of the resolution of the lens.

\subsection{Format}

The lens format, like the camera imager format, is the maximum usable image created by the lens. Standard lens formats are the $16 \mathrm{~mm}$, or 1-in., format (with an imaging diameter of about $16 \mathrm{~mm}$ ) used on the 1-in. format camera tubes and the $11 \mathrm{~mm}$, or $2 / 3$-in., format (with an imaging diameter of $11 \mathrm{~mm}$ ). The 2/3-in. format lenses cannot be used on 1-in. format imaging devices without losing a considerable portion of the image. However, the 1-in. format lenses can be used on $3 / 3$-in. format imagers with an imaged scene overflowing the $3 / 3$-in. imaging format.

\subsection{Lens Resolution}

The resolution of the lens does not ordinarily limit the overall assessment system response. Some manufacturers may quote resolution in TV lines of resolution, typically $\mathbf{8 0 0}$ TV lines or more. It is generally understood that lenses are designed to optical line pairs per millimeter standards which have significantly greater resolution than any other component in the video system.

\subsection{Light Control}

As previously described in the camera section, only the standard vidicon can internally adapt to wide variations in light levels. All of the other commonly used cameras contain imagers that require external control of the incoming illumination. By far the most widely used method of light control is found in the magnetically operated automatic iris lens system. Usually the video signal amplitude is monitored, and a drive signal is created to maintain the amplitude at some predetermined, adjustable level. As the video level decreases, the auto-iris lens is driven to increase the aperture. At some point, however, the iris is driven fully open, and a decreasing light level will result in a reduced amplitude video signal.

Some cameras are designed with an automatic gain control (AGC) circuit to maintain the video signal level as the light is reduced. However, the AGC circuit adds additional noise to the video signal. In tube imagers, the low light levels and AGC result in a reduced resolution and signal-to-noise ratio. In solidstate imagers, the signal-to-noise ratio decreases, but the resolution remains fairly constant. The resulting image at the lower light levels is compromised by the narrow dynamic range between black and white levels (reduced contrast) until the image becomes unusable.

During the installation of cameras, the cameras should be focused to produce the sharpest image under the lowest light conditions expected. This will produce the best overall focus setting because under a fully open iris condition depth of field is minimum.

\subsection{Variable Focal Length Lenses}

Variable focal length, or zoom, lenses are quite popular with facility security designers. Zoom lenses seem to possess a unique opportunity to provide considerable flexibility in solving assessment problems; however, they usually fail in this regard. Zoom lenses have a limited role in assessment applications because of the variable focal length. A properly designed assessment area does not have an infinite number of possible focal lengths that can effectively be used. It would be unusual if two different standard focal length, auto-iris lenses could be used in the effective assessment of any given perimeter sector. 
Because there is a limited selection of lenses with focal length more than $25 \mathrm{~mm}$, manual zoom lenses with auto-iris lenses may cost three to five times more than a fixed focal length lens. They could be used in an unusual geometry sector where there are severe limitations on the possible camera location, and the optimum focal length is greater than $25 \mathrm{~mm}$ but is not a standard focal length. Zoom lenses have more lens elements, which results in reduced light transmissivity, requiring additional artificial lighting for night assessment. Remote control of the focal length in a zoom lens adds to the cost over a manual zoom lens. This cost is in the range of five to eight times that of a fixed focal length lens and may exceed the cost of the camera.

The most common and preferred use of the zoom lens is in surveillance applications where a pan/tilt camera mount is also used. In this configuration, a pan-tilt-zoom (PTZ) equipped camera could be used for assessment backup in the event of camera failure. This would permit the orderly replacement of the failed camera without leaving the assessment area unprotected. It should be noted that this camera would not be accessible for other (surveillance) activities while it is being used for assessment backup. The location of the PTZ camera should be such that assessment is feasible from that location, and the zoom range is set to achieve video coverage equivalent to the failed camera.

\subsection{Object Resolution}

In viewing a complex scene, objects of various sizes should be detectable. Small objects may or may not be seen, depending on their positions vertically at the image plane of the camera. Studies have shown that reliable object detection occurs when the object subtends 1.5 to 2.5 pixels; object orientation, 2.1 to 3.5 pixels; object classification, 6.4 to 9.5 pixels; and object identification, 10 to 16 pixels.

Defining the minimum resolution for a specific application depends on the confidence level of the specifier. Persons less familiar with their own or others' abilities to detect a given object may specify higher resolution requirements. Poor lighting, complex scenes, low scene reflectance, expected maintenance problems, and other factors may also tend to encourage the use of higher resolution requirements. For these reasons many different minimum resolution standards have been developed for different applications over several years.

None of these standards are wrong, but higher resolution requirements mean greater numbers of cameras should be used where extended straight-line perimeter sections should have continuous assessment coverage. The resolution in pixels (or scan lines) per foot is inversely proportional to the camera-to-object maximum distance; thus, greater resolution requirements mean a greater number of cameras.

If the assessment scene is viewed live from the monitor, then all of the resolution capability of the video system is available to the viewer. If the scene is recorded on magnetic tape or in solid-state memory, then the resolution is restricted by the tape recorder to about $300 \mathrm{TV}$ lines of horizontal resolution or by the solid-state memory to about 360 TV lines for a 512 by 512 memory array.

Allowing for all of the system restrictions, image recording, and environmental factors, a resolution of 300 TV lines of resolution horizontally and vertically can be used as a conservative guide for system design. For a 1-in. format image tube, $300 \mathrm{TV}$ line horizontal and vertical resolution and four pixels per foot object resolution, a maximum horizontal field of view of $30.48 \mathrm{~m}(100 \mathrm{ft})$ is acceptable. The 30.48 $\mathrm{m}(100-\mathrm{ft})$ width restriction is applicable regardless of the lens focal length selected. The minimum-sized object will then subtend 1 percent of the horizontal field of view.

For real-time assessment, the horizontal resolution of the camera, usually about 600 TV lines, can be used as a reference. A common standard of eight pixels per foot of object resolution would indicate that the maximum horizontal field of view should be limited to $30.48 \mathrm{~m}$ (100 ft), or 1 percent of the horizontal field of view. While this standard ignores the fact that the vertical resolution is limited to only 340 to 400 TV lines, the result is the same as the four pixels per foot object resolution based on a 300 TV line maximum horizontal and vertical system resolution stated above. From this, it can be seen that the object resolution specification in pixels per foot should include the system resolution used as a basis for this specification. 
Object resolution can be measured in the field using a variety of one-foot shapes, such as a circle, square, and triangle. In this test the video monitor observer should be able to classify which shape(s) has been placed at the far end of the assessment zone. Another test target for measuring horizontal resolution is a one-foot square with a number (corresponding to resolution) of alternating white and black bands. In this test the observer should note whether or not the alternate bands are discernible.

\subsection{Site Layout}

Site layout can begin as soon as a preliminary sensor layout has been defined. If a standard, double-fence perimeter is required, the assessment widths are usually determined by the fence boundaries. Templates can be fabricated for the fields of view for common standard focal length lenses. The templates can be fabricated from flexible clear plastic cut to the desired angle with the aid of a protractor. Overlaying a site plan drawing of any scale factor, the templates will indicate the camera fields of view for the selected focal lengths. The templates can be marked with distance references relating to the scale of the site drawing, if desired.

A table of maximum distances for each of the lenses to be used can be generated for reference as the design process continues. The maximum distances depend on the object resolution determined to be adequate for the specific site. Calculations on the minimum distance to the point where the lens can see the full spacing between fences should be made for each lens to be considered. Thus, the minimum and maximum distances that a specific lens can cover can be established for that particular site.

For the special case where an assessment system is being designed for perimeter use, the distance and width approximation may be used to determine the maximum zone length that may be assessed with a particular camera and lens combination. Figure 3-2 shows a typical perimeter configuration. Note that the lower field of view (bottom of scene on TV monitor) is not always the minimum zone width. Likewise, the upper field of view is not normally the limiting field of view width. Also note that between the camera location and lower field of view, there is a blind area which cannot be seen by the camera.

If standard lenses $(25 \mathrm{~mm}, 50 \mathrm{~mm}, 75 \mathrm{~mm}$, etc.) are to be used, a fast and easy calculation can be made to determine either the width of the field of view at a given distance from the camera or the distance from the camera given a specific width. Either of these, distance or width, can be approximated as follows:

$D \cong 2\left(\frac{F L}{25}\right) h_{\text {fov }}$ for 1 -inch camera format

$D \cong 3\left(\frac{F L}{25}\right) h_{f o v}$ for $2 / 3$-inch camera format

$D \cong 4\left(\frac{F L}{25}\right) h_{\text {fov }}$ for $1 / 2$-inch camera format

$D=$ distance from camera (m)

$\mathbf{F L}=$ focal length $(\mathrm{mm})$

$h_{\text {fov }}=$ width of field of view (m)

As an example of distance and width approximation, a $20 \mathrm{~m}$ wide area, assessed with a $16 \mathrm{~mm}$ format camera using a $50 \mathrm{~mm}$ focal length lens, would have its camera placed $80 \mathrm{~m}$ away in order to see the entire $20 \mathrm{~m}$ width. 

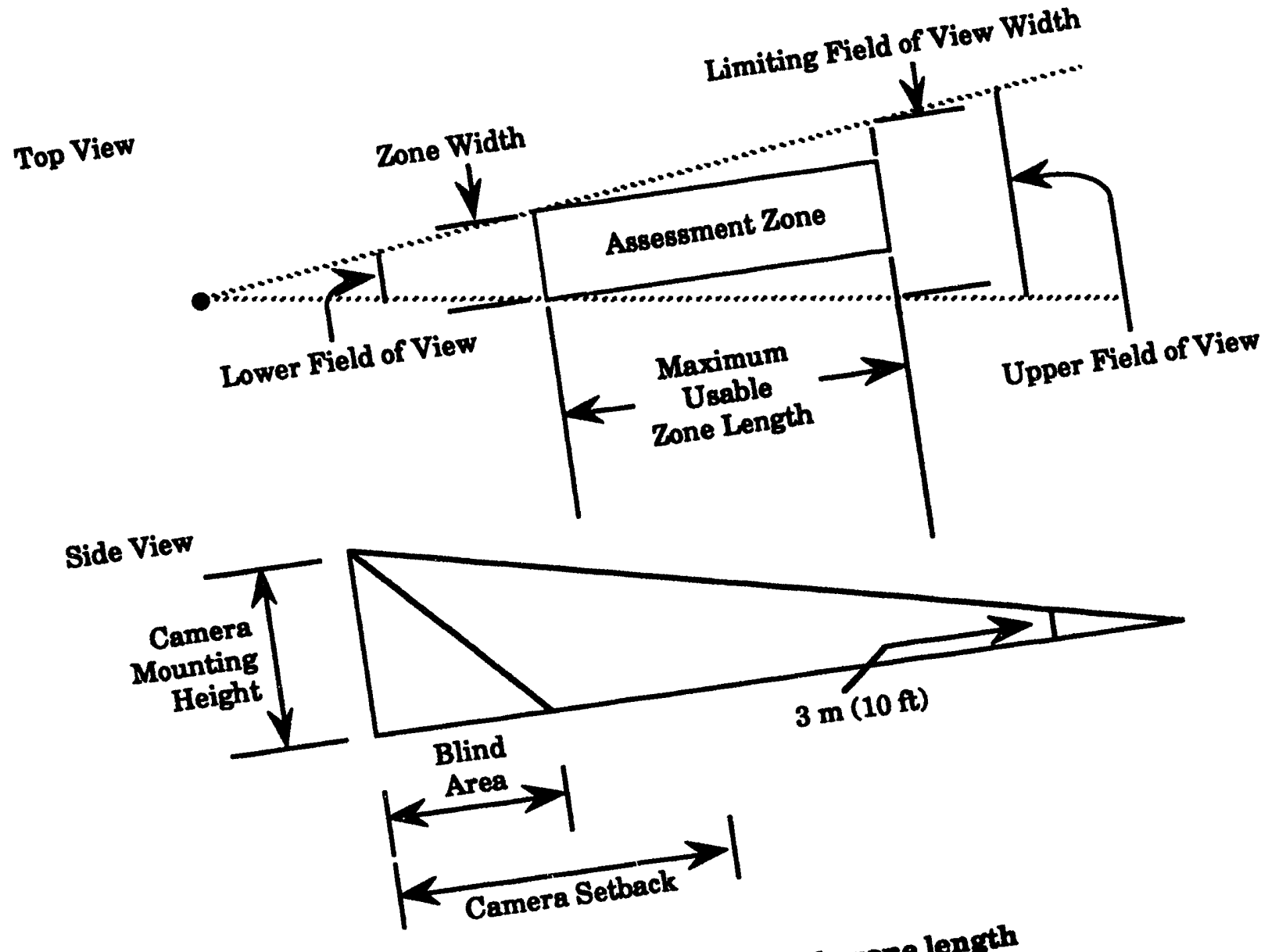
the distance to the limiting field of view width can also be It is located where Using the equation above, the dised on experimental data to recomple, the distance frem The resolution requiremen distance. the horizontal camera would be $120 \mathrm{~m}$.

calculated based on zone width and resolution require width distance The maximum usable zone length is calculate limiting field of view lens, and $16 \mathrm{~mm}$ format, the math lens is end of the zone. It is the difference bety $20 \mathrm{~m}$ wide zone width, 50 is easy to determine whiches as a $20 \mathrm{~m}$ wide, $50 \mathrm{~m}$ For the example used premeters. Using this tecample, if the requirement wo would be required. to the zone. usable zone length is socific zone size. For work. A longer focal and the closest camera location to the a spect of view the best suited for a $50 \mathrm{~mm}$ lens would adequate zone coverage and then a wise to select a lens that provides ad The camera tilt should satisfy two condidth. At the far end of the assessent). should cover its full assess over an 8-foot fence (10 feet). cover a person climbing The usual procedure is to start in any ce direction should to keep all the came in fenciner fence (the protected The usual procedure is to stange in fence direction is desirable to keep all inside
break. Any significant chany installations, it is a have to be mounted
only a few degrees. For mance lengths, a camera may side of the facility). 
The templates and the minimum and maximum usable distances for each lens are then used to continue the trial and error layout process around the facility perimeter. Although the discussion assumes a double-fence perimeter, any sensored open area is handled in the same way. The boundaries are the limits set by agreement, usually slightly larger than the sensored area, rather than the obvious fence lines.

\subsection{Procurement}

Lenses are procured only after all the site assessment design layouts have been made and confirmed. Camera manufacturers typically offer a fairly complete line of manual and auto-iris lenses for their cameras. Most cameras and lenses use a standard $C$ mount attachment method. The $C$ mount has a 25.4 $\mathrm{mm}$ (1-in.) thread diameter with 32 threads per in. The standard $\mathrm{C}$ mount lens has its image plane 17.5 $\mathrm{mm}(0.69$ in.) behind the lens mounting flange surface. While some manufacturers may have $a$ lf.ns and camera mount different from the standard $\mathrm{C}$ mount with very limited selection of lens focal lengths, they may offer a $\mathbf{C}$ mount adapter. All lenses purchased should have the standard $\mathrm{C}$ mounting configuration for standardization, interchangeability, and the widest range of focal lengths.

The auto-iris lens should be selected for all cameras except standard antimony trisulfide vidicons. For quicker response, lack of motor noise, and extended iris life, the preferred auto-iris lens is magnetically driven rather than motor driven. To accommodate the wide range of light levels expected in all exterior mounted cameras, the auto-iris lenses should be equipped with a neutral density spot filter. Only interior mounted cameras operating with constant scene illumination can be expected to operate without autoiris lenses.

There are only about six to eight focal lengths from which to choose among all manufacturers of autoiris, neutral-density, spot-filter lenses. This narrows the options in system design. For this reason, it is very important to establish a working relationship with sensor and site construction designers to provide a complete security system that considers all of the factors and limitations.

\subsection{Maintenance}

No maintenance is required with the auto-iris lens. All repair work should be referred to the manufacturer. The outer (objective) lens can be cleaned with an alcohol/water mix or lens cleaner and a lint-free optical cloth or lens tissue. Abrasive liquids, pastes, or cloths should be avoided. Care should be exercised in cleaning to prevent scratching the lens surface and/or coatings. Except under unusual circumstances, the lens should not be removed to clean the rear lens element or the imager surface. 


\section{LIGHTING SYSTEMS}

Correctly designed lighting systems are of paramount importance in the performance of any video assessment system. Even low light level cameras will not provide adequate assessment capabilities unless careful attention is given to all aspects of lighting needs, including a basic understanding of lamp types and characteristics, as detailed below.

\subsection{Lamp Types}

Primary lamp types include

- Incandescent: Light is emitted from a heated filament inside an evacuated globe.

- Quartz Iodine: Light is generated as in an incandescent lamp, but the globe is filled with a halogen gas that regenerates the filament and allows higher intensity.

- Fluorescent Lamp: Light is generated by electric current conducted by ionized mercury vapor inside a glass tube. The low pressure vapor emits ultraviolet radiation that is converted to visible light by fluorescent powders on the inner surface of the tube.

- High Intensity Discharge Lamp: The light energy is generated by direct interaction of an arc with the gas to produce visible light. High intensity discharge lamps include mercury vapor lamps, metal halide lamps, and high and low pressure sodium lamps. Argon is normally added to aid starting, and various powders or vapors may be added to improve color rendition.

Table 4-1 summarizes various lamp characteristics.

\subsection{Lighting Requirements}

The four major requirements in lighting specification ar?

(1) Horizontal illumination-Light striking a horizontal plane is termed horizontal illumination. The intensity of this light should be sufficient to provide adequate faceplate illumination to the camera. This is a function of the camera imaging device, the lens speed (f-number), lens quality (transmissivity of the lens itself), and the ground surface. The equation for faceplate illumination requirements from Section 3 can be rearranged to isolate scene-illumination, yielding

$$
\mathrm{I}_{\mathrm{S}}=\frac{4 \mathrm{I}_{\mathrm{F}^{\mathrm{f}}}}{\mathrm{RT}_{\mathrm{L}}}
$$

where:

$I_{8}=$ scene illumination

$I_{F}=$ faceplate illumination

f $\quad=$ f-number of lens

$\mathbf{R}=$ scene reflectance

$T_{1}=$ lens transmissivity 
Table 4-1. Lamp characteristice

\begin{tabular}{|c|c|c|c|c|c|c|}
\hline & Incandescent & $\begin{array}{l}\text { Quartz } \\
\text { Iodine }\end{array}$ & $\begin{array}{l}\text { Mercury } \\
\text { Vapor }\end{array}$ & $\begin{array}{l}\text { Motal } \\
\text { Halide }\end{array}$ & $\begin{array}{l}\text { High } \\
\text { Pressure } \\
\text { Sodium }\end{array}$ & $\begin{array}{l}\text { Low } \\
\text { Preasure } \\
\text { Sodium }\end{array}$ \\
\hline $\begin{array}{l}\text { Efficiency in } \\
\text { lumen/watt }\end{array}$ & $12-20$ & $20-23$ & $40-66$ & 80.100 & $95-130$ & $131-183$ \\
\hline Spectrum & $\begin{array}{l}\text { Broad, High } \\
\text { in Infrared }\end{array}$ & $\begin{array}{l}\text { Broad, High } \\
\text { in Infrared }\end{array}$ & Blue Green & Broad & Gold-Yellow & $\begin{array}{l}\text { Mono- } \\
\text { chromatic } \\
\text { Yellow }\end{array}$ \\
\hline Restrike Time & Immediate & Immediate & $\begin{array}{c}\text { 3-7 } \\
\text { Minutes }\end{array}$ & $\begin{array}{c}10-20 \\
\text { Minutes }\end{array}$ & $\begin{array}{l}2 \text { Minutes } \\
\text { or leses }\end{array}$ & $\begin{array}{c}\text { Immediate } \\
\text { to } 2 \\
\text { Minutes }\end{array}$ \\
\hline $\begin{array}{l}\text { Time of Full } \\
\text { Output (initial } \\
\text { or restrike) }\end{array}$ & Immediate & Immediate & $\begin{array}{c}3-7 \\
\text { Minutes }\end{array}$ & $\begin{array}{c}3-5 \\
\text { Minutes }\end{array}$ & $\begin{array}{c}3-4 \\
\text { Minutes }\end{array}$ & $\begin{array}{l}\text { 8-15 Minutes } \\
\text { (initial) } \\
5 \text { Minutes } \\
\text { (restrike) }\end{array}$ \\
\hline $\begin{array}{l}\text { Lamp Life } \\
\text { in hours } \\
\text { (lamp size) }\end{array}$ & $\begin{array}{l}750 \text { to } 10,000 \\
(100 \mathrm{~W})\end{array}$ & $\begin{array}{c}2,000 \\
(1,500 W)\end{array}$ & $\begin{array}{c}24,000 \\
(100-400 \mathrm{~W})\end{array}$ & $\begin{array}{r}15,000 \\
(400 \mathrm{~W})\end{array}$ & $\begin{array}{r}20,000 \\
(400 \mathrm{~W})\end{array}$ & $\begin{array}{r}18,000 \\
(180 \mathrm{~W})\end{array}$ \\
\hline $\begin{array}{l}\text { Voltages } \\
\text { Available }\end{array}$ & $5-5,000$ & $45-5,000$ & $175-3,000$ & $175-1,500$ & $50-1,000$ & $35-180$ \\
\hline
\end{tabular}

For example, assume the following representative values:

$$
\begin{aligned}
& I_{\mathbf{F}}=0.02 \mathrm{fc} \text { (average silicon array camera) } \\
& \mathbf{f}=1.4 \text { (fast lens) } \\
& \mathbf{R}=0.25 \text { (graded and smoothed ground) } \\
& \mathrm{T}_{\mathbf{L}}=0.8
\end{aligned}
$$

For this particular camera configuration, the required scene illumination is then $0.8 \mathrm{fc}$ to provide sufficient illumination for good low light level camera resolution. Note that scene reflectance varies with environmental factors: normally being minimum when rain wettened and maximum when snow covered. The NRC requirement for exterior lighting is $0.2 \mathrm{fc}$ (minimum) measured horizontally at ground level. The user should verify that the lighting level will provide usable video for the camera scene. 
(2) Vertical illumination-Light striking a vertical plane is termed vertical illumination. This light should be intense enough to provide good contrast between an object in the field and the background. The required level of lighting for this is difficult to estimate; however, for most exterior lighting systems satisfying horizontal illumination requirements, vertical illumination is at an adequate level.

(3) Light/dark ratio-Equal in importance to light levels, but often not considered, is the ratio of illumination levels between the brightest and darkest areas in the scene. The camera electronics average the scene illumination to set the iris control and AGC level. If the light/dark ratio is excessive, light areas will provide too much light, causing blooming or wash-out of details. Likewise, the dark areas will not provide sufficient light for good resolution.

A light/dark ratio of six to one should be considered to be the maximum, while a ratio of less than four to one is strongly suggested for exterior lighting. This includes the entire area observed by the camera and not just the area of interest. That is, lighting should extend beyond corners and fences to provide even illumination over three-fourths of the camera field of view. The area between the fences should be uniformly light-colored with a minimum reflectance of $\mathbf{3 0}$ percent when dry.

(4) Lighting spectrum-Camera imagers are like the human eye in that they are sensitive only to a limited band of the electromagnetic spectrum. If the light output spectrum and the camera imager sensitivity spectrum are different, more light may be required to provide adequate illumination. The lighting spectrum may not correspond to the area of maximum imager sensitivity, so a higher light level will be required than for broad spectrum illumination.

If an infrared energy source were used, a silicon array or solid-state imager equipped camera could function even if no illumination were visible to the eye. Since most lighting applications deal with visible light, errors can be made in estimating the light required if the camera imager spectrum is not considered. Figure 4-1 illustrates the spectral response of various imagers. The 589 line indicates the main spectral emission of sodium vapor luminaires.

\subsection{Design}

After lighting requirements have been determined, manufacturers and suppliers of lighting equipment should be consulted. Most major lighting suppliers have access to computer programs that can model a proposed lighting system to provide expected horizontal and vertical illumination levels over the area of interest. Maximum and minimum levels and light/dark ratio can also be determined. With access to such a program, a number of possible lighting configurations can be evaluated. The inputs to a computer program normally include specification of light fixture and lamp, mounting height, light pole spacing, and fixture orientation. The light output, as a function of angle from the fixture, also needs to be programmed. These data, however, are provided by the lighting manufacturers and are normally incorporated in the lighting suppliers' programs. If extensive lighting work is to be performed by a particular facility, a lighting program for in-house use should be considered.

The lighting configuration should be tested by installing a minimum of five fixtures (for single row installations) and actually measuring the illumination present. Measurements should be made at ground level at a sufficient number of equal intervals along straight-line parallel paths to locate the brightest and darkest points along the lines. Lighting patterns can also be estimated by using commercially available software that runs on personal computers.

Adjustment and modification of the total lighting system after installation should be anticipated. It is not uncommon to discover during camera testing that there are spurious reflections, bright spots, etc., that need to be corrected.

The respective horizontal and vertical illumination levels required for interior lighting are affected by the geometry of walls and obstructions. Reflections from room surfaces will also influence the lighting required. Major adjustment and/or modification of lights may be required to achieve satisfactory interior video performance. 


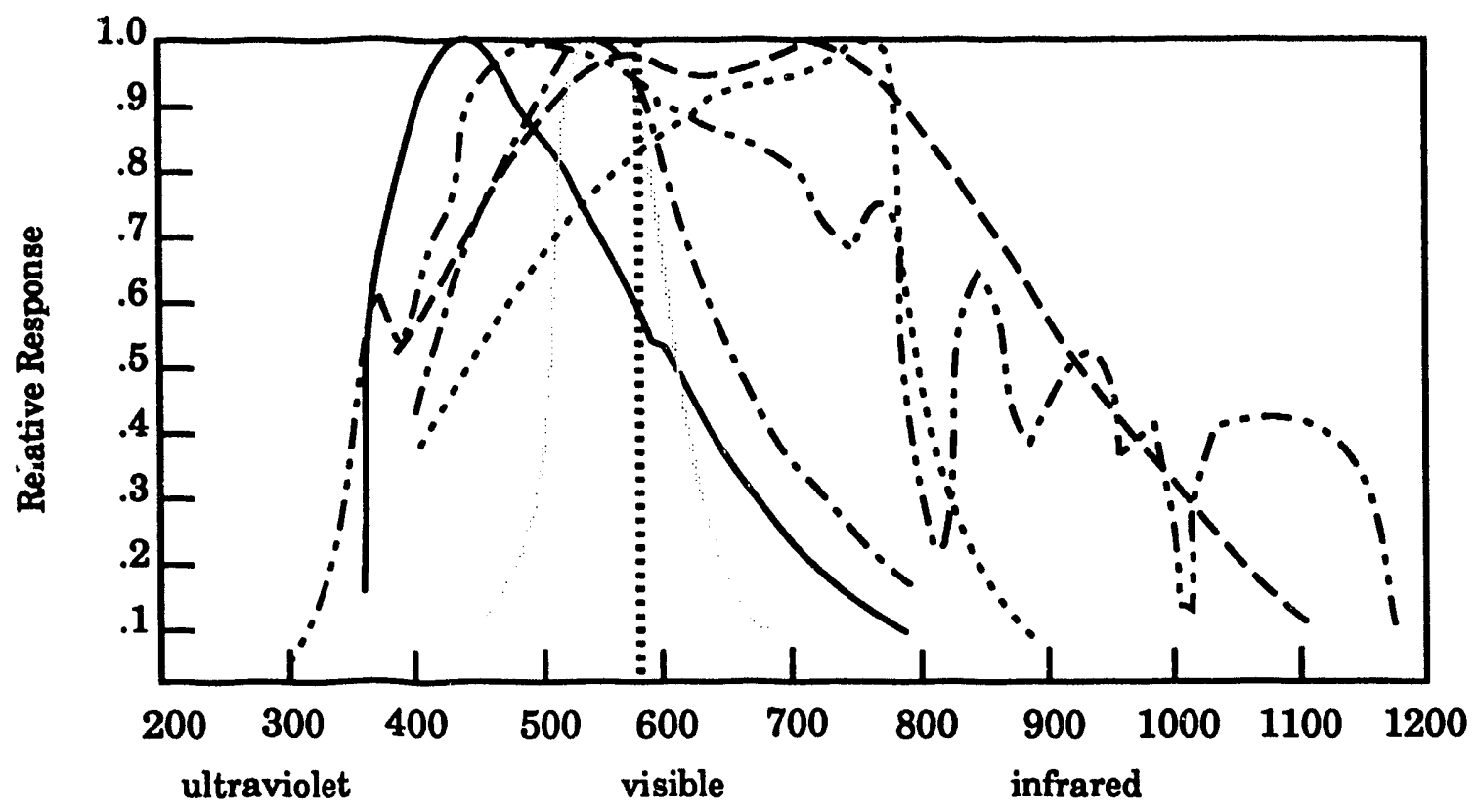

Wavelength (nanometers)

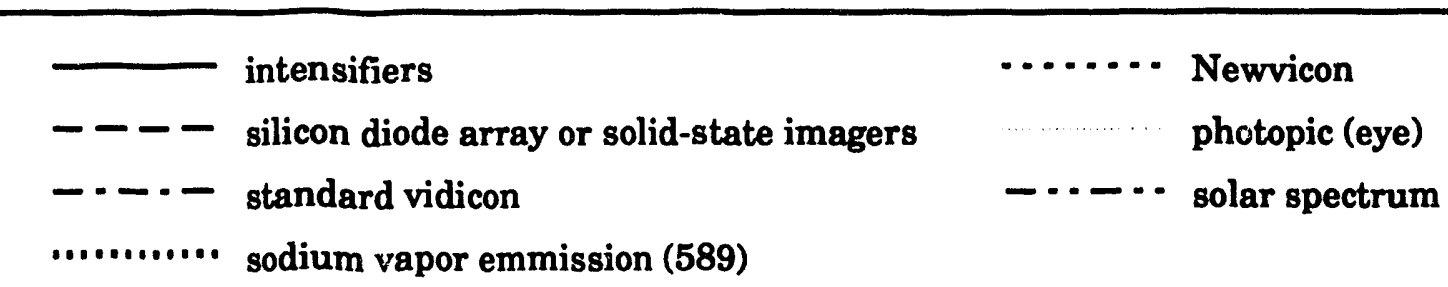

Figure 4-1. Spectral response

Any interior lighting level suitable for human comfort and safety, between 30 and $100 \mathrm{fc}$, is sufficient for the less expensive cameras. This assumes that surveillance and/or assessment is not required in a darkened room. If a constant light level is provided and maintained for all assessment needs, the manual iris lenses can be used with some cost-saving pussibility. 


\section{VIDEO TRANSMISSION SYSTEM}

When the video assessment cameras are located a significant distance from the video monitors, a transmission system is required to carry the video signal between these points. The signal at the end of the transmission path should be as faithful a reproduction of the original signal as possible. Any technique for transmitting a video signal results in some signal degradation, but proper selection of a transmission mode, tailored to the site-specific conditions, can result in a cost-effective, technically acceptable system.

\subsection{Cable}

The most common method of video transmission is by cable, which has many forms. Coaxial cable is the most common choice, although twisted pair, triaxial, and twinaxial (balanced line) systems are sometimes selected for their specific characteristics. Video signals are normally transmitted from 75ohm signal drivers that, for most efficient use, should be carried on 75-ohm cable and terminated at the end of the line with a 75-ohm characteristic impedance.

The performance required of a video cable is based upon the frequency requirements of the video signal to be transmitted through it and the physical and/or electrical environment in which it will be used. Any transmission line has resistive and reactive losses. These losses, primarily functions of the distributed capacitance and conductor resistance, are usually expressed in decibels (dB) per unit, length. Signal attenuation increases with both the signal frequency and cable length and varies widely among the various cable types.

Several core/shield combinations are common in video cable. The differences are largely due to the relative ability of any given configuration to operate in adverse electromagnetic environments without significant signal degradation. The most comrc on configurations for video transmission are coaxial and triaxial 75-ohm cables and twinaxial 124-ohm cables. Figure 5-1 illustrates cross-sections for these configurations.

Differences exist in the composition of the outer insulating jacket, depending on the ultimate use of the cable. Exterior cable is usually insulated with polyethylene suitable for direct burial or continuous exposure to weather. Armoring is accomplished by wrapping a polyethylene-covered steel sheath around the cable. Overhead cabling in which an integral messenger wire is incorporated is also available.

For installations where electromagnetic interference (EMI) is prevalent, the interference phase cancellation properties of balanced line twinaxial cable or the additional shielding capabilities of triaxial cable may be desirable. Twinaxiál cable for CCTV systemis is $124 \mathrm{ohms}$ (nominal) and will generally require impedarice matching transformers or amplifiers. If properly used, matching or signal isolation transformers will also eliminate alternating current $(\mathrm{AC})$ ground currents in the paths of coaxial and triaxial cable runs.

Assurning that correct impedance matching is maintained, the attenuation characteristics are readily available from the cable manufacturer. Table 5-1 lists the characteristics of some of the common types of cable.

For a given distance and frequency, the cable attenuation in $\mathrm{dB}$ can be determined. If cable loss is greater than about $6 \mathrm{~dB}$ at the highest frequency, some form of signal conditioning may be required to maintain optimum performance. The decision on whether to buy signal conditioning should be dependent upon the actual system resolution requirements and should be considered in light of the additional expense of the conditioning equipment and for recurring maintenance.

The most common coaxial cable used is the RG-59 type. It can be used in all short lengths typically found in a security center. Although this cable produces considerable losses, its small diameter, low cost, and flexibility make it popular. The RG-59 cable can be used for camera-to-monitor lengths up to a few hunured feet, if netesssañy, without appreciable signal degradation or the necessity for signal conditioning equipment. Coaxial cable of smaller diameter, such as RG-179 or RG-187, should be used in the 
Figure A

Coaxial Cable

Cross-Section

(75-ohm impedance)

Figure B

Triaxial Cable

Cross-Section

(75-ohm impedance)

Figure C

Twinaxial Cable

Cross-Section

(124-ohm impedance)

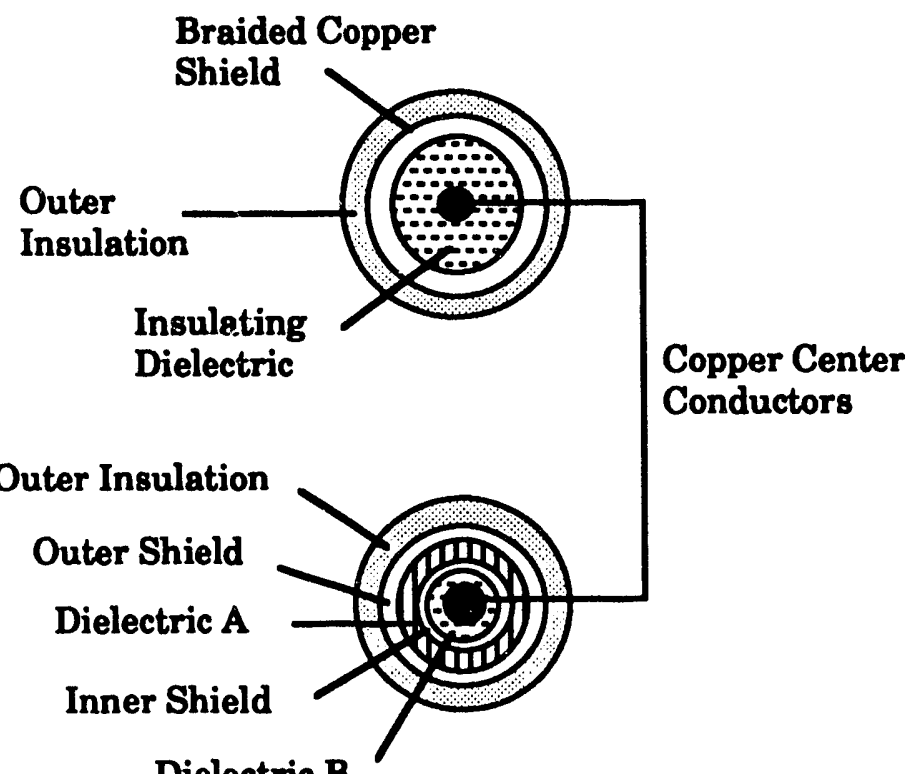

Dielectric B

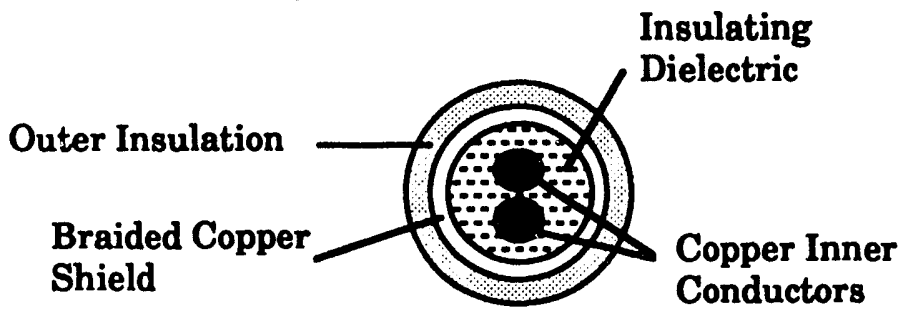

Figure 5-1. Cross-8ections of typical video cables

shortest lengths possible and only if bulkier cable is too large, as in video switcher input and output panels. These miniature cables have about five times the high frequency loss per unit length of the RG59 cable. RG-59 coaxial cable is frequently used in camera control cables having power, video, remote camera control functions, and lens control functions in a single, multiconductor cable assembly.

Larger diameter cable such as RG-11 is commonly used when the camera video runs are several hundred feet. RG-11 has about 40 percent of the high frequency loss per unit length of RG-59 cable. This type of cable can be selected with an insulating jacket suitable for direct burial if the presence of small wildlife does not present a hazard to the insulating jacket.

A third type of coaxial cable, the semi-rigid, aluminum-shielded cable, is used at video baseband and radio-frequency (RF) modulated frequencies. Cable running hundreds of feet can be accommodated without signal conditioning at video baseband frequencies. This type of cable is used by the cable TV industry and for security applications where multiple channel transmission is more efficient than multiple runs of baseband video coaxial cable. It has high frequency losses per unit length of about onehalf to one-third of those in RG-11 cable, depending on the cable diameter.

Aluminum clad cable is the most expensive cable discussed, but in some installations, it could offset the cost if signal conditioning equipment became unnecessary. The standard signal conditioning hardware for cable TV transmission allows only about $4 \mathrm{MHz}$ of video bandwidth in its standard configuration. However, equipment manufacturers have provided modified double-bandwidth equipment at a modest cost increase to provide a more acceptable frequency response for security applications. 
Table 5-1. Video cable characteristics

\begin{tabular}{|c|c|c|c|}
\hline $\begin{array}{c}\text { Cable } \\
\text { type }\end{array}$ & $\begin{array}{c}\text { Impedance } \\
\text { ohms }\end{array}$ & $\begin{array}{c}\text { Jacket OD } \\
\text { in (cm) }\end{array}$ & $\begin{array}{c}\text { Attenuation at } \\
10 \mathrm{MHz}, \mathrm{dB} / 100 \mathrm{ft}\end{array}$ \\
\hline $\begin{array}{c}\text { RG-59/U } \\
\text { coaxial }\end{array}$ & 75 & $0.242(0.615)$ & 1.10 \\
\hline $\begin{array}{c}\text { RG-59/U } \\
\text { triaxial }\end{array}$ & 75 & $0.315(0.800)$ & 0.73 \\
\hline $\begin{array}{c}\text { RG-11/U } \\
\text { coaxial }\end{array}$ & 75 & $0.405(1.029)$ & 0.66 \\
\hline $\begin{array}{c}\text { RG-11/U } \\
\text { triaxial }\end{array}$ & 75 & $0.475(1.207)$ & 0.40 \\
\hline $\begin{array}{c}\text { JT 4412 } \\
\text { Semi-rigid coaxial }\end{array}$ & 75 & $0.412(1.046)$ & 0.21 \\
\hline $\begin{array}{c}\text { JT 4500 } \\
\text { Semi-rigid coaxial }\end{array}$ & 75 & $0.500(1.27)$ & 1.70 \\
\hline $\begin{array}{c}2092 \\
\text { Twinaxial }\end{array}$ & 124 & $0.242(0.615)$ & 0.70 \\
\hline $\begin{array}{c}9860 \\
\text { Twinaxial }\end{array}$ & 124 & $0.440(1.118)$ & 0.26 \\
\hline
\end{tabular}

Aluminum clad cable is more difficult to handle because of its aluminum shield, minimum bend radius, and susceptibility to decreased performance from damage in mishandling. If semi-rigid coaxial cable is selected, it should be of the armored variety. Armoring provides some protection from rough handling. Even armored cable is susceptible to damage by kinking or denting with a resulting impedance mismatch and signal degradation. Many contractors are not familiar with installation of semi-rigid coaxial cable and may require training in handling procedures.

Once the length of the cable running between the camera and the monitor has been determined, the cost of each cable type and its installation cost should be considered together. In some cases, the cable length may be such that signal conditioning may be required regardless of the type of cable selected. In such a circumstance, a less expensive or more easily handled cable can be selected if the cost of the signal conditioning is not offset by the choice of the less costly cable.

The design of the cable transmission system should consider the bandwidth of the overall video system including the camera and the monitor. For the most cost-effective solution, all the elements of the system should be of comparable resolution capability. Transmission systems can be tailored to provide almost any bandwidth. High-quality monitors usually provide high resolution. The primary bandwidth limitation is the camera whose imager and format will determine the live image quality. The transmission system should not degrade the camera bandwidth so as to nullify the selection of a high resolution camera. If significant bandwidth degradation seems inevitable under the site specific restrictions, then signal conditioning equipment should be considered.

In determining the bandwidth and resolution requirements, a common rule of thumb states that $1 \mathrm{MHz}$ of bandwidth is equivalent to a spatial resolution of $80 \mathrm{TV}$ lines. For example, a 10-MHz bandwidth can resolve 800 TV lines of resolution. 
Before any installation is begun, a test of the selected components should be made over the maximum expected length of cable run in order to evaluate the choice of cable and equipment. System design from manufacturers' specifications alone may not always provide the system results intended.

For underground cable installation, time-domain reflectometer (TDR) measurements should be made after the cable has been laid and all necessary splices have been made, but before the cable is buried. These measurements should detect any cable breakage or impedance anomalies. Trenches should be deep enough to preclude cutting cables during routine surface maintenance such as smoothing, grading, and cleaning of drainage ditches. For above-ground installation, cable routing should avoid potential sources of noise and inadvertent damage. Cable runs in conduit can reduce the possibility of damage and deter tampering.

All cable connectors, splices, feedthroughs, barrier strips, crimp terminals, clamps, and interface panels should be specified by drawing to identify installation and performance requirement. These components should not require a high degree of skill for installation, since much of this work neea to be performed in the field.

\subsection{Connectors}

A large variety of connector types and styles is available for connecting video equipment and cables. Selection of connector type is usually determined by the type of cable in use and by the type of connectors supplied on the equipment. Crimp and solder connections are available for most types of connectors. Both sonnections are acceptable, but crimp connections may be easier in field installations.

The primary types of connectors for the cables described are the BNC, twinaxial, UHF, and Type F. Any connector selected for video applications should have a characteristic impedance matching that of the cable, typically $75 \mathrm{ohms}$ for unbalanced cable and $124 \mathrm{ohms}$ for balanced cable. These connectors are illustrated in Figure 5-2.
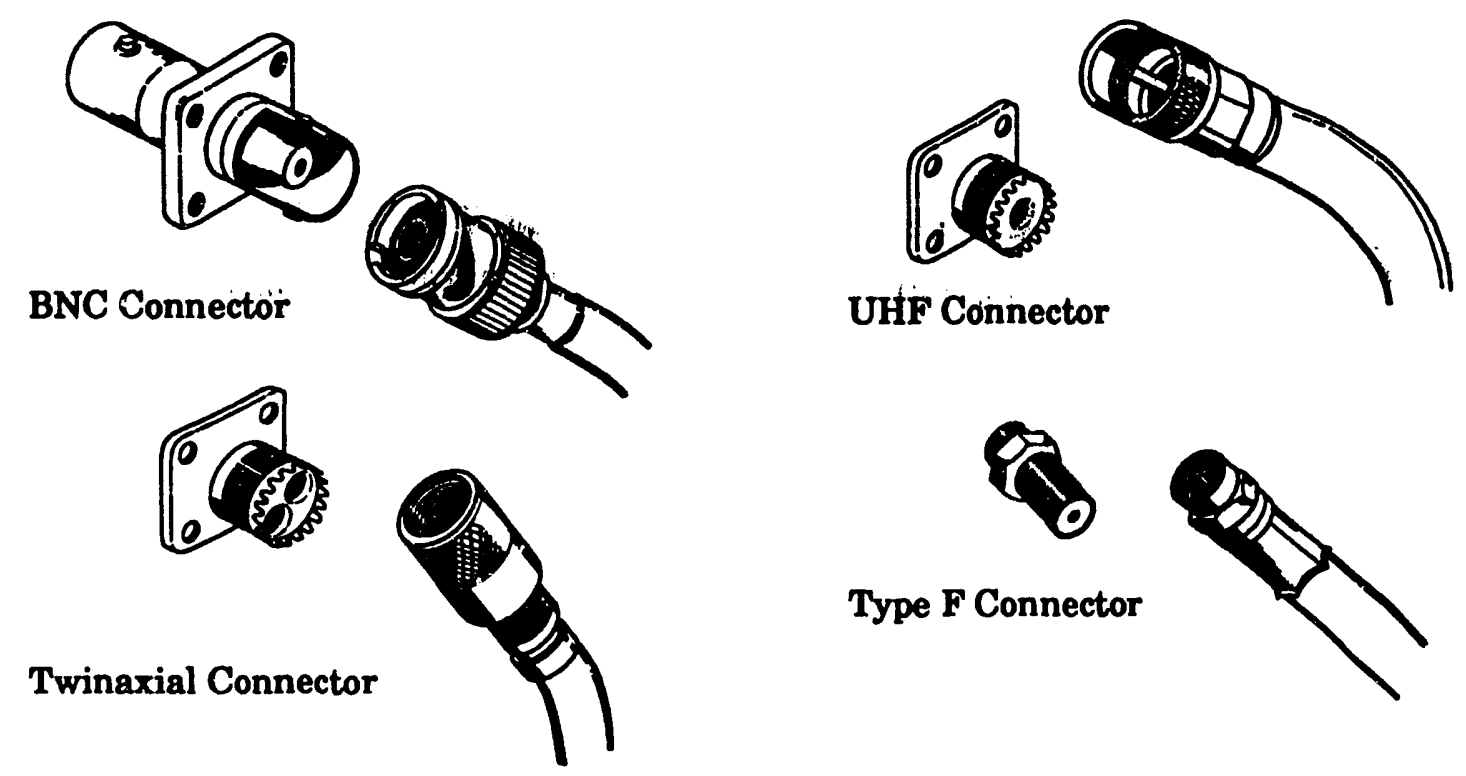

Figure 5-2. Cable connectors 


\subsection{Signal Conditioning}

Any video cable will cause some degradation to the video signal. High-frequency attenuation, induced noise, and phase shifts, can radically alter the amount of useful information in the video signal. Highfrequency attenuation reduces picture detail (loss of resolution), while phase shifting can cause image smear or "ghosts" because of impedance discontinuities along the cable or at splices and cable connections. Induced noise can be seen on the monitor as high-frequency or random "snow," or as power line noise (light and dark horizontal bars on the monitor). Both forms of noise can partially obscure the desired image on the monitor, effectively reducing system resolution.

To compensate for these signal degradations, signal conditioning should be performed at one or more points along the video transmission path. A wide range of video conditioning equipment is available commercially.

\subsubsection{Video Equạ!izers}

Video equalizers compensate for the high-frequency signal losses in long lengths of video cable. The amount of equalization needed depends on the cable selected and its length. Equalizers usually provide a gain adjustment to compensate for resistive losses in the cable and one or more high frequency adjustments. Some equalizers may contain 12 to 15 adjustments to compensate selectively for losses at different frequencies. For most situations, an equalizer with fewer adjustments may be adequate if signal quality is not seriously diminished. A test signal generator and a multiburst or sweep frequency generator can determine the amount of loss that is tolerable with the required cable length. While manufacturers' specifications provide a guide in the selection of equalizers, it is preferable to evaluate several types of equalizers before final selection is made.

Equalization can be performed at either end of the transmission line. At the camera end, this technique is called pre-equalization and may be a fixed amount of selective amplification of the higher frequencies. This approach provides amplification before the signal amplitude is reduced by the cable run. When used at the receiving end, the technique is called post-equalization. Post-equalization offers easy adjustment, since this end of the cable is usually located where test equipment can be easily accessed and the effects of equalization on signal quality easily determined. Post-equalization does have the disadvantage that in amplifying the signal some noise will be added to the signal. For exceptionally long cable runs, both pre- and post-equalization can be used.

\subsubsection{Hum Clampers}

A common problem in long video cable runs is that of power line frequency signals being combined with the video signal. This occurs because grounds in separate locations are often not at the same potential. These potential differences at the ends of the cables cause power line frequency currents to flow in the outer shield in unbalanced (75 ohm) lines. The signal path is shown in Figure 5-3.

If this signal is of sufficient amplitude, the effect may be seen on the video monitor as hum bars, or horizontal light and dark bars in the video signal. The signals can be reduced or eliminated by devices commonly known as clampers, hum eliminators, or clamping amplifiers or by differential input amplifiers that isolate the video signal from a ground potential.

The transformer is the simplest form of hum eliminator, which passively acts to isolate the video cable shield from ground potential. This effectively breaks the power line frequency signal path, eliminating the current flow. Figure 5-4 illustrates this effect.

The active hum clamper may use the power line signal in an out-of-phase condition and add it to the video signal. This results in the cancellation of the power line frequency signal and leaves a clean video signal without the induced hum. Other types of clampers use the blanking level of the incoming composite video signal as a reference and clamp that level with reference to a fixed potential. 


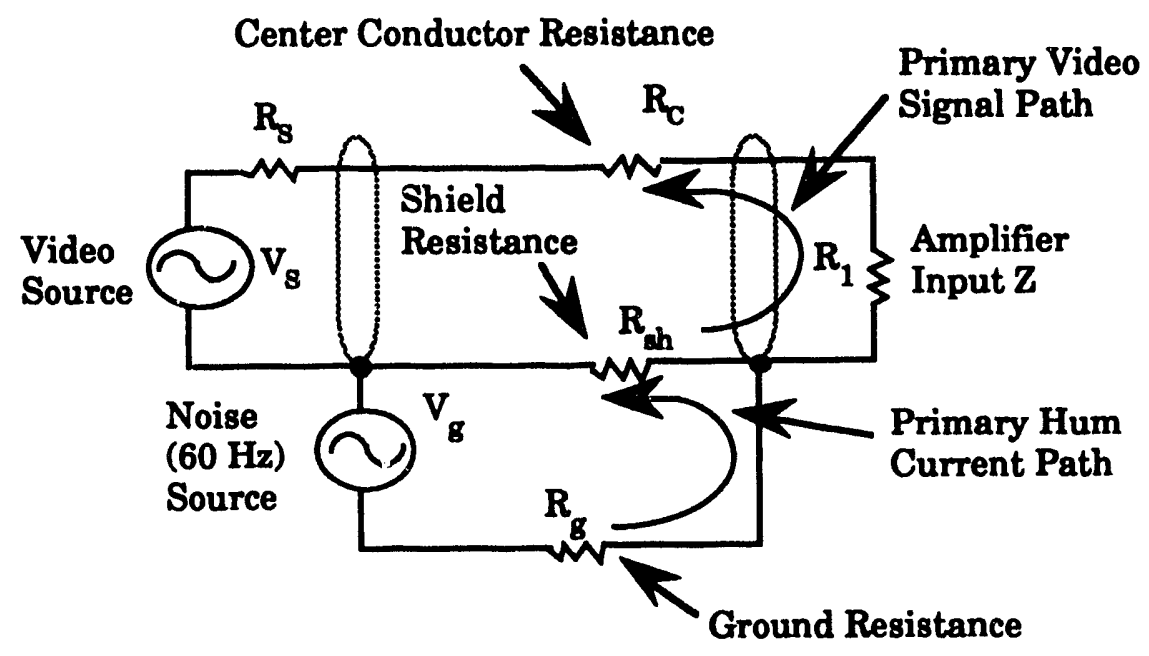

Figure 5-3. Hum induced into video line

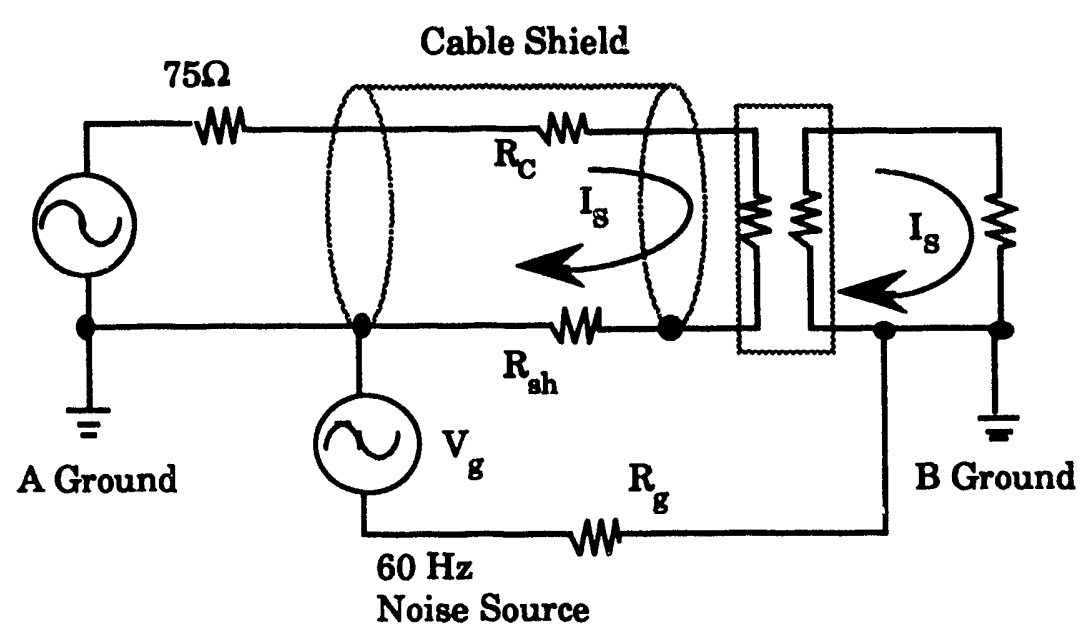

Figure 5-4. Hum cancellation by isolation transformer

In a balanced line transmission system, any noise induced in the lines is coupled to both sides of the line at the same time, thus nullifying their effects. These balanced line systems, usually of 124-ohm impedance, require a matching transformer or amplifier to transform the impedance of the video line from $75 \mathrm{ohms}$ to $124 \mathrm{ohms}$ and back again at the other end. Figure 5-5 illustrates this type of system.

\subsection{Fiber-Optic Transmission Systems}

Fiber-optic transmission systems use an optical path rather than an electrical path for the transmission of information. The conductor is a glass fiber rather than copper. An electro-optical transmitter is required at the sending end of the optical fiber to convert the electrical video signal into an optical signal. This can be done by a simple light-emitting diode (LED) or a high-intensity laser. 


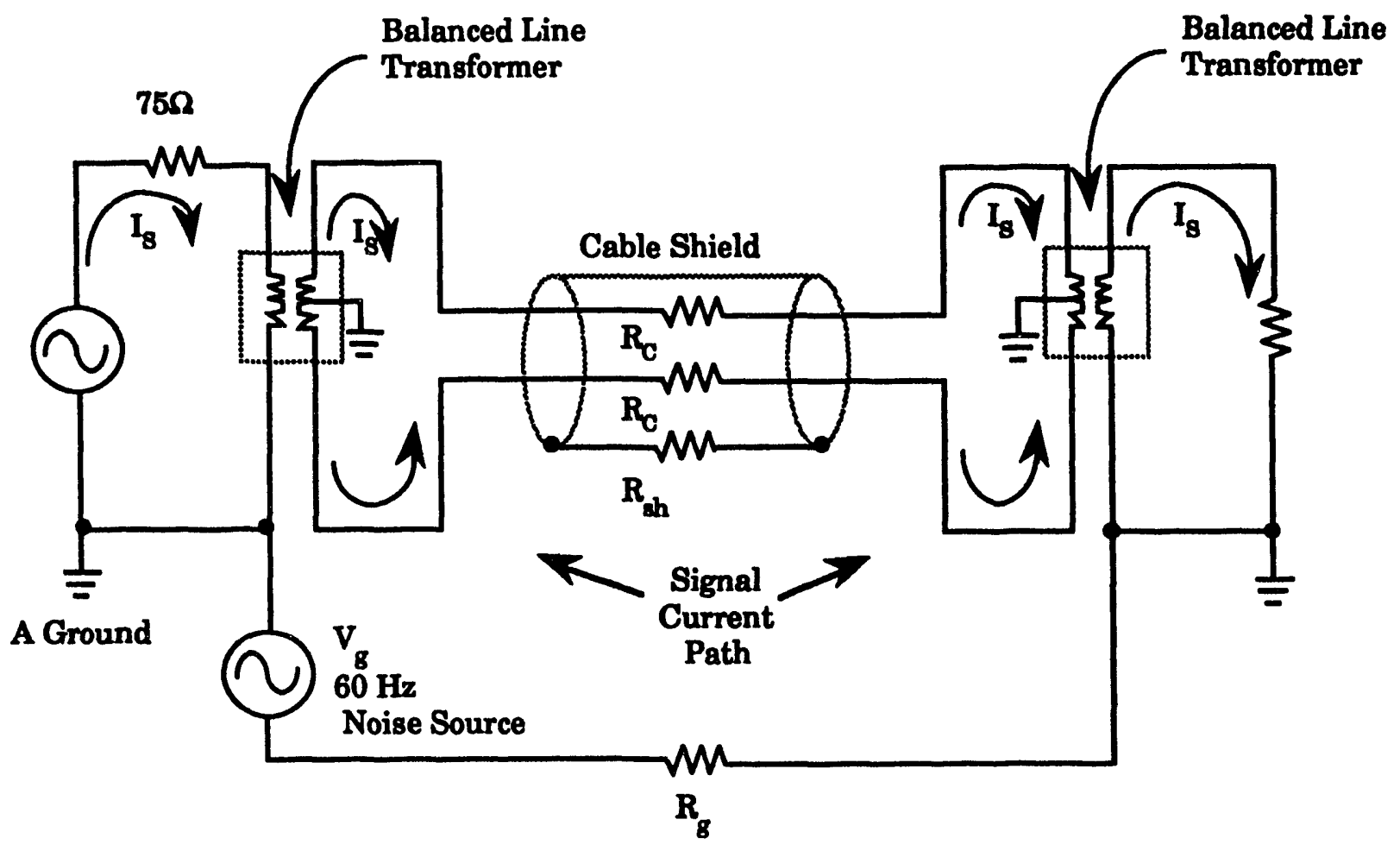

Figure 5-5. Hum cancellation by balanced line

The maximum usable length of the fiber transmission path is affected by the source power and wavelength; the fiber diameter and material; the connector and splice losses; and the receiver gain. In the electro-optical receiver, a photodiode converts the optical signal back into the electrical video signal present at the camera.

The advantages of fiber-optic transmission over copper wire are

- immunity from induced noise

- no radiated EMI or radio-frequency interference (RFI)

- immunity from external EMI and RFI

- no ground-loop electrical path

- reduced susceptibility to equipment damage from lightning

- wider bandwidth capability

The glass-fiber optical conductors used for video transmission are low-loss silica-based fibers. The core is the primary optical path and is surrounded by cladding to contain the light energy within the core. The cladding should have a lower index of refraction to reflect the light energy back into the core. The index of refraction is the ratio of the velocity of light in a vacuum to the velocity of light through a given optical medium. As the density of a light-conducting material increases, the velocity of light decreases, resulting in an increase in the index of refraction. The index of refraction is greater for shorter wavelengths of light, which means that they propagate more slowly along the optical fiber.

In very small diameter (4-12 microns) fibers, the light from the source is confined primarily along the axis of the fiber. This propagation method is defined as single mode since the propagation is along a single path. Since tine fiber is small, it can accept incoming light only over a very small acceptance angle, meaning that less light from the source is coupled into the fiber. The acceptance angle can be defined by 
the numerical aperture (NA), which relates to the indices of refraction between the core and the cladding. Larger numerical apertures mean higher acceptance angles.

Single mode fibers can conduct wide bandwidth signals over long distances. The bandwidth and the length of the fibers determine the amount of signal attenuation. Fiber cable attenuation for single mode fibers is typically less than $1 \mathrm{~dB}$ per kilometer of length. Light sources for this type of transmission are usually laser diodes, particularly for longer distance, wider bandwidth applications. The operating wavelengths for these sources are in the near-infrared region of the spectrum, typically at 1.3 and 1.5 microns. The receivers are usually avalanche photodiodes that intrinsically have some amplification, although they are more costly and require more power than PIN photodiodes.

As fiber diameters increase, the acceptance angles increase, resulting in more light from the source being coupled into the fiber. The light enters the fiber at many different angles and is propagated through the fiber along many different paths. This is defined as multimode propagation. Multimode fibers can be found in diameters of 50 to 1,000 microns. The smaller diameter fibers are made of glass, while the larger diameter fibers are plastic composition. Because of the high losses in plastic fibers, their use is limited to very short transmission distances.

The multimode propagation results in dispersion of the light as it passes along the fiber, resulting in a distorted signal at the end of the fiber due to the multiple light paths combining to form the output signal. By building the fiber with glasses of decreasing indices of refraction from the core outward, the longer light path signals will be propagater faster through the outer edges of the fiber. This reduces the light dispersion, resulting in a less distorted output signal. This type of fiber is called a graded index fiber.

Multimode fibers are usually used for short-distance transmission systems. The usable transmission length is reduced by the modal dispersion of the light signal. Fiber cable losses are typically on the order of 2 to $10 \mathrm{~dB}$ per kilometer of cable. Light emitting diodes are commonly used for the light source. The operating wavelength of these sources is in the range of 820 to 880 nanometers or near 1.3 microns.

In either type of fiber-optic transmission system, the number of connectors, splices, and taps will reduce the effective length of the system since there are light losses at all of these junctions. Each end of the fiber should be perpendicular to the fiber axis, and the end face should be as flat as possible to minimize losses. Connective losses can result from lateral misalignment, angular misalignment, and the gap between the fiber ends. Lateral placement is more critical for smaller fibers, while gap losses increase with the size of the gap and are greater in high numerical aperture fibers.

Connectors are more difficult to terminate on single mode fibers. Typical losses are on the order of 0.2 $\mathrm{dB}$. Multimode connectors are less expensive but have losses in the $0.3-$ to $0.5-\mathrm{dB}$ range. Special tooling and expertise are frequently required to terminate fibers.

There are several common types of connectors in use for the various fiber types. The type of connector to be used usually will be determined by the manufacturer of the transmitter and receiver selected. The manufacturers' recommendations in selecting the fiber and connector type should be considered.

Splices can be made in fiber cable at lower losses than connectors, provided care is exercised. For very low loss splices, as low as $0.1 \mathrm{~dB}$, some special hardware and/or tooling is required. In some instances, special bonding adhesives are used that have an index of refraction matched closely to that of the fiber.

One difficulty with signal distribution by fiber optics is that switching and loop-through configurations result in a considerable loss in signal strength. Signal restoration by active elements can compensate for these losses, but active elements can fail, reducing system reliability. These switching and distribution functions may be better served by electronic means for the foreseeable future. 
Multiconductor bundles of fiber-optic cable can be combined in a protective jacket for a variety of environments. The cable can have metallic or nonmetallic strengthening members to allow the cable to be pulled in conduit much as electrical cable. Care should be exercised in the handling, installation, and termination of the cable to avoid fracturing the glass fibers in the cable. The minimum bend radius of the individual fibers should be observed when routing and terminating the fibers.

At this time, fiber-optic cable is more expensive than coaxial cable, but there are situations where fiberoptic cable may be the better choice for the distance or for the environment. Some manufacturers have packaged single-fiber video transmission systems with transmitter, receiver, and terminated cable in lengths exceeding a kilometer. For exterior video assessment transmission systems, rack-mounted, multiple-module receivers are available.

In designing or specifying a fiber-optic transmission system, the basic system requirements should first be established for bandwidth and distance. The bandwidth should be sufficient to preclude a reduction in resolution from the camera. The transmitters and receivers, frequently sold in pairs for specific applications, can then be considered. The transmitter/receiver pair should have sufficient power and sensitivity to function reliably over the required distance through a selected fiber. The type and size of the fiber and the connector type will usually be determined by the manufacturer of the transmitter and the receiver. Some manufacturers offer user-specified connector options. The total system optical loss (loss budget) should be less, by a comfortable margin, than the transmitter power (power budget). This loss budget is the sum of the individual losses attributable to the fiber and connectors and the power required by the receiver, as well as by the safety margin to allow for additional connectors, taps, or splices necessary at installation and for future maintenance requirements. The help of the transmitter/receiver manufacturers should be sought for determining the fiber selection and loss budget considerations for their particular equipment.

\subsection{Microwave Transmission Systems}

Microwave transmission is commonly used by the broadcast television industry to relay program material to transmitters from a remote site or studio facility. Equipment is commercially available for wider bandwidth transmission for security applications. The use of microwave transmission is complicated by the requirements for Federal Communications Commission (FCC) approval for frequency allocation. An obvious restriction is that any microwave-transmitted video signal can be intercepted along and beyond the signal path very easily without detection. While technically plausible, and in many cases extremely cost-effective, these systems have not been widely used in security applications.

\subsection{Optical Transmission Systems}

Optical transmission systems have much the same characteristics as microwave transmission systems, except that they do not require FCC approval for operation. The video signal is transmitted by amplitude modulation of an LED or laser. Typically an audio (or data) channel is provided.

As in microwave transmission systems, the chief advantage of optical transmission systems is the ability to provide line-of-sight transmission across or among physical obstacles without the need for laying cable. This can be especially useful in site security retrofit installations. Another advantage to optical transmission systems is their immunity to interference in high electromagnetic or electrostatic environments. Their maximum useful range may be only several hundred to a few thousand feet, but they can be very cost-effective in the right circumstances. Temporary transmission paths can be easily accomplished with optical transmission systems.

Their main disadvantage is that operation may be hindered in fog, rain, or snow, depending on the density and the distance between the transmitter and the receiver. 


\section{SYNCHRONIZING SYSTEMS}

In an ideal CCTV system, the scanning electron beam or the solid-state imager readout generating the video signal and the electron beam creating the image on the video monitor are at identical relative locations at identical times. Sync pulses control this time/position relationship by controlling the oscillators driving horizontal and vertical blanking and deflection circuits. The choice of synchronizing technique for an assessment system will be influenced by system size, system complexity, and the available budget. The choices are to use random local sync or line-lock sync in the camera or to provide a master sync source for the entire video system.

\subsection{Local Sync}

In the local sync option, oscillators in each camera generate the sync pulses that may differ from camera to camera in timing and frequency. Local sync is the easiest and least expensive way of providing system sync, but it also produces the least aesthetically pleasing monitor display. Vertical rolling may appear on the monitor briefly after switching between cameras. Video recorders may lose critical assessment information because of delay in sync lockup after switching between sources. In addition, local sync may not be compatible with some other video equipment such as character generators and video motion detectors.

\subsection{Line-Lock Sync}

Some camera sync generators can be locked to the AC power-line frequency. All line-locked cameras that derive power from the same AC power-line phase will have their sync pulses in phase with each other. In addition, some cameras have phase-shift circuitry that permits line-lock operation from any phase of a three-phase power system. In some circumstances, this line-lock mode may be sufficiently accurate to allow vertical interval switching without a master sync system. The total system should be considered before this option is acceptable. This operation should be verified before committing to a large equipment expenditure. There should be no other equipment that requires an extremely stable and repeatable sync input to function properly.

\subsection{Master Sync}

A master sync system provides horizontal and vertical synchronized scanning signals to drive CCTV cameras, video recorders, and other video equipment. There is nothing particularly unique about these sync generators as compared to their self-contained counterparts in video cameras, except that they are virtually always fully interlaced and possess excellent stability. Temperature controlled crystal ovens are frequently used to provide the necessary frequency accuracy. Sync generator options include separate horizontal and vertical drive signals, various test signals, and genlock (the ability to phase lock to an external source). Use of master sync should provide glitch-free switching, display, and recording. However, it usually requires a separate sync in put cable to each camera, which increases the total system cost. Some additional equipment can be used to supply sync signals to the camera and receive the video signal on a single coaxial cable.

\subsection{Sync Distribution}

While individual sync generators can drive several outputs, they cannot directly drive all the cameras, even in a small assessment system. The sync generators are coupled with pulse distribution amplifiers for a separate output to each camera. It should be noted that the cameras selected should be able to accept external sync. Sync distribution systems provide the means of supplying each device in the assessment system with the same sync signal. Pulse distribution amplifiers are commonly available in modular form, facilitating maintenance and system expansion. Options include pulse regeneration and pulse delay for timing the sync signals over varying lengths of transmission line from the sync generator to the device. 


\section{VIDEO SWITCHING SYSTEM}

Most alarm assessment systems use more cameras than monitors because too many monitors will overload the operator, and all camera sectors will seldom have to be assessed simultaneously. For this reason, video switching equipment can be utilized between a large number of camera inputs and a smaller number of monitors and/or recorders.

The simplest type of video switcher connects one of a number of camera video inputs onto a single output bus. An output bus is a single, 75-ohm source impedance video switcher output signal that is typically terminated in a video monitor or recording device. An output bus may contain two video output connectors, but both will have identical video signals.

Only one monitor or recorder input can be connected to a single output bus at a time. These switchers are usually packaged for a single output bus in a single rack frame. Several rack frames can be grouped together for a greater number of output buses.

\subsection{Passive Switching}

Switching can be either passive or active. In a passive switcher, switching is by physical manual selection. The actual switching function is performed by push-button contacts, and the video signal is routed through the switch contacts with no electronic signal conditioning. A mechanical interlocking mechanism prevents more than one input from being connected to the output bus. Passive switching is useful primarily in systems having a limited number of cameras with no requirement for immediate assessment. Its chief advantage is its low cost.

\subsection{Active Switching}

Active switchers usually include input and output amplifiers to provide signal isolation, impedance matching, and video signal amplitude control. Electronic processing of the video signals can be included to control the timing of the switching between video signal inputs. The switching action occurs at mechanical switch contacts or a semiconductor junction. Active switchers are available as single output devices, or the individual units can be grouped (and mechanically connected) to form larger switcher matrices.

\subsection{Local/Remote Switching}

Video switshers can be controlled locally or remotely. The distinction is made at the point at which the switching function actually takes place. If switching takes place at the switcher control panel by direct action, then it is termed local switching. If switching occurs at a point away from the direct physical contact, then it is termed remote switching. Remote switching can occur at a distance of 1 meter or several hundred meters. All switchers can be remotely operated except manual switchers using push-button contacts for the switching function. Remote switching has two primary advantages. First, it frees equipment space in the operator's console. The bulk of the video switching units can be located in equipment racks away from the operator's console with only a small switcher control panel in the console. Large matrix switchers of 100 inputs or more can occupy several equipment rack frames.

Second, remote switching allows spatial separation of the parts of the switcher. This may be useful in eliminating long video transmission runs between cameras and monitors. For example, if 20 cameras are installed but only 4 monitors will be viewed at one time, part of the switcher can be close to the cameras, thus eliminating 16 cable runs.

\subsection{Gap Switching}

In gap switching, the first video input is removed from the output bus and terminated prior to connecting the second input onto the output bus. No signal is present on the output bus during the switching interval. This total loss of signal, if long enough, may result in monitors and recorders losing sync, briefly causing 
picture breakup or instability. The simplest, least expensive manual switchers are gap switchers. Switching typically takes 100 to 200 milliseconds.

\subsection{Lap Switching}

In lap switching, two video input signals are present on the output bus for the duration of the switching action. The presence of the two signals on the output bus may cause the image on the monitor to jitter during this transition time. The switching transient may last 100 to 200 milliseconds.

\subsection{Vertical Interval Switching}

If switching is always timed to occur during the vertical blanking interval, no picture discontinuities can exist since the switching is accomplished when the monitor screen is blank. Vertical interval switching requires synchronizing all incoming video signals. The common vertical sync is fed to the video switcher so that switching between sources occurs at the vertical blanking interval. This type of switching is more complicated and costly than gap or lap switching. However, it does result in a continuous, stable bus output signal that maintain monitor and recorder sync stability.

\subsection{Sequential Switching}

Sequential video switchers are used to view a multiple camera system for display or to record the individual video signals in sequence and repetitively. The sequential switching can be programmed for only the selected cameras since not all connected cameras need to be included in the camera sequence. The programming can include the dwell time (duration) that each video input will appear on the output bus. Dwell times are adjustable from 1 to 30 seconds. As the number of cameras in the scanning sequence increases, the dwell time should be reduced to minimize the interval between the time a particular image appears for the second and subsequent scan cycles. These switchers are most useful in surveillance applications.

For clean, glitch-free switching, the cameras should be in sync. With random sync inputs, the monitor display may jump or roll at each switching step in the scanning sequence.

Some sequential switchers are available with additional circuitry that will automatically switch to a selected output when a contact closure occurs at the switcher. The contact closure could come from a sensor associated with a particular camera field of view, instantaneously displaying the area around the activated sensor.

\subsection{Computer-Controlled Switching}

Computer-controlled switching provides automatic display of a video scene in which a sensor has alarmed, for the rapid assessment necessary in high threat situations. Although more expensive than other methods, computer-controlled vertical interval switching provides additional features that aid in the speed and accuracy of the assessment process and provide timely display and recording of significant events without operator intervention. The computer-controlled switcher can be programmed and interfaced to a video recording system to automatically record and playback the initially stored scene on a second monitor concurrently with the live video display. This is particularly important in multiple alarm situations where orderly management of a series of assessment functions should be performed quickly and as accurately as possible. The switcher controller can also be programmed for alarm prioritization and multiple sequential scanning patterns.

Any automatically controlled video switcher system should be provided with the means for manual control in various locations for operator selected surveillance, for initial system installation, and for routine maintenance and troubleshooting. 


\subsection{Switcher Selection}

The number of video inputs and outputs will determine the size of the switcher matrix. It should be remembered that inputs may include other than camera video sources, such as video recorder outputs and test signal generators, for monitor setup and maintenance. The number of output buses should include each video device in the video system including monitors, recorders, and possibly video line supervision equipmeni. Thus the system operating philosophy and all of the system components of the entire intrusion detection system should be established before the number of video inputs and outputs can be determined. The size of the video switcher is referred to by its input and output capacity, in that order. For example, a switcher having 40 inputs and 10 outputs is referred to as a 40 by 10 switcher.

Once the size of the switcher is determined according to input and output capacity, manufacturers' literature should be sought to determine the architecture of different brands of switchers. The architecture is the basic building block or unit that the manufacturer selects as the primary matrix size. Typical basic switchers might have matrix sizes of 20 by 5,32 by 8,40 by 15 , or 100 by 10 . All of these can be expanded for greater capacity, but the basic architecture may well determine the overall system cost by the manner in which expansion is accomplished. There are a few systems that can be expanded to 1,000 inputs by 1,000 outputs. The aid of the manufacturer is very important in understanding the expansion techniques, space requirements, and cost.

Switchers of all types are characterized by common performance specifications. These include bandwidth, differential gain, differential phase, horizontal and vertical tilt, crosstalk, and output drive capability. These terms are defined in many manufacturers' video switching literature and data sheets and should be consulted for specific definitions. The aid of the switcher manufacturer should be sought while determining the appropriate switcher for the specific application. 


\section{VIDEO MONITORS}

Monitors operate in a manner opposite of image tube cameras in that a scanning beam creates a visual scene on the monitor face. Monitors are similar to the home television receiver but generally have a wider bandwidth and do not have tuning circuits.

\subsection{Size}

The video monitor screen is measured diagonally from corner to corner and is expressed in the nearest whole number of inches. The monitor glass envelope has rounded corners so that actual image measurements are somewhat less than the stated monitor size. The size of the monitor screen determines the comfortable viewing distance. Too close a viewing distance can cause eyestrain as the eye attempts to search the entire screen, while too great a distance will not provide the necessary resolution of detail.

In assessment applications, security personnel should be able to reliably detect intruders in a secured area and should be able to respond immediately to an intrusion. This usually results in a console of assessment monitors and other security-related devices. The 22.8-cm (9-in.) video monitor, at a viewing distance of $1 \mathrm{~m}$ (39.37 in.) or less, provides adequate image detail and quick search times. Mechanically, two of the $22.8-\mathrm{cm}(9$-in.) monitors can fit easily into a standard $47.5-\mathrm{cm}$ (19-in.) wide equipment rack.

Monitors much larger than the 22.8-cm (9-in.) size will require greater search times and produce widerspaced scanning lines in which small detail can be lost. A monitor of less than the 17.7-cm (7-in.) diagonal measurement normally does not produce the same resolution as the $22.8-\mathrm{cm}(9-\mathrm{in}$.), and detail can be lost because the successive beam scans overlap each other.

\subsection{Resolution}

The resolution of the monitor should be great enough to display all of the resolution capability of the video signal created in the camera. Better quality monitors will usually have a greater resolution capability. The vertical resolution is primarily determined by the number of horizontal scan lines as long as the beam 'ize is not excessive. Since this is established by a standard of 525 scan lines per picture height in the United States, the resolution will be limited to about 340 TV lines of vertical resolution. The standard video monitor uses a magneric deflection system for beam scanning. The nonlinearities in the deflection system reduce the vertical resolution much the same as in the image tube camera.

A good assessment monitor should resolve $800 \mathrm{TV}$ lines of horizontal resolution. Since the edges of the monitor are different distances from the deflection center than the center of the monitor tube, the beam becomes out of focus. This reduces the resolution at the edges and at the corners. A monitor that has 800 TV lines of horizontal resolution at the center may have only $600 \mathrm{TV}$ lines at the corners. This should be considered a normal situation. Some of the more costly monitors may provide some dynamic corrections to the beam deflection circuitry to compensate for the defocusing, but that is not usually required. The assessment camera should be pointed so that the area of interest is near the center of the picture for the best resolution.

\subsection{Bandwidth}

The horizontal resolution is primarily limited by the video circuitry bandwidth. Based on the criterion of $80 \mathrm{TV}$ lines being equivalent to a $1 \mathrm{MHz}$ bandwidth, the monitor should have about a $10 \mathrm{MHz}$ bandwidth to produce the $800 \mathrm{TV}$ lines of horizontal resolution required. Monitor resolution considerably above the system bandwidth may introduce unwanted noise in the video scene, which can compromise rapid and accurate assessment.

\subsection{Gray Scale Response}

The video monitor should be capable of reproducing intermediate shades of gray between maximum and minimum video input signals. The standard Electronic Industries Association (EIA) resolution chart gray scale is capable of producing reflectances over a range of approximately 30 to 1 in ten steps of equal 
reflection densities. All monitors will reproduce a ten-step gray scale at some highlight intensity level. The highlight level is the brightest portion of the video image, comprising the background of the central circle in the EIA resolution chart.

\subsection{Dynamic Range}

The dynamic range of the monitor is defined as the range of highlight luminance levels that can be achieved while maintaining the complete 10 -step gray scale response. This dynamic range is important in determining the ambient light levels in which the monitor can operate. A monitor that can produce the full gray scale at only 10 to 20 footlamberts highlight luminance (not illumination) will be useful in significantly reduced room light only. If other activities are occurring in this environment, the room lighting may be inadequate. Some monitors will reproduce the 10-step gray scale at highlight luminances in the range of 40 to 50 footlamberts, which is adequate for fuily lighted offices and laboratories, but will be unacceptably brigisi in a dimly lit ros, m. A good quality monitor, having a range of highlight luminance from 10 to 60 footlamberts, should be selected when the ambient light level is unknown or should be varied. Higher luminance levels should be specified if daylight will contribute to the ambient light level. Maximum highlight luminance levels are seldom specified in manufacturers' data; none specify dynamic range.

\subsection{Voltage Regulation}

The power supply regulation in the monitor will affect the raster size and beam focus. Poorly or unregulated power supplies can cause the raster size to change and/or to become out of focus as the brightness and contrast controls are varied. The brightness control, a DC level adjustment in the beam conduction circuitry, should affect the overall scene luminance evenly across the picture. The contrast, a video signal gain adjustment, should change the range of brightness between the brightest and the darkest parts of the image. The better quality monitors will produce little or no effect on the raster size or beam focus as the contrast and brightness controls are varied throughout their range. This is a quick method of approximating the quality of the monitor, even without an input signal on the monitor. The useful dynamic range will depend upon maintaining good resolution at various contrast and brightness levels; however, it should not be the only criterion for monitor evaluation or selection.

\subsection{Automatic Gain Control}

An automatic gain control (AGC) feature is available on some monitors. This allows the monitor to correct for varying levels of input signal and maintain a stable monitor presentation. This feature may be useful in small assessment systems where video signal conditioning is not used. The use of AGC type monitors should not be a substitute for proper camera signal output level adjustment. The effects of the AGC should be investigated before final monitor selection.

\subsection{Underscan}

The more familiar video receivers or monitors have images that fill the entire screen. The boundaries of the image are beyond the edges of the screen, 80 it is not possible to determine the real size of the image as seen by the camera. For this reason, some monitors provide an underscan option by switch or adjustments that decrease the image size so that the edges are visible. Thus, the entire image as seen by the camera is visible.

Underscanning also maintains the image within the sharper focus regions of the monitor. Monitor adjustment by vertical and horizontal size controls can provide continuously variable amounts of underscanning not provided by the underscan switch selection. The use of underscanning; except for the exact determination of the image boundaries, provides no significant advantage in assessment. It appears to be a matter of personal preference. A properly adjusted monitor would not allow a significant

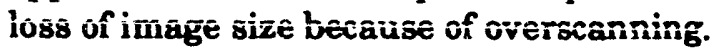




\subsection{Monitor Location}

The monitor should be located to provide easy visual access and to be within reach of any other securityrelated equipment. Frequently, monitors are tilted upward to be perpendicular to the viewer's line of sight. This may produce glare on the monitor face from ceiling lights located behind the viewer. In this case, the lights should be moved, turned off or shielded from the monitor face. Light from other directions can be similarly handled, or light-control film can be used to eliminate particularly troublesome sources of glare.

\subsection{Selection}

Monitors are typically selected by specification review, although many attributes mentioned above will not appear in specifications. A general guide to quality is the cost of the monitor. The CCTV industry is quite competitive, and there are many monitor manufacturers. Most monitors are grouped in the lower cost category with performance to match. The cheapest monitors should be avoided because of limited performance.

Some basic requirements are considered essential for the selection of any video monitor. The front and/ or rear panels should have the following controls:

- power switch

- brightness

- contrast

- focus

- vertical hold

- height

- vertical linearity

The monitor should have a video loop-through capability with input and output video connectors and a method for terminating the video line in a 75-ohm impedance, preferably by switch selection. An external sync input capability should be provided if a master sync system is to be used. This capability is quite common and typically includes sync loop-through connectors.

Optional controls could include horizontal linearity and underscan selection by switch, horizontal size adjustments, or in some cases a wiring change option in the deflection system to effect horizontal amplitude changes. Monitors are available that can operate from 115-volt and 230-volt AC line sources as well as from 12 -volt DC sources.

\subsection{Evaluation}

Several monitors meeting the system requirements should be acquired for evaluation. A more expensive monitor should be evaluated to determine the benefits of such a monitor for the specific apolication. A standard EIA reso ution chart and a high resolution camera can be used to drive a group of monitors simultaneously. A side-by-side evaluation will more readily indicate the better quality monitor than can recorded evaluation data taken at different times.

If possible, a multiburst signal generator should be used to determine the system resolution. Monitors specifying the same video bandwidth may not illuminate the monitor screens equally when driven by the same high-frequency burst signal. This is one area where the specifications alone fail to identify a superior or inferior product.

A photometer to measure the useful range of highlight luminance levels is also recommended for test equipment for monitor evaluation. 


\section{VIDEO RECORDERS}

A video recording system produces a record of significant events for immediate assessment or for subsequent retrieval. The record may be permanent or may only be temporary until an assessment of an alarmed area has taken place. If the use of the recording device is to capture the action of an intruder, the device should start recording well before the adversary completes crossing the assessment zone. Video-image capture devices store several images in a continuously updated sequence and automatically recall the images when an alarm occurs. The selection of a particular storage media is contingent on specific purpose and budget.

\subsection{Tape Recording}

The most common method of storing security image information is on magnetic tape. The electrical signal created in the camera, which represents the video image, is recorded on magnetic tape in much the same manner as in a voice recorder. For video recording, high writing speeds are required because of the wide bandwidth of the video signal. The video recording heads are mounted in a drum assembly and are rotated at high speed. The tape is wrapped around the drum assembly in a slightly skewed fashion with respect to the rotating recording heads and travels in a direction opposite to the rotation of the recording heads. The result is a recording track several inches long, running diagonally across the narrow tape, created by one recording head in the time duration of one video field. The next recording track is spaced very close to the first track, with only a narrow gap to provide signal isolation between adjacent tracks.

The magnetic tape recorder can store real-time video for several hours on a reel or cartridge, depending on the type of recorder. The more common reel-to-reel recorders use an Electronic Industries Association (EIA) J-1 format that has been available from many manufacturers. These EIA J-1 tapes are interchangeable between recorders. The reel-to-reel recorder has dropped in popularity because of the greater recording capacity and the tape handling ease of newer recording formats. The reel-to-reel recorder still has a few advantages, such as ease in searching for specific frames of data for image analysis and quick start-up from a standby record position. This allows the recorders to start recording more quickly after a command to record is given. The recording starts quickly, but the tape transport mechanism needs to come up to speed before stable recording is produced. It may take 2 to 3 seconds for the tape to reach the proper recording speed in reel-to-reel recorders.

Cartridge (cassette) recorders are available in three common recording standard formats. In the 1.27$\mathrm{cm}(1 / 2-$ in. $)$ tape width, the Beta and VHS formats are most common, although the Beta format is losing popularity in the consumer sector. While the consumer marketplace does not produce many products directly applicable to security use, it does provide the basis for many security-related developments in recording devices. Many types of recorders are available in the Beta and VHS formats for security applications. While tapes of any of the two formats are compatible from machine to machine within a given format, the Beta format tapes are not interchangeable with the VHS format tapes. In some specialized recorders, such as time-lapse recorders, the tape cassettes of a given format can be used on different manufacturers' recorders, but the tapes cannot be recorded and played back except on the same manufacturers' brand and model of recorder.

Currently the maximum recording time in the Beta format is $\mathbf{5}$ hours and in the VHS format 8 hours. Despite strong personal preferences for one format over the other, there is little significant difference in the resolution capabilities of the two $1 \bar{z} 7-\mathrm{cm}(1 / 2-i n$.) format recorders.

In recorders typically used in security applications, the Beta format is limited to 3 hours of recording time, and the VHS format recorders are limited to 6 hours of recording time. These recorders typically have remote control function of the major front panel controls. The type of recorder selected has more to do with the recording or playback features desired than the resolution capability differences.

The third type of cassette recorder is the U-matic format. It has a $1.91-\mathrm{cm}(3 / 4-\mathrm{in}$.) tape width and is used extensively in the commercial broadcast industry. This format has the advantage of a modestly increased resolution over the Beta and VHS format recorders. These recorders are capable of resolving about 300 TV lines of horizontal resolution, while the U-matic recorders can resolve about 350 TV lines. 
Both the recorders and the tape cassettes of the $U$-matic format are considerably larger in physical size. The maximum recording time on a single $U$-matic cassette is 1 hour, while the VHS format recorders can record up to 8 hours on a single cassette. There is little available in the way of modification of the U-matic cassette recorder for security applications, so it is not used as extensively as the VHS format recorders.

It should be noted that considerable time will be required to stop, rewind, and play back the recorded scene for assessment purposes. If repeat playbacks are required, the assessment time will be extended considerably. While one assessment is in progress, another sensor may be triggered that will also require assessment. The design of the intrusion detection system should include the consideration of circumstances involving many simultaneous alarms, which will all require assessment in a timely manner.

Some resolution performance improvements to the basic Beta, VHS, and U-matic formats are available or have been announced. Their effect on security applications has been minimal to date. Any increase in the resolution capability, however, would improve the overall assessment system performance, since the video tape recorder exhibits the lowest resolution of any of the video components.

A newer format developed primarily for electronic news gathering (ENG) is the $8 \mathrm{~mm}$ format. The $8 \mathrm{~mm}$ format uses a metal tape that results in improved resolution and recording density. The tape transport mechanism and cassette size are about one-third the size of the VHS format and are limited to 2 hours of recording. These advantages for ENG applications are obvious, but the reduced space and weight and increased audio fidelity are not an overriding concern in most security applications. Many other formats have been developed for ENG purposes but have had no impact yet on enhanced performance in video assessment or surveillance recording applications.

Standard tape recorders are not acceptable for making rapid alarm assessments from the recorded data because of the long time required for tape recorders to start recording after the record command is given. Reel-to-reel recorders may require 2 to 3 seconds to produce acceptable recordings while cassette recorders may take as long as 5 or 6 seconds. Rewinding and playback start times extend this delay to unreasonably long periods. Other types of recording devices can reduce the assessment time considerably.

\subsection{Disc Recording}

Video disc recorders are available in 2 wide variety of formats. The recording medium is similar to that of tape recorders except that the recording medium is on a single flat disc. Some disc recorders use a flexible Mylar ${ }^{\infty}$ disc while others use a rigid aluminum disc. The rigid disc is not readily exchanged between recorders but the flexible discs can be exchanged between recorders of the same manufacture. The recording heads may or may not touch the recording medium surface, depending on the technique used. A single revolution of the disc may record one field or an entire frame of video data.

In all configurations, the basic operating principles are the same. The magnetically coated disc is rotated at either 1,800 revolutions per minute $(\mathrm{rpm})$ for full-frame recording or 3,600 rpm for single-field recording. The head system may include discrete heads for erase, record, or playback, or a single multifunction head. The head records a single field or frame from a fixed position and then is stepped to the next location for a subsequent field or frame. In this manner, a stop-action playback can be achieved by indexing the head to the desired fixed location and allowing continuous playback of that particular recorded revolution of the disc.

In continuous-recording mode, the time interval required for the head system to move from one disc position (track) to the next may exceed the vertical blanking interval between fields of the video signal. A disc system of this type is not capable of continuously recording every field or frame. After recording a complete revolution of the disc and stepping to the next track, it waits until the next vertical blanking interval before continuing with the recording. This produces a jumping phenomenon during playback, but it is usually not objectionable to watch for short periods of time. 
Disc recorders use a frequency-modulated carrier recording system similar to that of the helical-scan tape recorders. Disc recorders have a bandwidth capability extending out to as much as $10 \mathrm{MHz}$ as compared to the $3 \mathrm{MHz}$ capability of the typical tape recorder.

Another type of disc recorder uses a continuous spiral recording track for real-time recording at 30 frames per second. It can begin recording in 100 to 300 milliseconds after an electronic command. The delay is determined by the time required to position the recording head onto or adjacent to the disc. The total recording time of this disc is limited to about 6 to 15 seconds. When used for video assessment, the image is retained until the alarm scene has been assessed and the disc recorder is ready for the next event. This type of recorder is used for the instant replay so popular in the sports broadcast industry. An added feature for this type of recorder is variable playback disc speed that produces the slow motion playback.

While considerably more expensive than the helical-scan tape recorder, the disc recorder can begin recording much more quickly than the typical cassette tape recorder. In the tape recorder, stable recording starts only after the tape is extracted from the cassette, has wrapped around the record drum, and reached full recording speed. This can take from 3 to 6 seconds, not quick enough for recording the penetration of a secured area by an intruder. The recorder can be left in a pause mode, with the heads at full speed and the tape stationary, but only for a few seconds without possible damage to the tape or recording heads or both. Reel-to-reel tape recorders can tolerate longer pause mode operation if the tape transport mechanism reliably kicks back the take-up reel to allow the tape to slacken away from the recording drum and the continuously rotating recording heads. This keeps the record heads away from continuous contact with the video tape, unlike the cassette recorder.

As previously stated, multiple alarms will require multiple recording devices, increasing the costs of the assessment system. Multiple disc recorders can be very expensive to purchase and maintain. Video disc recorders require considerable maintenance time and talent, which should be considered when selecting the recording device.

\subsection{Optical Disc Recording}

In place of the magnetic medium for recording, the optical disc recorder uses a laser beam to burn a signal into the storage medium. The data, thus stored, cannot be overwritten, nor are they subject to damage or destruction in a magnetic environment. The optical disc, however, cannot be reused as can magnetically recorded media. The data storage density is significantly greater in optical storage devices than on magnetic media. Some very large systems can store over three million video scenes. Because of their high cost, optical disc recorders have not yet been used in assessment applications. New developments in erasable/reusable optical disc technology are expected.

\subsection{Solid-State Recording}

Using computer-created technology, video images can be stored in solid-state memory. The capacity of a solid-state video memory is measured by the number of discrete points used in creating an image. The continuous horizontal scan line can be broken up into many discrete points, or picture elements. The number of picture elements (pixels) determines the resolution capability. The vertical TV image is already broken up into 485 separate scan lines creating 485 pixels in a vertical column. Each pixel has a unique position called an address in the video raster. The brightness (shade of gray) of each pixel can be represented by a discrete number. Thus we can convert an analog scene into a computer-based format by digitizing the video signal.

Early developments created video storage for slow-scan video transmission over standard telephone lines. The small memory capacity limited the resolution of the systems. Larger memory chips have increased resolution to the point that usable images can be stored for assessment purposes. 
An image storage capacity of 256 by 256 yields a resolution of about 175 TV lines vertically and about $200 \mathrm{TV}$ lines horizontally. This is adequate for assessment image storage as long as the minimum resolution requirements are considered when the assessment system is designed. This memory capacity seriously limits the resolution capability of the assessment camera and is less than the resolution capability of video tape recorders. A larger memory size (e.g., 512 by 512) will double the resolution capability to about $350 \mathrm{TV}$ lines of vertical resolution, the same as the camera, and yield nearly $400 \mathrm{TV}$ lines of horizontal resolution, somewhat greater than the better tape recorders.

Although large digital memories exist, the amount of data in continuous, real-time video recording could rapidly fill a mass memory. Instead of continuously recording an event, a few single fields of video, recorded at much slower rates, are acceptable for alarm assessment. This concept is sometimes referred to as 'video-image capture.' For example, if one field of video is stored when an alarm is triggered, and a second field is stored after several field times have elapsed, the image can be combined in a real-time playback mode. If an intrusion has taken place, the combined image will show the intruder at both times that the field images were recorded, in an exaggerated-motion, continuous playback. In a similar fashion, one complete frame can be compared to another, but requires a doubling of the memory size for the dual image. It should be noted that in the two-field method the intruder resolution is half that of the full-frame method although the image size is the same. When the two fields are combined, the total stationary scene has the full resolution capability. Video-image capture systems using a four field format are also available and can greatly enhance alarm assessment capabilities.

Another useful recording mode available in some solid-state video recorders is continuous interval recording. After the memory is filled, the oldest previous image is overwritten and lost. When an alarm message is received, the interval recording stops at a programmed time such that the retained image sequence for automatic playback contains video images immediately before ("pre-alarm recording") and after the time of the alarm. This mode is particularly suited for video systems with relatively slow switching speeds. The number of images in the sequence and the time interval between recorded images can be selected by the user. The memory can also be partitioned to record multiple simultaneous alarms for sequential, prioritized playback and assessment.

Playback/display modes include sequential images on a single monitor, a quad display on a single monitor, and single images on multiple monitors. The sequential playback on a single monitor tends to attract attention to the portion of the scene where change (intruder motion) is present.

The cost for a solid-state recording system depends on the number of fields or frames retained until assessment is completed. This is determined by the system designer's perception of the number of simultaneous alarms that the system will handle. Small sites require fewer solid-state memories than larger sites, since the number of sensors will be smaller, leading to assumed lower false and nuisance alarm rates.

Mass memories have a large capacity for image storage at a lower cost per memory than discrete memory units. The number of simultaneous inputs and outputs restricts their capability to handle multiple alarm inputs or to provide simultaneous outputs to assessment monitors. A balanced number of smaller mass memories may be an acceptable compromise between cost and simultaneous input/output capabilities.

\subsection{Archive Recording}

The primary function of archive recording is the long-term storage of alarm assessment and special event video information. The video data can originate directly from an assessment or surveillance video camera or from other recorded media. The typical archive data will be in relatively short segments of real-time recordings. The recorder can be any type of ordinary video recorder except that it should be under automatic control when recording to assure the collection of all the video information available. 
Since archive recording is primarily intended for relatively long-term storage of large amounts of video information, the videocassette tape recorder is the preferred choice for most applications by reason of equipment cost and storage volume. Archive recording can provide an accurate chronology of events in multiple alarm situations when assessment functions may significantly lag behind the alarm reporting system. Post-event analysis can provide a significant amount of information on the effectiveness of the entire intrusion detection system. Taking full advantage of these capabilities will require a careful analysis of the design goals and the implementation necessary to achieve these goals.

Archive recording can also indicate the date, time, and location of alarms to separate nuisance alarms from false alarms. Over time, these data can provide a reasonable indication of the effectiveness of an individual sensor as well as the entire intrusion detection system under varying environmental conditions.

Time-lapse recorders are tape recorders that record one field per head revolution as in the standard video tape recorder, but the time between successive recorded fields is extended. The delay between recorded fields can be varied to allow extended recording time from a single cassette.

Time-lapse recorders were first available in reel-to-reel recorders with a maximum recording time of about 108 hours on a standard 1-hour tape reel. Currently, the most widely used time-lapse recorder is the VHS tape format using the popular 2-hour cassette. Recording times have been extended to 480 hours with VHS compatibility and 999 hours on special formats from a 2-hour (T-120) VHS cassette.

Time-lapse recorders are not used for video alarm assessment, but more commonly for long-term surveillance. Time-lapse recording can be a valuable tool in determining and documenting an intrusion into a protected area and any activity that has taken place. Tape playback is normally made long after any event.

Most time-lapse recorders have the capability to switch from a time-lapse mode into a faster near realtime mode when activated by an external circuit, such as a sensor contact. The intent is to provide a more detailed record of a specific event. Many such specific events will significantly reduce the amount of total recording time on a single tape cassette. When using this mode of operation, the remaining tape supply should be monitored frequently to avoid running out of tape unknowingly. To avoid this problem, some time-lapse recorders have a feature to start a second recorder when the first recorder runs out of tape or to provide an end-of-tape warning.

Selection of a maximum recording time should include a calculation of the time between recorded fields of video data. If the time between fields is too long to obtain the necessary detail of activity, then the maximum recording time should be reduced. For example, in real time a field of video data is produced 60 times each second. A selected maximum recording time of 120 hours using a 2-hour cassette will yield an image once each second. If a suspected event can take place in 1 second or less or a more detailed analysis is required, then a shorter maximum recording time should be selected.

Time-lapse recorders are available from several manufacturers and should be selected on the basis of the operating characteristics and functions required for the specific application. Some time-lapse recorders can be computer controlled through an RS-232 port on the recorder or by a separate controller box. 


\section{INFORMATION DISPLAY EQUIPMENT}

Time/date generators, source identifiers, and environmental data generators are all examples of data insertion generator equipment that convert electronic signal information into an alphanumeric video waveform for presentation on closed-circuit televisiou (CCTV) monitors and video recorders.

While the equipment differs by function, some operating characteristics are common among these devices. All require the video signal to pass through the device for insertion of the data into the video signal. Some of these devices have active electronic components in series with the video input. In the event of failure of the data insertion generator, the video signal path is interrupted, and no video output signal is available at the output jack of the data insertion generator. These devices should be avoided unless other operational characteristics mandate the selection of this type of data generator.

A data-insertion generator that adds the insertion information passively, as by resistor network, does not interrupt the input video signal in the event of insertion generator failure. This type of device is recommended if all other requirements for the generator are met. Some of the data insertion generators may require external sync to operate. This can be a serious restriction if a master sync signal is not required in the rest of the video system. Careful selection of the data insertion equipment can avoid this requirement.

Many of the data insertion generators have data inserted at any brightness level selected. This will allow the inserted characters to be varied in brightness between black and white for the necessary contrast with the background video information. Some of the devices have an edge enhancement around each letter, usually black, which provides the best readability against any type of background. This characteristic is recommended when selecting the device. With fixed position assessment cameras, it is usually possible to find some area in the field of view that will contrast with the inserted data even if the edge enhancement feature is not available.

\subsection{Time/Date Generators}

Time/date generators display the time and date on a video monitor or insert the information in the video signal transmitted to a video recorder. Time/date generators are particularly important for archive recording, where the fact of an event may be of little value without identifying the time of the event. The time is usually displayed in hours (12- or 24-hour format), minutes, and seconds. Some generators may also provide time to tenths and even hundredths of a second. The date is usually numerically displayed by month, day, and year. These generators have the ability to keep track of the different lengths of the months and to correct for the additional day in leap year.

The central timing source for the time/date generator is either the $60-\mathrm{Hz}$ line frequency or an internal crystal-controlled oscillator. The power line frequency from large commercial power systems is quite accurate when averaged over long periods of time. The basic timing circuitry can be provided with a battery backup option to prevent loss of time information during power interruptions. The battery may be either a replaceable alkaline or lithium type or a rechargeable nickel-cadmium type. The nickelcadmium type requires a continuous floating charge system to maintain full battery capacity while the power is supplied from the alternating current (AC) line. The battery should be able to maintain the timing circuitry from several days to a few months, depending on the system. The battery can maintain the timing circuitry only and will not be able to provide the power for the character insertion circuitry.

\subsection{Source Identifiers}

Source identifiers are data-insertion generators that provide annotation to the video signal to identify the camera or the sensor sector viewed by the camera. The identification can be by either arbitrary numbers or word description.

Some source identifiers are individual units with alphanumeric programmable character generators that resistively add the programmed annotation to the video signal. These are normally placed in the video signal after the signal conditioning equipment (if used) but before any distribution or switching 
equipment. This has the advantage of identifying a video source at any point in the distribution, switching, display, and recording network.

Another advantage of the individual unit is that one manufacturer offers a video presence detector and source identifier combination unit. The source identifier is limited to a four-digit, user-selectable number, and the presence detector is a full-function high and low video average picture level(APL) detector.

Other types of source identifiers are computer-controlled and create the annotation at the time that the video input is selected. These types can provide a greater amount of annotated information than the individually programmed units. The computer-controlled source identifiers should be placed at the output of the video switcher to provide annotation to video monitors or recorders. With this type of annotation, the video lines are not identifiable except between the source identifier and the monitor or recorder.

Though more expensive than the individual identifiers, fewer of the computer-controlled source identifiers are usually required. A video assessment system with relatively few inputs (cameras) and a large number of outputs (monitors and recorders) can effectively, with respect to cost, use the individual source identifiers. As the number of inputs increases significantly, or the number of outputs decreases, the cost of the computer-controlled source identifiers becomes less. The cost of the programming required for the computer-controlled identifier should be considered in the cost analysis.

\subsection{Weather Data Indicators}

Environmental data generators provide alphanumeric display of various environmental conditions such as wind speed and direction. Output data from the weather sensors are digitized and processed through an alphanumeric character generator for subsequent insertion into the video signal. Weather data, when supplied to an archive recorder, can provide a considerable amount of information on the operation of individual sensors as well as the effectiveness of the entire intrusion detection system under a variety of identifiable, documented environmental conditions. 


\section{MISCELLANEOUS ELECTRONIC EQUIPMENT}

Numerous other components are typically necessary to complete a video assessment system, including power and signal line surge suppressors, protective devices for cables and equipment, and emergency power supplies.

\subsection{Surge Suppressors}

Lightning and sudden changes in ground potential can readily damage input circuitry in wire-cabled video transmission equipment. Systems using multiple cable distribution, such as camera sync distribution networks, are especially vulnerable to significant damage because of the large amount of equipment connected to these lines. In any area with more than a few lightning days per year, some form of lightning protection is normally essential to protect the system components from lightning strikes at or near a system component.

Surge suppressors can effectively clamp the maximum voltage appearing on a conductor to safe levels. Several types of commercial surge protection devices are available; the most common form utilizes Zener diodes and current-limiting resistors connected across the conductors or from the conductors to ground.

Any type of diode used for surge suppression will have some inherent capacitance. The diodes should have as small a capacitance as possible to reduce the high-frequency roll-off of the video signal resulting in a loss of resolution. Two or more diodes in series will reduce the effective shunt capacitance from the video line to ground. The clamping voltage for surge suppression should be closer to the actual operating voltage on signal lines than is necessary on power lines. The effect of a surge suppression device should be evaluated or demonstrated on an active video line before the final selection is made.

External line power may also require lightning and/or surge protection at the video equipment location. Protection is normally in the form of gas discharge tubes (spark gaps), which limit the line voltage to a safe level if the line voltage suddenly rises above normal operating range.

Typically, a combination of Zener diodes and gas tubes is used because Zener diodes are fast response, low current devices that protect a component from the fast current spike of a lightning strike. To handle the large currents from lightning, tubes are used to dissipate the bulk of the current.

\subsection{Video Presence Detectors}

Video presence detection is to video transmission lines what line supervision is to alarm communication lines: the continual monitoring of sensitive signal lines to detect unauthorized monitoring, tampering, or signal interruption. In large video assessment systems, not all of the camera outputs will be displayed often enough to notice a loss of signal. The video presence detector can provide this continuous monitoring function.

Video presence detectors can be designed to detect only the loss of the sync portion of the video signal. This type will indicate an alarm status if a total loss of video signal occurs. This type of presence detector is adequate for determining the intentional or accidental failure of the camera or video equipment and cables up to the presence detector. In some cases, the camera might be covered by snow or spray paint or blinded with a bright light. The simple sync presence detector will not indicate an alarm status under these circumstances, since the sync signal is still intact. The more sophisticated video presence detectors can detect changes (increase or decrease) in the average video signal level from preset levels. If the video signal increases or decreases in average picture level (APL), the presence detector will indicate an alarm, usually by closing or opening a set of relay contacts. The choice of the alarm type (normally open or normally closed) is left to the video system designer.

APL detection adjustment should be carefully established and performed after the camera is positioned for the best view of the assessment area. The set points of the detection levels should be made for day and night operation of the camera to minimize the number of false alarms while still providing certain operation with scene brightness changes. If the assessment area is properly illuminated, the auto-iris 
lens and camera automatic gain control (AGC) circuitry should compensate for scene brightness changes from day to night operation.

Video presence detectors are highly recommended for use on any video line that is not totally contained within a protected area. When the camera towers are located outside of the sensor line in a perimeter assessment system, video presence detectors can provide the line supervision function.

The video presence detector is normally located in a protected area, usually where the signal conditioning and video switching equipment is located. If inserted in the video line after the signal conditioning equipment, the presence detectors also monitor the operation of the signal conditioning equipment. This is the preferred location since all of the video levels should be adjusted for proper and similar amplitudes at this point. The presence detectors are easily adjusted if all of the video levels are of similar amplitude.

A single presence detector is often used for each incoming video line. In systems where a large number of cameras are used, the cost of individual presence detectors is prohibitive. In this situation, a portion of the video switcher can be used to sequence all the video input signals through a small number of video presence detectors. Each video input is switched to a presence detector for a relatively short time before being switched to successive video inputs. The time of the sequence for any individual presence detector should be short enough to allow timely reporting of a loss or significant change in the video level of any of the input signals. This technique will extend the alarm reporting time, but it reduces the number of presence detectors required and reduces the cost of presence detection. It does, however, require additional video switcher capacity, programmed sequential switching, and a more complicated presence detector alarm system. The cost of both techniques should be evaluated to determine the approach most consistent with the assessment system requirements. In either method, the use of video presence detectors should not degrade the quality of the video signal or cause loss of video in the event of presence detector failure.

\subsection{Tamper Protection}

Some ancillary equipment near the camera end of the video system may require protection from tampering. Camera junction boxes containing power distribution, signal conditioning equipment, lightning protection circuitry, and other equipment should be protected with tamper alarms to warn of intrusion into the camera circuitry. Typically, these camera junction boxes are located on or near the camera tower and should not be of such a size as to provide cover for an intrusion into the sensored area.

\subsection{Emergency Power Supplies}

Some or all of the equipment in a video assessment system should be able to operate when the main source of power is interrupted. The reliability of the main source of power and the requirement for continued, unrestricted operations will determine the amount of emergency power capacity needed. As a minimum, backup power should be provided for timing and video switcher circuitry; both are examples of equipment containing volatile memory storage information. Fortunately, many of these devices have battery backup as an optional feature and should be considered when selecting any type of equipment.

If emergency power is not provided for assessment system lighting, it may not be necessary to provide emergency camera power except for daytime operation. Specific equipment can be isolated for emergency operation in the event of primary power failure. The needs of the specific site and the funds available for emergency power will determine the extent of the protection provided. Without emergency power, alternate means of assessment should be provided during periods of power loss. 


\section{SYSTEM CONSIDERATIONS}

The design of an effective intrusion detection and assessment system should not be attempted without the assistance of a number of persons proficient in many technical areas. The interaction of these disciplines should be understood in order to create an effective intrusion detection system.

\subsection{Sensor Compatibility}

In the design phase of an intrusion detection system, the sensored areas and the assessment camera capabilities should be considered together to avoid camera placement that would interfere with or compromise sensor effectiveness. Camera tower placement should not be so close to the sensor detection area or volume as to create nuisance alarms, decrease sensor sensitivity, or provide an aid to bridging the detection zone.

At the same time, the assessment camera should be able to view the entire sensored area or volume, including any sensor hardware associated with that area. In multiple sensor configurations, the assessment camera should be able to view the combined sensored areas and volumes as well as all of the hardware associated with those sensors. Sensor hardware should not be large enough to provide a convenient hiding place for intruders in the assessment areas.

\subsection{Sector Design Efficiency}

Camera sensitivity should be considered when designing the lighting system. The highest resolution camera will not function properly with inadequate illumination. The lens relative aperture, i.e., fnumber, should be considered in these determinations. Generally, the faster lens speeds (larger aperture, smaller $f$-number) should be selected when more than one option is available, even if the faster lens is somewhat more expensive. Lenses with $f$-numbers greater than $f / 1.8$, even zoom lenses, should not be used except under very unusual circumstances. These larger $f$-numbered lenses will likely require increased lighting levels or a more sensitive, thus more expensive, camera. A trade-off is that lenses with a smaller $\mathrm{f}$-number have a smaller depth of field.

Camera sensitivity often cannot be judged from manufacturers' specifications. There appears to be little consistency in providing data referenced to a common standard. Some manufacturers reference scene illumination levels using incandescent light sources with the recommended and/or minimum usable light levels. These light levels are, by definition, subjective and have little value when comparing different cameras for performance. A side-by-side evaluation of cameras operating in the intended environment is the preferred way to determine each camera's suitability.

Video assessment system resolution determines the usable length of assessment sectors. Since the system resolution is a function of the resolution of the cameras, transmission system, surge and lightning suppression components, video switcher, monitors, and, in some cases, the alarm event recorders, each of these elements should be considered individually as well as together. If assessments are to be made from recorded data, the resolution of the recording device should be considered, which may require reduced spacing between cameras, reduced maximum sector lengths, because of lower resolution. Typically, the image storage device will be the resolution limiting factor in the assessment system. If significant data are to be retrieved from archive recording, then the archive recorder resolution should be considered in the system design.

The most efficient use of perimeter assessment cameras results when the longest standard focal length lenses are used. The longer focal lengths have narrower fields of view that reduce the area viewed outside the clear zone. This minimizes the number of unwanted light sources that can cause degraded camera performance. The longest standard fixed-focal length auto-iris lenses at this time are $75 \mathrm{~mm}$. In the system design process, the longer focal length lenses should be tried first to determine effective assessment area and distance. Only if the longer focal lengths do not fit the assessment area should shorter focal lengths be tried. Since the number of standard focal lengths is limited, the video assessment system designer should work with the sensor system designer to provide the most efficient use of resources. 
Sector width also has an effect on the maximum usable sector length, with narrower widths providing the longer sectors. Minimum sector width will usually be decided by the sensor limitations, but unusual site configurations may also limit the desirable sector width.

Assessment cameras can view more than one sensor sector in some circumstances. Sensor sectors shortened by terrain variations or personnel access points can effectively save an assessment camera and associated circuitry and hardware under these circumstances. Each circum itance should be judged individually to determine the least expensive approach that should not compromise effective and timely assessment.

\subsection{Equipment Location}

Most video assessment equipment can be located in a central interior facility for convenience and ease of access for maintenance. Normally, only the cameras should be located at the remote assessment area. It is appropriate from a technical standpoint to locate some signal conditioning equipment at the camera site: pre-equalization equipment could increase the transmission line signal-to-noise ratio of the incoming video signal over post-equalization equipment. Ease of adjustment of the equalization equipment is the primary reason for locating this equipment in the central facility. This area requires access for maintenance personnel but can exclude guard force personnel.

The video assessment monitors should be located in an area where they can be easily viewed by guard force personnel. The location of the assessment monitors should not be compromised when additional safety and security equipment is located in the same console.

\subsection{Maintenance}

Maintenance procedures and equipment should be considered when the initial design of the assessment system is begun. The long-term success or failure of the system will depend on effective maintenance. Selection from equally performing equipment can and should be based on the ease of maintenance. Purchase of operational spare parts should be made at the same time as the primary equipment purchases to assure system operational continuity and interchangeability of parts. The decision of the level of maintenance to be performed on-site should be made early in the design process. This will determine the type of spare parts to be purchased and the required contracts for external maintenance and repair functions. Field replacement of defective hardware followed by repair center troubleshooting is the usual practice to minimize downtime and the need for compensatory measures.

A staff of qualified maintenance personnel is essential for the assessment system to continue to perform to the standards established during the system design. Ideally, an experienced maintenance staff should contribute to the system design and be knowledgeable about the system installation and checkout procedures.

Diagnostic equipment should be designed into the central facility equipment racks. The diagnostic equipment should include a waveform monitor (special purpose oscilloscope for video applications), a test signal generator, and separate video monitors. Video switcher equipment should include a full-function manual switcher control panel and a dedicated output bus for video system maintenance. Patch panels for access to incoming and outgoing video signals can provide ease of maintenance and adjustment procedures. Patch panels at the inputs and outputs of the video equipment racks can provide a convenient interface point where fiber-optic transmission systems are used.

A maintenance log of all equipment repairs and adjustments should be kept to provide a historical record of the actions taken to correct a specific problem. Maintenance trends can be established to identify recurring problems. This can substantially reduce repair time and identify equipment performing in a substandard manner. 


\subsection{Documentation}

Adequate maintenance cannot be performed without documentation of the equipment in use. Maintenance and operations manuals should be purchased at the time of equipment procurement. Complete documentation should include theory of operation, functional block diagrams, as-built drawings, cabling diagrams, schematic diagrams, and parts lists with manufacturers' and commercial equivalent part numbers. The selection of equipment for procurement should be based on the availability of complete documentation. 


\section{GLOSSARY}

active lines means scanning lines in the raster that contain video information.

$A G C$ means gutomatic gain control. An AGC circuit is used to maintain an output signal at a constant level over a limited range of input signal levels.

angular field of view means the measure of the field of view of a lens or camera/lens combination expressed in degrees.

aperture means the lens opening that determines the amount of light that will pass through the lens and onto the image plane.

aspect ratio means the ratio of the width to the height of the image in a camera imager or on a video monitor face. Current television systems have an aspect ratio of 4 to 3.

auto-iris lens means a lens whose aperture is controlled by monitoring the video signal amplitude and automatically adjusting the iris to maintain a constant video amplitude output.

baseband means the band of unmodulated frequencies, generally $30 \mathrm{~Hz}-10 \mathrm{MHz}$, containing all video signal information that may be used to modulate a higher carrier frequency.

black level means that level of a video waveform that represents the peak black portion of the image, established to be 7.5 IRE units from the blanking level.

blanking means the process of cutting off the electron beam in an image tube camera or in a video monitor at the end of each scan line and each field.

blanking level means that level of a video waveform between the video signal and the sync signal, established to be 0 IRE units.

blooming means the loss of detail in regions of the picture where the brightness is at an excessive level, because of enlargement of spot size and halo effect on the fluorescent screen of the cathode-ray picture tube; also, charge migration on video camera imagers from high illumination sources.

bridging (or looping) input means a high impedance intermediate termination of a signal line at the input of an amplifier, monitor, or video switcher where the signal line must continue on to other equipment. In video equipment, all outputs have a characteristic impedance of $75 \mathrm{ohms}$. The bridging (or looping) high impedance input allows several pieces of equipment to use a common video signal without significant loss of signal amplitude. The last input in a single video line must be terminated in 75 ohms.

brightness (luminance) means the attribute of visual perception in which an area appears to reflect or emit light, measured in footlamberts.

broadband means wide bandwidth trar,smission system with a single carrier and multiple information channels; it can be fiber-optic or RF (radiated or on cable).

inarge coupled device (CCD) means one of several types of solid-state imaging chips having discrete lightsensitive elements.

clamper means a device that maintains a video signal at a fixed potential to eliminate low-frequency interference; used primarily for common mode noise (hum) rejection.

closed-circuit teievision (CCTV) means a television system in which the signal distribution is limited or restricted, usually by cable. 
C mount means a standard threaded lens mount configuration having a 1-inch diameter and 32 threads per inch.

composite video signal means the combined picture signal, including video, vertical and horizontal blanking, and synchronizing signals.

contrast means the ratio of light and dark portions of a TV picture.

depth of field means the maximum distance from a lens that two objects may be separated in a given lens field of view and still maintain acceptable image focus for both objects.

distribution amplifier means a wideband amplifier having a single input and several impedancematched outputs for driving multiple signal lines. Distribution amplifiers can be used for video or sync signals.

EIA means Electronic Industries Association. This association has prepared standards that are in common use throughout the television industry (previously RETMA).

equalization means the process of correcting for transmission line losses of the frequency characteristics of an electronic signal.

equalizer means an electronic device used to compensate for low-and high-frequency losses through a transmission system.

field means the video produced in one vertical scan of the camera imager or monitor that consists of 262.5 horizontal scan lines in U.S. television systems. Two fields are required to form a single video frame.

field frequency means the rate at which video fields are created, nominally 60 times each second in U.S. television systems.

field of view means the area visible through the lens of an optical instrument. (See also linear field of view ar.d angular field of view.)

$f$-number means the ratio of the focal length to the clear aperture in a lens, expressed in the form $f / 1.8$.

focal length (FL) means the distance from the optical center of a simple lens to the plane of focus and is indicative of the image size produced.

focus means the process of adjusting the optical and/or electrical adjustments in a camera or monitor to obtain a sharp, clear image on the monitor.

footcandle ( $f c$ ) means a unit of illuminance equal to 1 lumen incident upon 1 square foot.

footlambert $(f L)$ means a unit of brightness equal to the amount of light reflected from a perfectly diffusing surface having 1 footcandle of incident illumination.

format means the size of the usable image in a TV camera or lens, or the system used to record video.

frame means a single television picture, made up of two interlaced fields, occurring in $1 / 30$ of a second, and consisting of 525 horizontal scanning lines (U.S. standard).

frame frequency means the rate at which a complete frame is scanned, nominally 30 frames per second.

f-stop means the lens designation indicating relative diaphragm opening or aperture diameter.

gamma means the numerical value that indicates the relative response of an electro-optical system to various levels of illumination. 
genlock means the condition where a camera or sync generator time base is controlled from an external source.

gray scale means variations in 10 steps from white through shades of gray to black as an established portion of the RETMA chart, used in qualitative measurements of video camera and monitor parameters.

horizontal blanking means the pulse starting at the end of each horizontal line that cuts off the beam during horizontal retrace, occurring at the rate of $15,750 \mathrm{~Hz}$ (nominal).

horizontal retrace means the repositioning of the electron beam in an image tube camera or video monitor between each horizontal scan, occurring during the blanking interval.

hum means the imposition of the power line waveform on a video signal.

hum bars means wide horizontal stripes resulting from hum (power line) interference. The bars may be in a fixed position on the monitor or may move vertically (roll).

illuminance see illumination.

illumination means the luminous flux per unit area incident upon a surface expresses in footcandles or lux.

image tube means an electron-beam tube used in a television camera where an electron current or a charge-density image is formed from an optical image and scanned in a predetermined sequence to provide an electrical signal; also called pickup tube.

interlaced scanning (interlace) means a process of interweaving two separate fields of video information to form a single video frame.

IRE unit means a standard amplitude used in video measurements equivalent to 7.14 millivolts. Stanclard video amplitude is $140 \mathrm{IRE}$ units (1 volt) consisting of $100 \mathrm{IRE}$ units (0.714 volt) of video and 40 IRE units ( 0.286 volt) of sync.

iris means the adjustable opening that controls the amount of light exiting a lens. Its diameter controls both the amount of light used to excite the imager and the depth of field.

light level means the intensity of incident light measured in footcandles or lux.

linear field of view means the measure of the field of view of a lens or camera/lens combination expressing the width or height of the scene at a specified distance, stated in meters (or feet).

line-lock means a condition occurring when the vertical drive (sync) is the same frequency as the power line.

lumen $(\mathrm{lm})$ means the photometric unit of radiant power. One lumen is the amount of luminous flux emitted into a solid angle of 1 steradian by a point source whose luminous intensity is 1 candela.

luminance see brightness.

$\operatorname{lux}(l x)$ means the metric unit of illuminance equal to 1 lumen incident upon 1 square meter. One lux is equal to 0.0929 footcandle.

magnification means the apparent enlargement of an object by an optical instrument, expressed as the ratio of the image size to the object size.

monochrome signal means a television signal without color information. 
multiburst means a waveform that contains a reference white signal followed by a number of discrete frequency signals in the period of one horizontal line.

Newvicon means the registered trademark for a low light level, monolithic-surface image tube, comparable in sensitivity to the silicon array image tube.

NTSC means National Television Systems Committee, a technical group that worked with the Federal Communications Commission in establishing television standards in the United States.

pan / tilt mount means an electromechanical device used for remote camera positioning.

peak-to-peak means the overall video signal amplitude between positive peak (white) and negative peak (sync tip) in a video waveform.

pixel (picture element) means the smallest resolvable detail that can be detected in a TV system, equivalent to one TV line of resolution.

$P T Z$ means a pan-tilt-zoom equipped camera.

raster means the pattern scanned by the electron beam; generally refers to the illuminated area produced by the scanning lines on a television monitor when no signal is present.

reference black level means the video waveform level representing the maximum limit for black peaks, established to be 7.5 IRE units above the blanking level.

reference white level means the video waveform level representing the maximum limit for white peaks, established to be 100 IRE units above the blanking level.

resolution means the degree to which closely adjacent parts of an image can be differentiated.

resolution (horizontal) means the number of independently resolvable elements in three-fourths of the picture width. It is most easily and frequently measured with the aid of a resolution chart, with the resolution units specified in TV lines. Horizontal resolution depends upon the high-frequency phase and amplitude response of the camera, transmission system bandwidth, and the monitor, as well as the size of the scanning beams in the camera image tube and monitor.

resolution (vertical) means the number of independently resolvable elements in the picture height. It is most easily and frequently measured with the aid of a resolution chart with the resolution expressed in TV lines. Vertical resolution is primarily fixed by the number of horizontal scanning lines per frame, generally considered to be about 350 TV lines in a 525 scan line system.

retrace means in an image tube or video monitor, the return of the scanning beam to its starting point.

roll means the vertical movement of an image on a monitor as the result of a temporary loss of vertical sync, frequently present in nonsynchronous switching systems.

scanning means the process of moving the electron beam of an image tube or CRT across the target or screen area of the tube.

signal conditioning means compensation for signal degradations caused by high-frequency attenuation, induced noise, and phase shifts in a video transmission path.

spatial frequency means a measure of resolution based on the number of alternate black and white lines in a specified distance; can be expressed as line pairs per millimeter as in optical measurements or in TV lines as in television systems. 
synchronization means the process of maintaining two or more scanning systems in phase.

sync level means that level of the video waveform that represents the maximum value of the sync pulse, established to be 40 IRE units below the blanking level.

sync pulse means a pulse transmitted as part of the composite video signal to control scanning.

underscan means a video monitor adjustment that decreases image size so that the edges are visible.

vertical blanking means the cutting off of the electron beam in an image tube camera or in a video monitor during vertical retrace, occurring at the rate of $60 \mathrm{~Hz}$ (nominal).

vertical retrace means the repositioning of the electron beam in an image tube camera or video monitor between each vertical scan, occurring during the blanking interval.

vidicon means a camera image tube in which a charge-density pattern is formed by a photoconductor that is scanned by an electron beam.

waveform monitor means an oscilloscope used specifically for measurement and analysis of video signals.

zoom lens means lens with a variable focal length. 


\section{DISTRIBUTION:}

Paul E. Ebel (2)

BE Inc.

P. O. Box 381

Hwy 278, Airport Industrial Park

Barnwell, SC 29812
6400
9540
9543
9543
9543
3141
3151
D. J. McCloskey
D. S. Miyoshi, Acting
J. C. Matter (2)
D. A. Greenwoll
D. C. Krukar
S. A. Landenberger (5)
G. C. Claycomb (3)
8523-2 Central Technical Files 


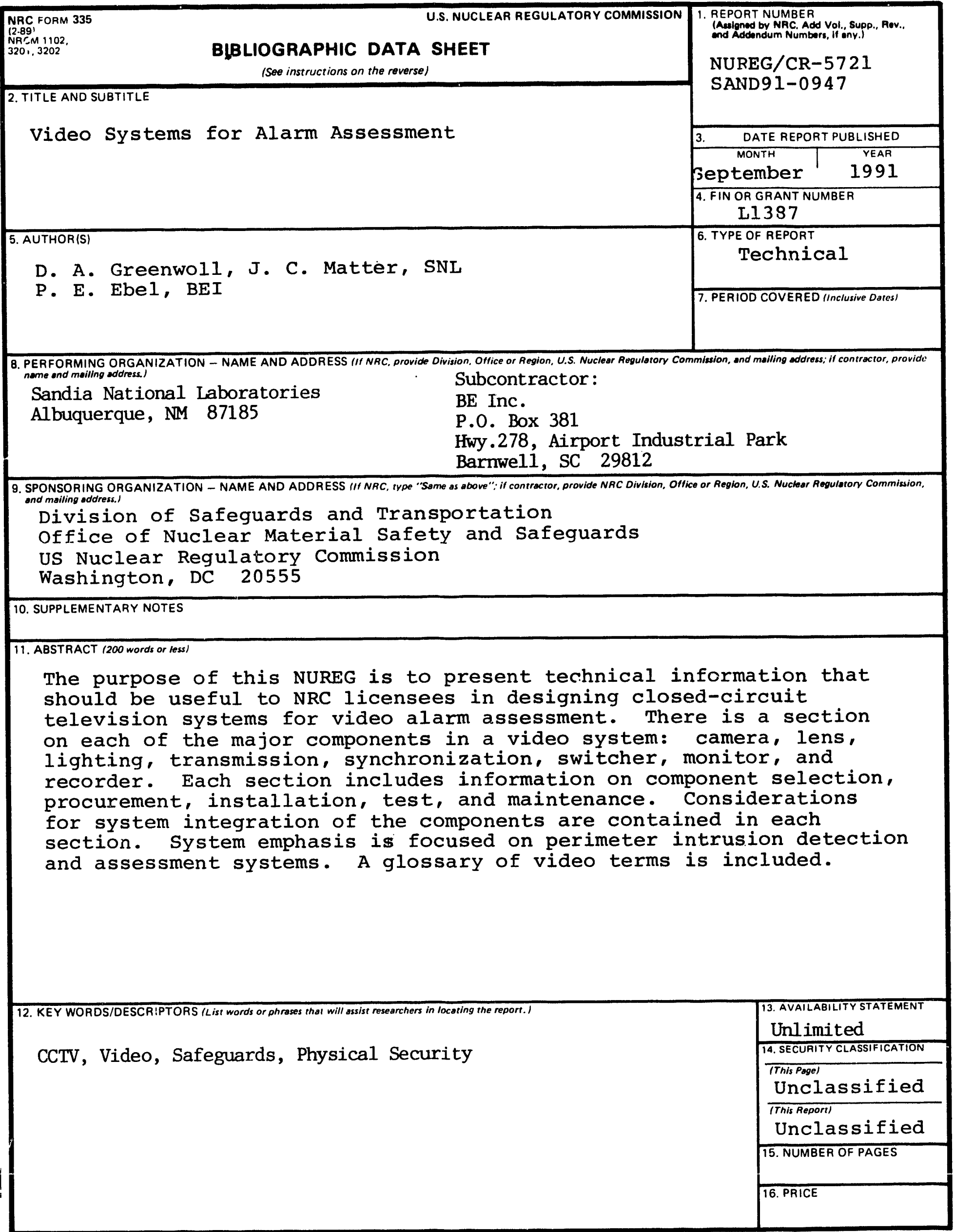



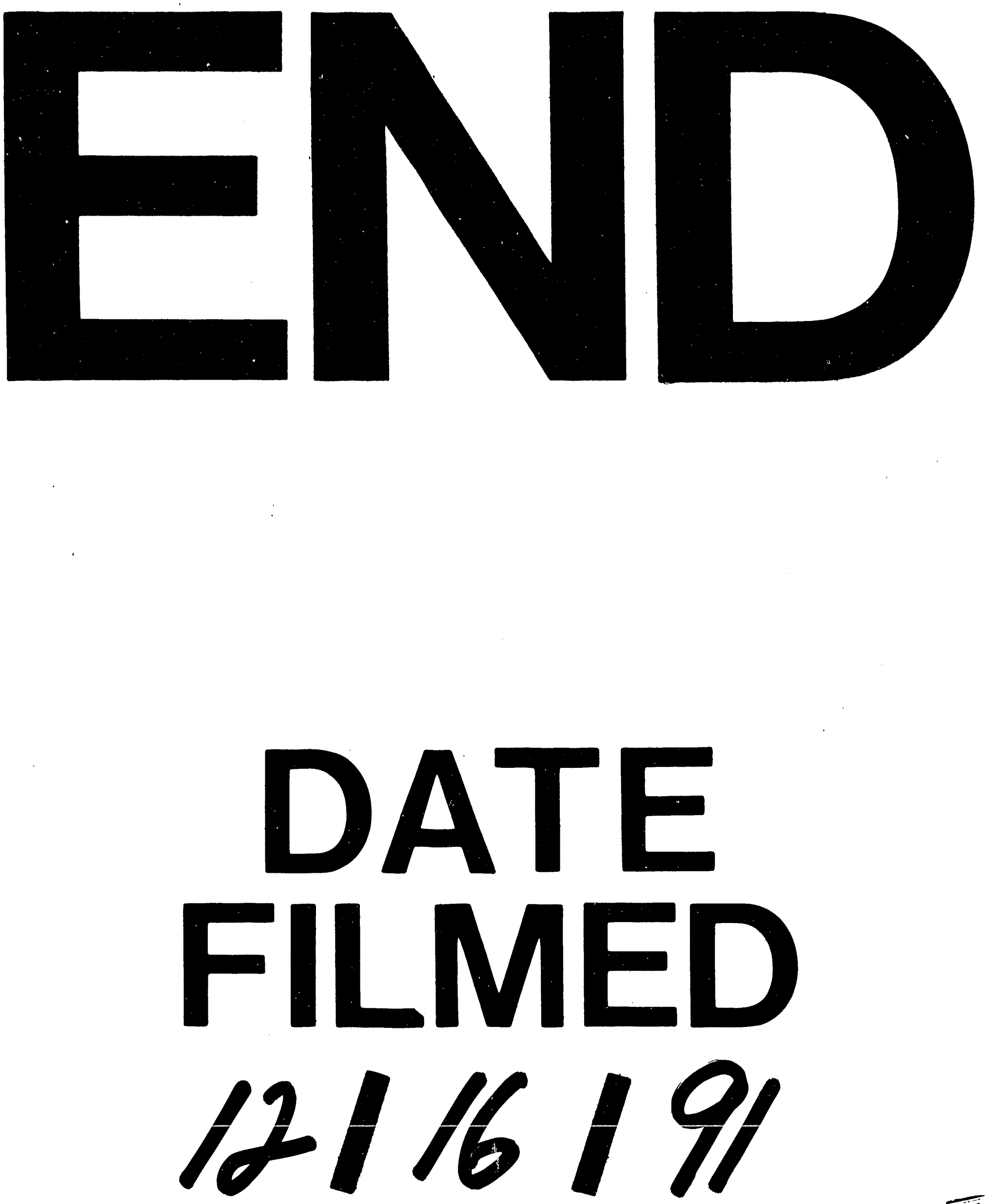

1 
